Carmine Anzivino

On the effect of capillarity and polarity in colloidal suspensions of nanoparticles
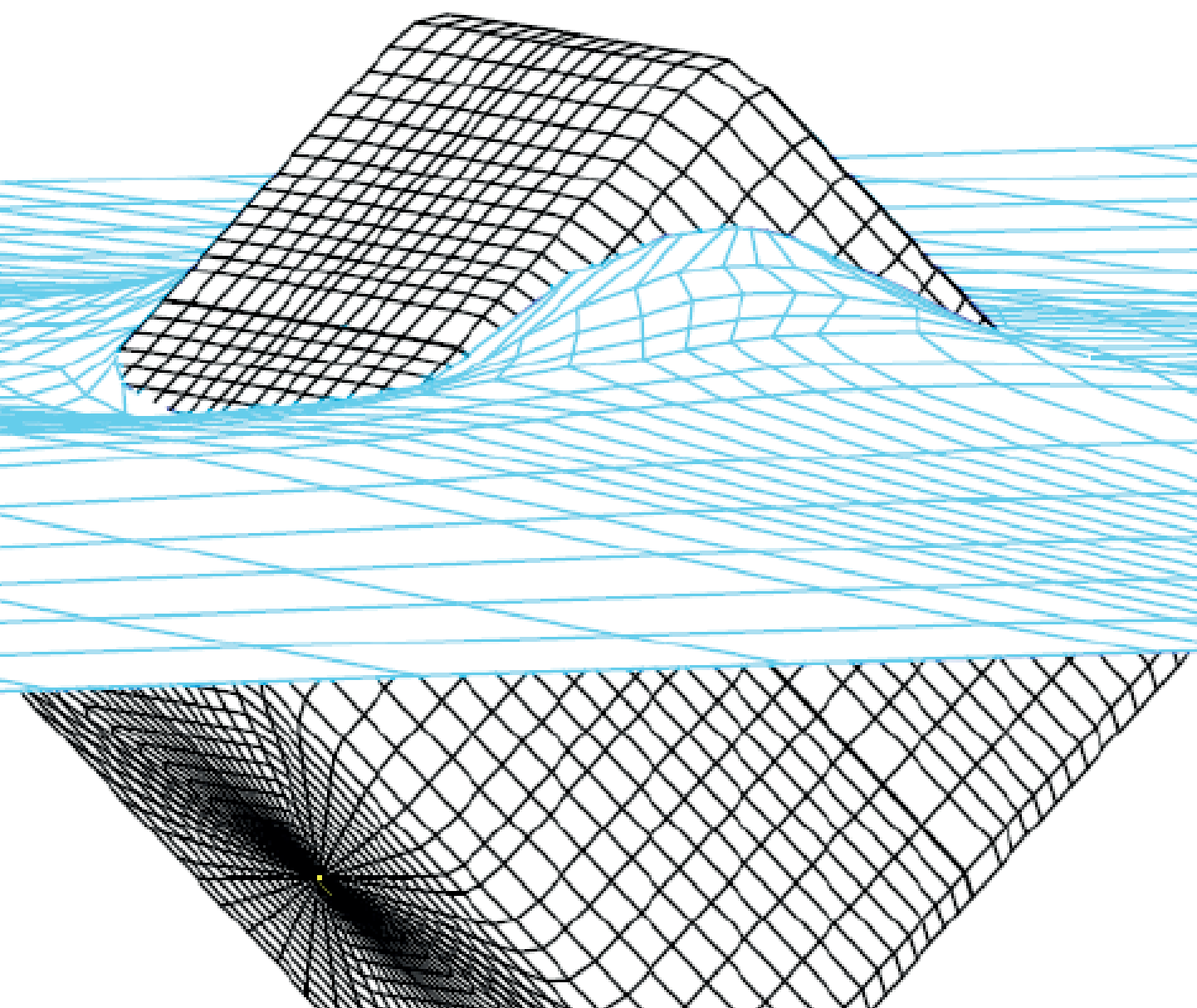



\section{On the effect of capillarity and polarity in colloidal suspensions of nanoparticles}

Carmine Anzivino 
Cover: Front page: sketch of a colloidal Janus dumbbell and a colloidal cube at a fluid-fluid interface. Back page: sketch of the twist-bend and splay-bend nematic phases stabilizing in systems of bent-core rods.

PhD thesis, Utrecht University, The Netherlands, May 2021

ISBN: 978-94-6423-265-3

Printed by ProefschriftMaken || www.proefschriftmaken.nl

A digital version of this thesis is available at the Utrecht University library repository https://dspace.library.uu.nl/

The work described in thesis was performed in the Soft Condensed Matter and Biophysics group, part of the Debye Institute for Nanomaterials Science at Utrecht University. 


\title{
On the effect of capillarity and polarity in colloidal suspensions of nanoparticles
}

\author{
Het effect van capillariteit en polariteit in colloïdale \\ suspensies van nanodeeltjes \\ (met een samenvatting in het Nederlands)
}

\section{Proefschrift}

ter verkrijging van de graad van doctor aan de Universiteit Utrecht

op gezag van de rector magnificus, prof. dr. H.R.B.M. Kummeling, ingevolge het besluit van het college voor promoties in het openbaar te verdedigen op maandag 14 juni 2021 des ochtends te 10:15 uur

door

\section{Carmine Anzivino}

geboren op 11 december 1990 te Foggia, Italië 
Promotor: $\quad$ Prof. dr. ir. M. Dijkstra

Prof. dr. R. H. H. G. van Roij

Assessment committee: $\quad$ Prof. dr. B. M. Mulder (AMOLF / Utrecht University) Prof. dr. P. P. A. M. van der Schoot (TU Eindhoven)

Dr. L. Tran (Utrecht University)

Prof. dr. R. A. Duine (Utrecht University)

Prof. dr. A. P. Mosk (Utrecht University)

This work is supported by the "Nederlandse Organisatie voor Wetenschappelijk Onderzoek" (NWO) TOPPUNT. This work was part of the D-ITP consortium, a program of the NWO that is funded by the Dutch Ministry of Education, Culture and Science (OCW). 


\section{Contents}

1 Introduction $\quad 1$

1.1 Phenomenological background . . . . . . . . . . . . . . . . 2

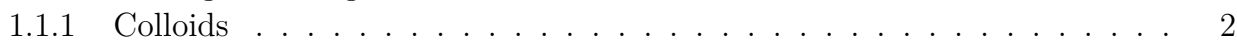

1.1.2 Chains of colloids at fluid-fluid interfaces . . . . . . . . . . . . . . 2

1.1.3 Liquid crystalline phases of polar particles . . . . . . . . . . . . . . 4

1.2 The equilibrium shape of fluid-fluid interfaces . . . . . . . . . . . . . 5

1.2.1 Young-Laplace equation and Young's law . . . . . . . . . . . . . 6

1.2.2 Numerical method . . . . . . . . . . . . . . . . . . . . 7

1.3 Phenomenological theories of liquid crystals . . . . . . . . . . . . . . 7

1.3.1 Oseen-Frank energy . . . . . . . . . . . . . . . . . . 8

1.3.2 Landau-de Gennes theory for uniaxial nematics . . . . . . . . . . . . 8

1.3.3 Flexoelectricity . . . . . . . . . . . . . . . . . . . . . . . . . . . . . . . 11

1.4 Scope and outline of this thesis f . . . . . . . . . . . . . . . 12

2 Equilibrium Configurations and Capillary Interactions of Janus Dumbbells and Spherocylinders at Fluid-Fluid Interfaces $\quad 15$

2.1 Introduction . . . . . . . . . . . . . . . . . . . . . . . . . . . . . . . . . . . .

2.2 Numerical method . . . . . . . . . . . . . . . . . . . . . . 17

2.3 Numerical Results . . . . . . . . . . . . . . . . . . . . . . . . 18

2.3.1 Single adsorbed particle . . . . . . . . . . . . . . . . . 19

2.3.2 Fit of the interfacial deformation field . . . . . . . . . . . . . . . . 23

2.3.3 Capillary pair interaction . . . . . . . . . . . . . . . . . . . . 24

2.4 Comparison wih experiments . . . . . . . . . . . . . . . . . . . . . . . . . . . . . . 25

2.4.1 Chains of dumbbells . . . . . . . . . . . . . . . . 25

2.4.2 Discussion and comparison with theoretical predictions . . . . . . . 28

2.5 Conclusions . . . . . . . . . . . . . . . . . . . . . . 29

2.6 Acknowledgments . . . . . . . . . . . . . . . . . . . 29

3 Chains of Cubic Colloids at Fluid-Fluid Interfaces 31

3.1 Introduction . . . . . . . . . . . . . . . . . . . . 32

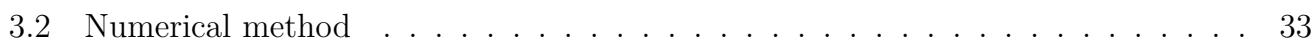

3.3 Results . . . . . . . . . . . . . . . . . . . . 34

3.3.1 Hexapolar and quadrupolar deformation fields . . . . . . . . . . . . . . 37

3.3.2 Stable chains of cubes at a fluid-fluid interface . . . . . . . . . . . . . 39

3.4 Conclusions and Discussion _. . . . . . . . . . . . . . . . . . . . . . . . . . . . . .

3.5 Acknowledgments . . . . . . . . . . . . . . . . . . . . . . . . . . . . . . . . . . . . . .

3.6 Appendix A: Periodic Boundary Conditions . . . . . . . . . . . . . . 48 
4 A Landau-de Gennes theory for twist-bend and splay-bend nematic phases $\begin{array}{ll}\text { of colloidal suspensions of bent rods } & 51\end{array}$

4.1 Introduction . . . . . . . . . . . . . . . . . . . . 52

4.2 Landau-de Gennes theory . . . . . . . . . . . . . . . . . . . 53

$4.3 \mathrm{I}-\mathrm{N}$ transition . . . . . . . . . . . . . . . . . . . . . 56

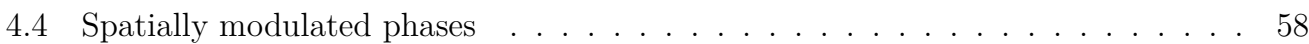

4.4.1 Comparison with the Oseen-Frank theory . . . . . . . . . . . . . . 58

4.4.2 Phase Transitions between spatially modulated nematic phases . . . . . . 61

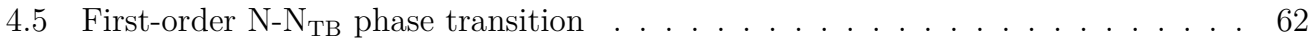

4.6 Renormalized elastic constants . . . . . . . . . . . . . . . . . . . . 67

4.7 Conclusions and discussion . . . . . . . . . . . . . . . . . . . . . . . . . . 69

4.8 Acknowledgements . . . . . . . . . . . . . . . . . . . . . . 69

4.9 Appendix A: Grand potential density of the $N_{\mathrm{TB}}$ and $N_{\mathrm{SB}}$ phases for $l_{3} \neq 0 . \quad$. 69

5 Evidence of density modulations in the splay-bend nematic phase of colloidal suspensions of bent rods

5.1 Introduction . . . . . . . . . . . . . . . . . . . . . . . . . 74

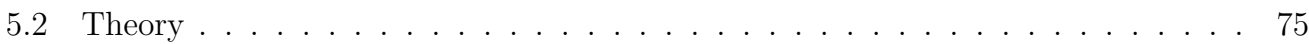

5.3 Results . . . . . . . . . . . . . . . . . . . . . 77

5.4 Conclusions and discussion . . . . . . . . . . . . . . . . . . 81

5.5 Appendix A: Derivation of Eq. (5.10) . . . . . . . . . . . . . . . . 81

$\begin{array}{lc}\text { References } & 83\end{array}$

$\begin{array}{lc}\text { Summary } & 93\end{array}$

$\begin{array}{ll}\text { Samenvatting } & 95\end{array}$

$\begin{array}{lr}\text { Acknowledgements } & 97\end{array}$

$\begin{array}{lr}\text { List of publications } & 99\end{array}$

$\begin{array}{ll}\text { Oral and poster presentations } & 100\end{array}$

$\begin{array}{ll}\text { About the author } & 101\end{array}$ 


\section{Introduction}

In this Chapter we introduce the reader to the main topics studied in this thesis. We start by presenting the phenomenological background that motivated our study. We proceed by briefly reviewing the main theoretical tools needed to deal with the introduced phenomenological background. Finally, we present an outline of the work performed in the subsequent chapters. 


\subsection{Phenomenological background}

\subsubsection{Colloids}

This thesis deals with a series of phenomena occurring in systems composed of particles with dimensions ranging from a few nanometers up to several microns. The structural properties of these so-called colloidal systems can easily be altered by mechanical or thermal stress of the order of thermal fluctuations. In this sense they belong to the broad branch of physics known as soft matter [1]. Examples of colloidal systems are present in tremendous numbers in every day life: suspensions/emulsions such as milk, blood, paint, liquid crystal displays in electronic equipment, gels and lotions, polymers, biological entities such as bacteria, sperm cells, tissues, etc.

A very important property of colloids is that they perform a random motion, when suspended in a solvent such as water or oil. This random motion is commonly referred to as Brownian motion, since observed for the first time by R. Brown in 1827 [2] for pollen particles. The theoretical explanation of Brown's observations was provided by A. Einstein in 1905 [3] amongst others, and, a few years later, definitive evidence of Brownian motion was provided in experiments by J. Perrin [4]. Because of the Brownian motion, colloidal systems are well suited to be studied by statistical mechanics. Indeed, colloids in a solvent are able to move around in the solvent and can interact with each other or with the solvent molecules. As a consequence they are in principle able to explore all possible configurations in which the system (colloids+solvent) can be, thus satisfying the ergodic hypothesis, which is the central assumption of statistical mechanics. However, as in atomic and molecular systems, ergodicity also breaks in colloidal systems when approaching the glass transition $[5,6]$.

On the experimental side, the possibility of manipulating colloidal systems has widely increased in the last decades. Indeed, important advancements have been made in preparing colloidal suspensions and tuning their properties [7,8]. Moreover, because of their size and slow dynamics, colloids are well suited to be studied in real space and real time using advanced microscopy techniques [9].

Out of the endless possibilities of colloidal systems, we will focus in this thesis on colloids trapped at a fluid-fluid interface, and on liquid crystalline colloidal systems.

\subsubsection{Chains of colloids at fluid-fluid interfaces}

A situation in which colloidal particles display striking features is when they are trapped at a fluid-fluid interface, i.e. at the interface of two immiscible fluids. Indeed, as firstly experimentally observed at the beginning of the twentieth century [10,11], and successively theoretically shown in a seminal paper by Pieranski [12], sub-millimiter sized particles strongly adsorb at fluid-fluid interfaces in order to reduce the interfacial area, and hence the free energy of the interface [13]. Once adsorbed, colloidal particles can give rise to stable monolayers.

Recently, capillarity has been invoked to explain this intriguing phenomenon [14]. Indeed, nonspherical colloidal particles adsorbed at a fluid-fluid interface induce interfacial deformations that determine their orientations and generate mutual capillary interactions which drive the particles to assemble into 2D ordered structures.

Very interestingly, it has been showed that this self-assembly process may be regulated by controlling the size, the shape, and the surface chemistry of the adsorbed particles [15]. As a consequence, a route can be established to build quasi-2D ordered structures at interfaces, 

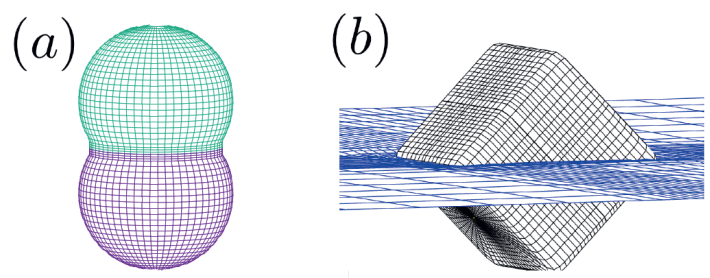

Figure 1.1: (a) Sketch of a Janus dumbbell consisting of two "faces". We use two colours to indicate that the two "faces" have different chemical surface properties. (b) Cubic particle adsorbed at a fluid-fluid interface with one side upwards with respect to the interface.

with applications ranging from the stabilization of foams and Pickering emulsions [16] to the development of new materials [17]. Because of the large variety of colloidal particles nowadays available, with tunable shapes and surface functionalizations $[8,18]$, the self-assembly of colloids trapped at a fluid-fluid interface has recently gained a great deal of attention in the softmatter community, and the behavior at interfaces of $\mathrm{nm}$ - and $\mu \mathrm{m}$-sized particles with several anisotropic shapes, e.g. rods, cylinders, cubes, dumbbells and discs [19-25], as well as particles with chemical heterogeneities on the surface, i.e. patchy and Janus particles [26-28], has been widely studied in the past few years.

In this thesis we mainly focus on chainlike aggregates formed by colloidal particles trapped at a fluid-fluid interface. The phase behaviour of colloidal Janus dumbbells, i.e. dumbbells with two "faces" having different chemical surface properties (see Fig. 1.1 $(a)$ ), adsorbed at a water-decane interface has been recently experimentally studied $[29,30]$. The observed scenario is puzzling. After the initial formation of an ordered crystalline monolayer, the dumbbells start to attach laterally, giving rise to chains of laterally aligned dumbbells. Very interestingly, if the dumbbells consist of two symmetric spheres, the resulting chains have a straight shape, while, if the dumbbells consist of two slightly asymmetric spheres, the resulting chains have a curved shape. Moreover, the dumbbells only attach laterally, and (almost) never by connecting their "heads". Several theoretical questions arise. "Is capillarity able to explain this phase behaviour?" "Since the spheres that make up the dumbbells have an almost isotropic shape, is the Janus nature of these particles the cause of interfacial deformations?" "Which kind of deformation field could explain the stabilization of (straight or curved) chains rather than 2D structures?"

Chainlike structures at interfaces have been recently experimentally observed also in the case of polystyrene $\mu \mathrm{m}$ sized cubes, adsorbed at a water-air interface [31,32]. In these chains the cubes are adsorbed with one side pointing upward with respect to the fluid-fluid interface (see Fig. 1.1(b)), and are all laterally attached. This experimental finding is surprising. Indeed, previous theoretical calculations $[33,34]$ have showed that (hydrophilic) colloidal cubes prefer to adsorb at a fluid-fluid interface with one vertex pointing upward with respect to the interface plane, and induce a hexapolar deformation field in the interface height profile which causes nmsized cubes to self-assemble into thermodynamically stable honeycomb, square and hexagonal lattices. Moreover, colloidal cubes adsorbed with one side up have been predicted not to induce any interfacial deformation, such that capillarity does not appear to play any role in this case.

We will deal with these phenomena and explain them, in the first part of the thesis. 


\subsubsection{Liquid crystalline phases of polar particles}

Liquid crystals are phases of matter sharing properties of both liquids and crystals, e.g. they can flow as liquids while they scatter light as crystals, typically found in suspensions of non-spherical particles. The simplest example is that of rod-like objects such as cholesterol molecules and Tobacco Mosaic Virus (TMV) particles [35]. At high temperatures or at low (osmotic) pressures, these systems typically show a stable isotropic $(I)$ fluid phase (see Fig. 1.2 $(a)$ ), characterized by the absence of any positional and orientational order. By contrast, when cooled or compressed, the simplest of the liquid crystalline phases, known as the nematic $(N)$ phase (see Fig. 1.2(b)), stabilizes. In the $N$ phase, while centers of mass of the particles are homogeneously distributed in space, the long axes of the particles align on average along a preferred direction $\hat{\mathbf{n}}$, such that the system exhibits orientational order in the absence of translational order.

The first attempt to explain on a microscopic ground the formation of the $N$ phase in systems of rod-like particles, was realized by Onsager in a seminal paper published in 1949 [36]. Onsager demonstrated that the orientational symmetry of a fluid of Brownian rod-like particles can break as a consequence of the anisotropic shape, at sufficiently high particle concentration. Since then, numerous liquid crystal phases displaying increasing complexity have been discovered or predicted in systems of particles with different shapes and symmetries. These include smectic phases [37-39], in which rod-like particles form layers, columnar phases [40,41], in which rodlike or disc-like particles form columns, cholesteric phases [42-44], in which particles display a helical arrangement, and biaxial nematic phases [45-48], in which particles can align along two preferred directions.

The importance of particle shape in determining the phase behavior of liquid crystals has nowadays been generally recognized, even if the mechanism connecting the macroscopic structure to the microscopic details of the constituent particles is still an open problem in many cases.

A great deal of attention has been recently attracted by systems of polar particles, having a "banana-like" or bent-core shape that favor spontaneous bend deformations in the nematic director field [49]. By noting that a pure bend deformation cannot uniformly fill the 3D space, Meyer [50] and Dozov [51] suggested that the local bend deformations have to be accompanied by either a twist or splay deformation. In the former case they theoretically postulated the stabilization of a spatially modulated twist-bend nematic $\left(N_{\mathrm{TB}}\right)$ phase, in the latter the stabilization of a spatially modulated splay-bend nematic $\left(N_{\mathrm{SB}}\right)$ phase was predicted. While the $N_{\text {TB }}$ phase displays a heliconical variation with bend and twist deformation in the molecular orientation (see Fig. 1.2(c)), the $N_{\mathrm{SB}}$ phase is characterized by alternating domains of splay and bend (see Fig. 1.2(d)). On the basis of these theoretical notions, an intriguing analogy between chirality and polarity has been suggested to hold in liquid crystals $[52,53]$. Just as chirality induces twist deformations, polarity induces bend deformations in the nematic director field of bent-core liquid crystals. This analogy has been further strenghtened by the prediction of a polar analog of the chiral blue phases [54]. Nevertheless, still more work is needed to confirm this extremely intriguing picture. Indeed, several alternative theoretical models have been proposed that do not involve polarity to explain the onset of spatially modulated nematic phases in bent-core liquid crystals phase [51,55-60].

While most of the research on bent-core liquid crystals has focused on thermotropic bent-core mesogens that become liquid crystalline upon lowering the temperature, very recently various routes have been developed to synthesize lyotropic colloidal model systems of bent particles, e.g. colloidal silica rods with a sharp kink or smoothly curved SU-8 rods [61-63]. By contrast 
(a)

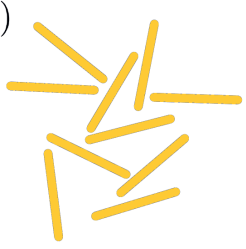

(b)

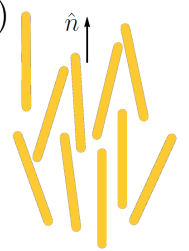

(c)

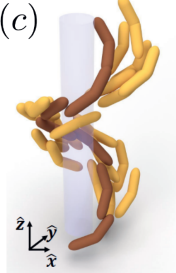

$(d)$

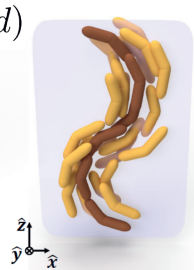

Figure 1.2: (a) Isotropic fluid phase of hard rods, characterized by the absence of any positional and orientational order. (b) Nematic phase of hard rods, characterized by orientational order in the absence of positional order. (c) Twist-bend nematic phase of hard bent rods, characterized by a heliconical variation of the particle orientation along the z-axis. (d) Splay-bend nematic phase of hard bent rods, characterized by alternating domains of splay and bend in the $x$ - $z$ plane.

with thermotropic systems, the liquid crystalline behavior of these colloidal systems is driven by concentration. For lyotropic systems, several predictions have been formulated by means of simulations and microscopic theories. By using Onsager theory [64], a first-order uniaxial $N$ to $N_{\text {TB }}$ phase transition has been found in a system of hard curved particles at sufficiently high particle concentrations. Computer sumulations on systems of hard bent spherocylinders [65] have not only confirmed this $N-N_{\text {TB }}$ phase transition, but have also predicted the existence of a subsequent $N_{\mathrm{TB}}-N_{\mathrm{SB}}$ phase transition. A phenomenological theory able to relate these findings to the polar nature of bend particles has not yet been introduced for lyotropic systems.

Another open question in the field of bent-core liquid crystals concerns the existence and the true nature of the $N_{\mathrm{SB}}$ phase. By contrast with the $N_{\mathrm{TB}}$ phase, an $N_{\mathrm{SB}}$ phase has never been found in thermotropic systems of bent-core molecules. Inspired by the simulation results of Ref. [65], an $N_{\mathrm{SB}}$ phase has instead been recently observed in lyotropic systems of colloidal smoothly curved SU-8 rods [66], and of polydisperse bent colloidal silica rods [67]. However, the $N_{\mathrm{SB}}$ phase found in simulations of hard curved rods also displays density modulations. The question arises: "is the $N_{\mathrm{SB}}$ phase actually a smectic phase?"

We will deal with the phase behavior of lyotropic suspensions of colloidal bent rods in the second part of this thesis.

\subsection{The equilibrium shape of fluid-fluid interfaces}

In order to investigate the self-assembly of colloidal particles adsorbed at a fluid-fluid interface it is important to know how to deal with the behavior of a fluid-fluid interface in contact with a solid surface. This is the main aim of the following Section. In our discussion we will neglect gravitational effects, as these do not play a role in the case of colloidal particles adsorbed at a fluid-fluid interface. To understand this point we introduce the so-called Bond number Bo $\equiv(L / \sqrt{\gamma /(g \delta)})^{2}$, where $\delta$ is the mass density difference between the particle and the fluids, $\gamma$ is the fluid-fluid surface tension, $g$ is the gravitational acceleration on planet Earth, and $L$ is a characteristic length of the system (e.g. the radius in case of spherical particles or the side in the case of cubic particles). In physical systems with $\mathrm{Bo} \ll 1$ gravitational effects can be neglected, while in physical systems with Bo $\gg 1$ gravitational effects must be taken into account. By considering typical values $\delta \sim 10^{3} \mathrm{~kg} \mathrm{~m}^{-3}$ and $\gamma \sim 10^{-2} \mathrm{~N} \mathrm{~m}^{-1}$, it follows that 


\section{Fluid 1}

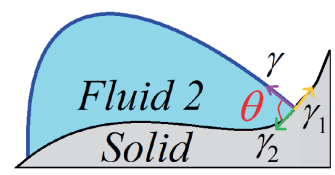

Figure 1.3: A fluid-fluid interface in contact with a solid surface forms a contact angle $\theta$ at the three-phase contact point. $\theta$ is related to the fluid-fluid surface tension $\gamma$, the fluid 1-solid surface tension $\gamma_{1}$ and the fluid 2-solid surface tension $\gamma_{2}$ by the Young law (Eq. (1.2)). Together with the Young-Laplace equation (Eq. (1.1)), the Young law gives the equilibrium shape of the fluid-fluid interface.

Bo $\ll 1$ for colloidal particles with $L \ll 1 \mathrm{~mm}$.

\subsubsection{Young-Laplace equation and Young's law}

Let us consider two immiscible, homogeneous, and incompressible molecular fluids (say fluid 1 and fluid 2) in the presence of an external solid surface (see Fig. 1.3). The two demixed fluids are separated by a fluid-fluid interface. To this interface, an energy cost $\gamma S$ is assigned, where $S$ is the surface area of the interface, and $\gamma$ is the associated surface tension. The preferred shape of the interface is the one with minimum (free) energy, i.e., minimum $S$. This correspond to a flat surface for a free meniscus. However factors like the wettability of the solids in contact with the interface, electrostatic fields, etc., influence the energy of the interface that includes a particle and so deform it in a non-trivial way. In absence of electric fields, the fluid-fluid interface shape is described by the Young-Laplace equation and Young's law [13]. In order to introduce the Young-Laplace equation and Young's law, we assume that the "height" of the fluid-fluid interface at lateral position $(x, y)$ can be written as a function $h(x, y)$, where we have introduced a Cartesian coordinate system with the $z$ axis anti-parallel to the gravity direction. When gravitational effects can be neglected, the Young-Laplace equation reads

$$
\nabla \cdot \hat{\mathbf{n}}(x, y)=\frac{\Delta P}{\gamma}
$$

where $\nabla \equiv\left(\frac{\partial}{\partial_{x}}, \frac{\partial}{\partial_{y}}, \frac{\partial}{\partial z}\right), \hat{\mathbf{n}}(x, y) \equiv \nabla(z-h(x, y)) /|\nabla(z-h(x, y))|$ is the unit normal to the surface $h(x, y)$ in the point $(x, y, h(x, y))$, and $\Delta P \equiv P_{2}-P_{1}$ is the difference between the bulk pressures $P_{1}$ and $P_{2}$ of the two fluids, on either sides of the interface. The Young law reads

$$
\cos \theta=\frac{\gamma_{1}-\gamma_{2}}{\gamma}
$$

where $\gamma, \gamma_{1}$ and $\gamma_{2}$ are the fluid-fluid, fluid 1-solid and fluid 2-solid surface tensions, respectively (see Fig. 1.3).

For the scope of this thesis it is convenient to observe that, as shown in Ref. [68], solving the Young-Laplace equation (1.1) and the Young law (1.2), is equivalent to minimizing the thermodynamic grand potential $\Omega_{t}[h]$ of a fluid-fluid system in contact with a solid, with respect to the fluid-fluid interface shape $h(x, y)$, i.e. to solving the Euler-Lagrange equation

$$
\frac{\delta \Omega_{t}[h]}{\delta h(x, y)}=0 .
$$


In general $\Omega_{t}[h]$ takes the form

$$
\Omega_{t}[h]=\gamma S[h]+\left(\gamma_{2}-\gamma_{1}\right) W_{2}[h]-P_{1} V_{1}[h]-P_{2} V_{2}[h],
$$

where $S$ is the area of the fluid-fluid interface, $W_{2}$ is the area of the solid surface in contact with fluid 2, and $V_{1}$ and $V_{2}$ are the volumes of fluid 1 and fluid 2, respectively. We observe that in Eq. (1.4), $P_{1}$ and $P_{2}$ act as Lagrange multipliers that depend on the choice of $V_{1}$ and $V_{2}$.

\subsubsection{Numerical method}

The Young and Young-Laplace equations have an analytic solution only in the simplest cases [69]. Most commonly they have to be solved numerically. In this thesis we employ the numerical method introduced in Ref. [68], which is based on a minimization of the thermodynamic potential (1.4). More specifically we find the equilibrium shape $h(x, y)$ of a fluid-fluid interface for a given volume of each fluid, by calculating numerically the interface shape that minimizes the following thermodynamic-potential functional:

$$
E[h]=\gamma S[h]+\left(\gamma_{2}-\gamma_{1}\right) W_{2}[h],
$$

with the constraint that the volumes $V_{1}$ and $V_{2}$ of fluid 1 and fluid 2 have a preset value. We note that this procedure is equivalent to solving the Euler-Lagrange equation (1.3).

To minimize $E[h]$ we treat $h(x, y)$ as a grid of points. We apply a simulated annealing algorithm [70], which consists of an adaptation of a Monte Carlo method, where a temperature is artificially introduced and gradually lowered during the simulation. We change randomly the point positions, keeping the fluid volumes $V_{1}$ and $V_{2}$ constant and rejecting unphysical (e.g. overlapping) configurations. Every configuration change is then accepted with probability

$$
P \equiv \begin{cases}1, & \text { if } \Delta E<0 \\ \exp \left(-\frac{\Delta E}{k_{B} T}\right), & \text { if } \Delta E \geq 0\end{cases}
$$

where $\Delta E$ is the change of (free) energy $E$ between the old and the new configuration of points, $T$ is the temperature-like control parameter, and $k_{\mathrm{B}}$ is the Boltzmann constant. In a simulation, these configuration changes are performed continuously while $T$ is gradually lowered starting from an initial $T_{0}$. When $T$ approaches zero, the simulation ends.

\subsection{Phenomenological theories of liquid crystals}

Several theoretical frameworks have been introduced to deal with the liquid-crystalline phases of matter. These can be roughly divided into microscopic and phenomenological approaches. While in the former the discrete nature of the constituent particles is explicitly taken into account, in the latter it is not. Examples of microscopic approaches range from Onsager's theory [36] and classical density functional theory [71], to particle-based computer simulations $[72,73]$. The two principal phenomenological approaches of liquid crystals are the Oseen-Frank theory for the director field [74,75], and the Landau-de Gennes theory based on the Q-tensor order parameter [76]. In this thesis phenomenological approaches will be used to describe the liquid crystalline order. We review them in the following Section. We also review the so-called flexoelectric effect which is of crucial importance for this thesis. 


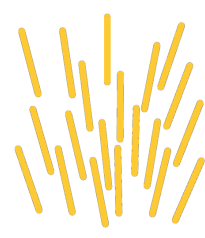

Splay

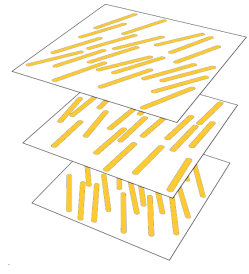

Twist

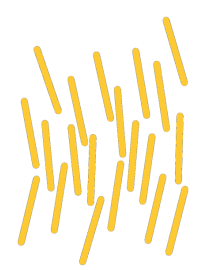

Bend

Figure 1.4: Sketch of the three independent elastic deformations of nematic liquid crystals: splay, twist, and bend.

\subsubsection{Oseen-Frank energy}

In nematic liquid crystals, the long axes of the particles align on average along a preferred direction. This direction is identified by a so-called nematic director $\hat{\mathbf{n}}(\mathbf{r}) \equiv \hat{\mathbf{n}}$, satisfying the relations $|\hat{\mathbf{n}}|^{2}=1$. By contrast with systems of magnetic particles [77], in nematic liquid crystals the up-down symmetry holds, such that $\hat{\mathbf{n}}$ and $-\hat{\mathbf{n}}$ are equivalent. While the preferred state of a nematic liquid crystal is one of uniform alignment along $\hat{\mathbf{n}}$, distortions of the nematic director can also occur in many cases. In a nematic liquid crystal, three main independent distortions of $\hat{\mathbf{n}}$ exist, which are known as splay, twist and bend, respectively, and are sketched in Fig. 1.4. In terms of derivatives of $\hat{\mathbf{n}}$ they correspond to the following expressions being non-zero:

$$
\begin{array}{ll}
\nabla \cdot \hat{\mathbf{n}} & \text { Splay } \\
\hat{\mathbf{n}} \cdot \nabla \times \hat{\mathbf{n}} & \text { Twist } \\
\hat{\mathbf{n}} \times(\nabla \times \hat{\mathbf{n}}) & \text { Bend } .
\end{array}
$$

On the basis of these considerations, nematic liquid crystals can be characterized by a so-called Oseen-Frank elastic energy

$$
F=\frac{1}{2} K_{11}(\nabla \cdot \hat{\mathbf{n}})^{2}+\frac{1}{2} K_{22}\left(\hat{\mathbf{n}} \cdot \nabla \times \hat{\mathbf{n}}+q_{0}\right)^{2}+\frac{1}{2} K_{33}(\hat{\mathbf{n}} \times(\nabla \times \hat{\mathbf{n}}))^{2},
$$

where $K_{11}, K_{22}$, and $K_{33}$ are known as the splay, twist and bend elastic constants, respectively, often also called Oseen-Frank elastic constants. The wave number $q_{0}$ is non-zero only in chiral liquid crystals. In such chiral systems this coefficient serves indeed to favour a naturally distorted texture in which the director displays a pure twist equilibrium distortion with $\hat{\mathbf{n}} \cdot \nabla \times \hat{\mathbf{n}}=-q_{0}[78]$.

\subsubsection{Landau-de Gennes theory for uniaxial nematics}

The characterization of liquid crystals in the Oseen-Frank theory is performed on a purely elastic ground, by considering the distortions of the nematic director field $\hat{\mathbf{n}}$. Nevertheless, to properly characterize the degree of order displayed by liquid crystals, besides $\hat{\mathbf{n}}$, the distribution of the particle orientations must be taken into account. Let us call $P(\hat{\mathbf{u}}) d \hat{\mathbf{u}}$ the probability that a particle has an orientation on the surface element $d \hat{\mathbf{u}}$ centered about $\hat{\mathbf{u}}$ on the unit sphere. Because nematics obey cylindrical symmetry, if we consider a coordinate system in which $\hat{\mathbf{n}}$ is along the $z$-axis (see Fig. $1.5(a)$ ), the probability density can be written as $P(\hat{\mathbf{u}})=P(\hat{\mathbf{u}} \cdot \hat{\mathbf{n}})=$ 

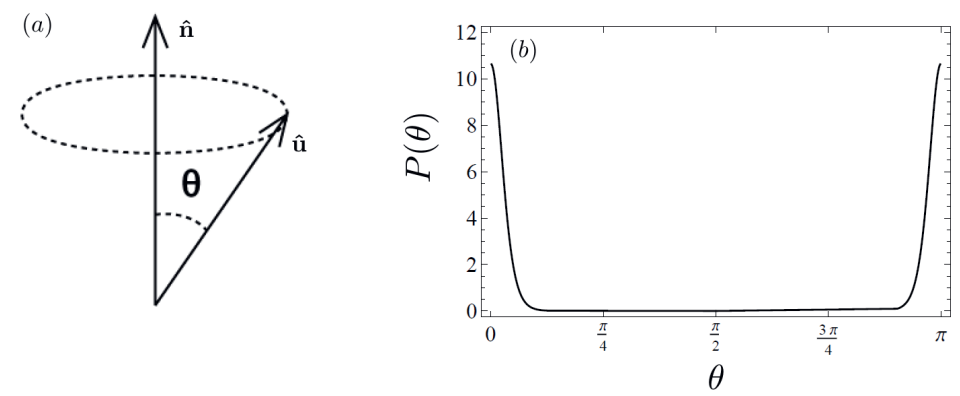

Figure 1.5: $(a)$ The orientation $\hat{\mathbf{u}}$ of a single particle forms an angle $\theta$ with the nematic director field

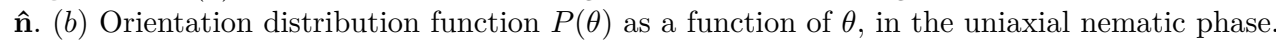
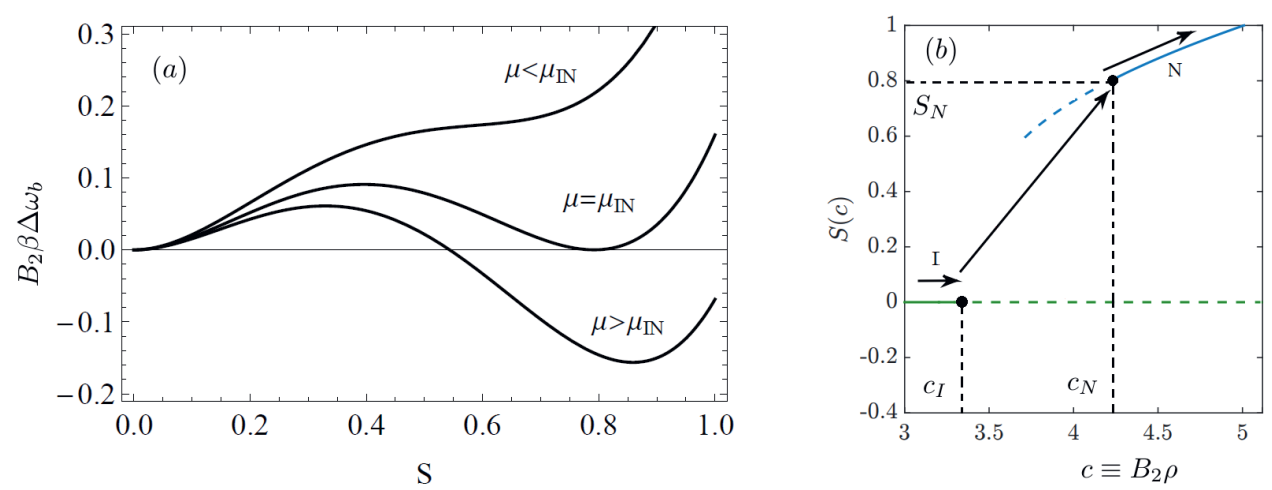

Figure 1.6: $(a)$ (Bulk) grand potential density $\Delta \omega_{b}$ as a function of the nematic order parameter $S$. A first-order $I-N$ phase transition occurs at $\mu_{I N}$. (b) Nematic order parameter $S$ as a function of the dimensionless particle concentration $c \equiv B_{2} \rho$. The $I-N$ transition is accompagnied by a jump in $S$ as well as in $c$. $B_{2}=\pi L^{2} D / 4$ is the second virial coefficient in the isotropic fluid phase of hard rods of lenght $L$ and diameter $D$, in the limit $L \gg D$. 
$P(\theta)$, where $\theta=\arccos (\hat{\mathbf{u}} \cdot \hat{\mathbf{n}})$ is the polar angle between $\hat{\mathbf{u}}$ and $\hat{\mathbf{n}}$. In the isotropic phase, $P(\theta)=1 /(4 \pi)$. An example of $P(\theta)$ in the nematic phase is given in Fig. 1.5 $(b)$. For our discussion it turns out useful to consider not the orientation distribution function $P(\theta)$ but rather one of its moments. While the first moment is zero by up-down symmetry, the second one is nonzero and is known as the nematic order parameter $S$, defined as

$$
S \equiv\left\langle\frac{3}{2}(\hat{\mathbf{u}} \cdot \hat{\mathbf{n}})^{2}-\frac{1}{2}\right\rangle=\left\langle\frac{3}{2} \cos ^{2} \theta-\frac{1}{2}\right\rangle=\left\langle P_{2}(\cos \theta)\right\rangle,
$$

where $\langle\ldots\rangle=\int_{-1}^{1} d \cos \theta \int_{0}^{2 \pi} d \phi(\ldots) P(\theta)$ is an orientational average and $P_{2}(x)=\left(3 x^{2}-1\right) / 2$ is the second Legendre polynomial. The nematic order parameter $S$ quantifies the fluctuations of the particle orientations around $\hat{\mathbf{n}}$. For perfectly aligned particles we have $S=1$, while for randomly oriented particles we have $S=0$.

The director $\hat{\mathbf{n}}$ and the nematic order parameter $S$ are usually joined into a single tensorial order parameter. This is the second-rank, traceless tensor [76]

$$
\mathbf{Q}=\frac{S}{2}(3 \hat{\mathbf{n}} \otimes \hat{\mathbf{n}}-\mathbf{1}),
$$

that, from a physical point of view may be thought of as proportional to the anisotropic part of the magnetic susceptibility, or of the dielectric tensor. The largest eigenvalue of $\mathbf{Q}$ is the scalar nematic order parameter $S$, and the corresponding eigenvector is the director $\hat{\mathbf{n}}$. The other two eigenvalues are equal to $-S / 2$ for uniaxial nematics, and the corresponding eigenvectors are perpendicular to $\hat{\mathbf{n}}$.

The tensorial order parameter (1.10) can be exploited to build a phenomenological Landau theory for nematic liquid crystals. Since introduced by de Gennes in 1971 [79], this theory is nowadays known as the Landau-de Gennes theory. It is based on the hypothesis that equilibrium properties of nematic liquid crystals can be found from a generalized free energy, constructed from frame invariant-contractions of $Q_{\alpha \beta}$ and spatial derivatives $\partial_{\lambda} Q_{\alpha \beta}$. A restriction on the expansion is that it must be stable against an unlimited growth of $\mathbf{Q}$.

In the original work of de Gennes, the temperature-dependent Helmholtz (or Gibbs) free energy $F(T)$ was expanded in powers of $\mathbf{Q}$. While this expansion is suitable for dealing with thermotropic systems, i.e. systems which become ordered as a function of temperature, it fails in properly describing lyotropic systems, i.e. systems which become ordered as a function of density. In this thesis, however, we are mainly interested in lyotropic systems. As recently shown in Ref. [80], to deal with lyotropic systems it is convenient to set up a Landau expansion of the chemical-potential dependent grand potential $\Omega$ rather than of the temperature-dependent Helmholtz (or Gibbs) free energy $F$. By using $\Omega$, the density dependence will naturally be encoded through the relation $\partial(\Omega / V) /\left.\partial \mu\right|_{V, T}=-\rho$, with $V$ the volume of the system and $\rho$ the average density. In particular, in Ref. [80] it has been showed that by expanding $\Omega$ in powers of Q, a proper characterization of the $I-N$ transition displayed by hard rods can be obtained. To show this we consider a system of hard rods of contour length $L$ and diameter $D$, at chemical potential $\mu$ in a macroscopic volume $V$ at a fixed temperature $T$. We write the LdG grand potential

$$
\Delta \Omega(\mathbf{Q})=\int_{V} d \mathbf{r}\left[\Delta \omega_{b}(\mathbf{Q})+\omega_{e}(\mathbf{Q}, \nabla \mathbf{Q})\right]
$$

where $\Delta \omega_{b} \equiv \Delta \omega_{b}(\mathbf{Q} ; \mu)$ is the excess bulk grand potential density with respect to the isotropic state, and $\omega_{e} \equiv \omega_{e}(\mathbf{Q}, \nabla \mathbf{Q})$ describes the elastic deformations. We expand $\Delta \omega_{b}$ and $\omega_{e}$ in units 
(a)

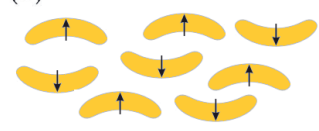

(c)

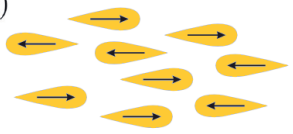

(b)

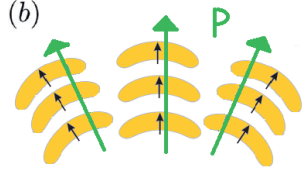

$(d)$

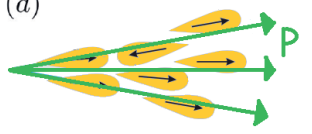

Figure 1.7: Unpolarised "banana-shaped" particles (a) may spontaneously polarise $(b)$ upon a bend deformation, or a bend deformation may lead to polarisation. Analogously, unpolarised "pear-shaped" particles $(c)$ may spontaneously polarise $(d)$ upon a splay deformation, or a splay deformation may lead to polarisation.

of $\beta^{-1}=k_{B} T$ with $k_{B}$ the Boltzmann constant, until fourth order in $\mathbf{Q}$ and second order in $\nabla \mathbf{Q}$, respectively, i.e. we write

$$
\beta B_{2} \Delta \omega_{b}(\mathbf{Q} ; \mu)=\frac{2}{3} a \beta\left(\mu^{*}-\mu\right) Q_{\alpha \beta} Q_{\beta \alpha}-\frac{4}{3} b Q_{\alpha \beta} Q_{\beta \lambda} Q_{\lambda \alpha}+\frac{4}{9} d Q_{\alpha \beta} Q_{\beta \alpha} Q_{\lambda \rho} Q_{\rho \lambda},
$$

and

$$
\beta B_{2} \omega_{e}(\mathbf{Q}, \nabla \mathbf{Q})=\frac{2}{9} l_{1}\left(\partial_{\alpha} Q_{\beta \lambda}\right)\left(\partial_{\alpha} Q_{\beta \lambda}\right)+\frac{2}{9} l_{2}\left(\partial_{\alpha} Q_{\alpha \lambda}\right)\left(\partial_{\beta} Q_{\beta \lambda}\right),
$$

where we use Einstein's summation convention for repeated indices throughout this thesis. The second virial coefficient in the isotropic fluid phase is given by $B_{2}=\pi L^{2} D / 4$ in the limit $L \gg D$ and is included in our definition to render the Landau coefficients $a, b$, and $d$ conveniently dimensionless.

As shown in Ref. [80] and as we will review in Chapter 4, the expansion (1.11) can describe a first-order $I-N$ transition at chemical potential $\mu_{I N}$. As shown in Fig. 1.6 $(a)$, for $\mu<\mu_{I N}$ the minimum of the grand potential is found at $S=0$, while for $\mu>\mu_{I N}$ the minimum is found at $S>0$. Furthermore, by fitting the Landau coefficients to known results of Onsager theory, the well known jump associated to the $I-N$ transition of hard rods can be reproduced within our Landau-de Gennes theory. As shown in Fig. 1.6(b), at the $I-N$ transition we find a jump from $\left(c_{I}, S_{I}=0\right)$ to $\left(c_{N}, S_{N} \neq 0\right)$, where $c_{I}$ and $c_{N}$ indicate the dimensionless particle concentration in the isotropic and nematic phases, respectively.

Finally, it is important to observe that the parameters $l_{1}$ and $l_{2}$ in (1.13) can be related to the Oseen-Frank elastic constants. By inserting the tensor order parameter (1.10) into (1.13), it follows that $\beta D K_{11}=\beta D K_{33}=4 S^{2}\left(2 l_{1}+l_{2}\right) /\left(\pi L^{2}\right)$ and $\beta D K_{22}=8 S^{2} l_{1} /\left(\pi L^{2}\right)$. The degeneracy between $K_{11}$ and $K_{33}$ can be removed [81] by adding one of the possible couplings between $\mathbf{Q}$ and $\nabla \mathbf{Q}$, e.g. $Q_{\alpha \beta}\left(\partial_{\gamma} Q_{\alpha \gamma}\right)\left(\partial_{\xi} Q_{\beta \xi}\right)$, in the grand potential expansion (1.11).

\subsubsection{Flexoelectricity}

A property of liquid crystals that is important to the scope of this thesis is known as flexoelectricity. This consists of a coupling between elastic deformations and electrostatic polarization which has been discovered by Meyer in 1969 [82] to occur in liquid-crystalline phases of matter. 
Meyer showed that, in general, a splay or bend deformation of the nematic director $\hat{\mathbf{n}}$ of a liquid crystal medium leads to a spontaneous (electrostatic) polarization, which can be observed as a macroscopic dipole moment of the liquid crystal. The polarization is usually quantified by a vector $\mathbf{P}$. Meyer also showed that, conversely, an applied electric field induces an electrostatic polarization $\mathbf{P}$, which leads to a combination of splay and bend distortions in $\hat{\mathbf{n}}$. The flexoelectric effect may occur in liquid crystals due to electrostatic polarization but may also arise due to particle shape in systems of "pear-shaped" or "banana-shaped" particles in the absence of an electric field. The flexoelectric effect occurring in systems of "banana-shaped" particles, consists of a coupling between polarity and bend deformations (see Figs. 1.7 $(a)$ and $1.7(b))$, and is hence referred to as the bend flexoelectric effect. By contrast, the flexoelectric effect occurring in systems of "pear-shaped" particles, consists of a coupling between polarity and splay deformations (see Figs. $1.7(c)$ and 1.7(d)), and is referred to as the splay flexoelectric effect.

Flexoelectricity can be involved in the Landau-de Gennes framework by including in the expansion (1.11) a term $\lambda P_{\alpha}\left(\partial_{\beta} Q_{\alpha \beta}\right)$, which couples the polarization vector $\mathbf{P}$ and the divergence of the tensor $\mathbf{Q}$ with an amplitude $\lambda$ that we will refer to as the flexoelectric coupling parameter.

\subsection{Scope and outline of this thesis}

This thesis is divided into two main parts. In the first part (Chapters 2 and 3) we employ the numerical method reviewed in Section 1.2 of this Introduction to investigate the role played by capillarity in the formation of chainlike structures of colloidal particles adsorbed at a fluid-fluid interface. In particular, in Chapter 2 we consider a variety of particles, that interpolate between dumbbells and spherocylinders, trapped at a fluid-fluid interface. We find that the overall shape of the induced deformation strongly depends on the chemical nature (Janus or non-Janus) of the particle surface. We indeed find that Janus particles induce a strong hexapolar deformation field while non-Janus particles of the same shape do not induce any interfacial deformation. Furthermore, the hexapolar deformation field induces capillary attractions for laterally aligned Janus dumbbells and repulsions for laterally anti-aligned ones. Our numerical findings explain the experimental observations of chainlike structures of Janus colloidal dumbbells at a waterdecane interface. In Chapter 3, instead, we focus on the chains formed by colloidal cubes, as observed in experiments reported in Refs. [31,32]. In agreement with Refs. [33,34], we find that hydrophilic cubes, with a small Young contact angle $\theta \sim 0$, give rise to hexagonal aggregates. However, we find that, by decreasing $\theta$, a regime exists in which a lattice of $N \rightarrow \infty$ adsorbed cubes assemble into stable chains formed by cubes adsorbed with one edge pointing upward with respect to the interface plane, in agreement with experiments. The formation of the chains is induced by a quadrupolar deformation field rather than a hexapolar one.

The second part of this thesis (Chapters 4 and 5) is devoted to the investigation of nematic phases that become stable in lyotropic colloidal suspensions of curved or bent rods. In Chapter 4, we extend the Landau-de Gennes theory introduced in Ref. [80] and quickly reviewed in Section 1.3.2 of this Introduction, from the case of straight rods to that of curved rods. To this aim we exploit the bend flexoelectric effect as suggested in recent papers $[53,54]$. In particular we develop a phenomenological Landau-de Gennes theory for lyotropic colloidal suspensions of bent rods using a $Q$-tensor expansion of the chemical-potential dependent grand potential and introduce a bend flexoelectric term, coupling the polarization and the divergence of the $Q$-tensor. We study the stability of $N$, twist-bend $N_{\mathrm{TB}}$, and splay-bend $N_{\mathrm{SB}}$ nematic phases 
of colloidal bent rods. We connect the predictions of our theory to recent simulation results. Finally, in Chapter 5, we further extend the model introduced in Chapter 4 to include the possibility of a spatial modulation of the scalar nematic order parameter $S$. We show that the spatial modulations in $S$ are accompanied by a one-dimensional density modulation in the splay-bend nematic phase of colloidals systems, such that this $N_{\mathrm{SB}}$ phase has actually the key characteristics of a smectic rather than a nematic phase. By contrast, we find that the twist-bend $N_{\text {TB }}$ phase has a homogeneous density and is hence a proper nematic phase. 



\section{Equilibrium Configurations and Capillary Interactions of Janus Dumbbells and Spherocylinders at Fluid-Fluid Interfaces}

We numerically investigate the adsorption of a variety of Janus particles (dumbbells, elongated dumbbells and spherocylinders) at a fluid-fluid interface by using a numerical method that takes into account the interfacial deformations. We first determine the equilibrium configuration of a single adsorbed particle, and we find that the overall shape of the induced deformation field has a strong hexapolar mode while non-Janus particles of the same shape do not induce any interfacial deformation. We then calculate the capillary interactions between two Janus spherocylinders adsorbed at an interface. The hexapolar deformation field induces capillary attractions for laterally aligned Janus spherocylinders and repulsions for laterally anti-aligned ones. Our results reproduce the experimentally observed behavior of micrometer-sized charged Janus dumbbells adsorbed at a water-decane interface. 


\section{$2.1 \quad$ Introduction}

Colloidal particles with dimensions between $1 \mathrm{~nm}$ and $1 \mu \mathrm{m}$ strongly adsorb at a fluid-fluid interface and can self-assemble into $2 \mathrm{D}$ ordered structures $[10,11,83]$. The adsorption of colloids is a well understood phenomenon: the interfacial area and hence the free energy of the interface can be reduced by the adsorption of particles at the interface [13]. By contrast, the selfassembly process is still not well understood even though a well-known, macroscopic analogue exists in everyday life when mm-cm sized breakfast cereals cluster in a bowl of milk. In the case of this so-called Cheerios effect [84] the gravitational force pulls the cereals down causing deformations in the fluid interface that are in turn responsible for capillary attractions among the cereals and consequently for the self-assembly process. As the gravitational effects can be neglected in the case of colloidal particles [85-87], the colloidal self-assembly at a fluidfluid interface arises from a more intricate phenomenon, although capillarity still plays an important role as we will see. The self-assembly of colloids trapped at a fluid-fluid interface can only be explained by forces that are sufficiently long-ranged and extend over distances many times the particle dimension [88]. Hence, the colloidal self-assembly cannot be explained by electrostatic and Van der Waals forces [89] as these are not sufficiently long-ranged in most solvents. However, interfacial deformations are long-ranged. The interfacial deformations arising by sub-mm particles are induced by particle properties such as the shape and the surface chemistry rather than by gravitational effects [90-97]. The behavior of particles with homogeneous chemical properties but anisotropic shape, e.g. rods, cylinders, cubes, dumbbells and discs, at fluid-fluid interfaces has been widely studied [19,20,25,98]. Since the combined effect of the anisotropic shape and the heterogeneity in the surface chemistry is emphasized in the case of so-called Janus particles, i.e. particles with two "faces" having different chemical surface properties $[1,99]$, many experimental investigations have been focused on their selfassembly at an interface [26-28, 100-107].

A pioneering study on the self-assembly of colloids at a fluid-fluid interface was performed by Pieranski [12]. In this work interfacial deformation effects were neglected and a flat interface was assumed. This assumption, often used in literature, is actually only valid in the case of homogeneous spherical particles in equilibrium. In all other cases, it is equivalent to ignoring the induced interfacial deformation field. Nevertheless, following Pieranski's assumption of a flat interface, several numerical techniques have been employed for calculating the equilibrium configuration of an arbitrarily shaped particle adsorbed at a fluid interface: e.g. the triangular tessellation technique (TTT) [108-113], and a hit and miss Monte Carlo method [26, 27, 100]. In order to take into account deformations of the fluid interface that arise when a particle is adsorbed, various numerical methods exist, like the widely used Surface Evolver program [114], or a recently introduced simulated annealing method [68]. By using the latter, it has been shown that neglecting interfacial effects can lead to erroneous predictions of the particle orientations while with the inclusion of capillarity, self-assemblies have been predicted in agreement with experimental observations [33,34,68].

In this Chapter we employ the simulated annealing method introduced in Ref. [68] to numerically study the equilibrium configuration of a single Janus particle adsorbed at a fluid-fluid interface. Our method takes into account the interfacial deformations so that we can predict the adsorption equilibrium configuration and the resulting induced interfacial deformation field. This last information is then exploited for predicting the capillary interactions between a pair of Janus particles at an interface. We compare our results with experimental findings concerning the self-assembly of charged Janus dumbbells at a water-decane interface, and find qualitative 


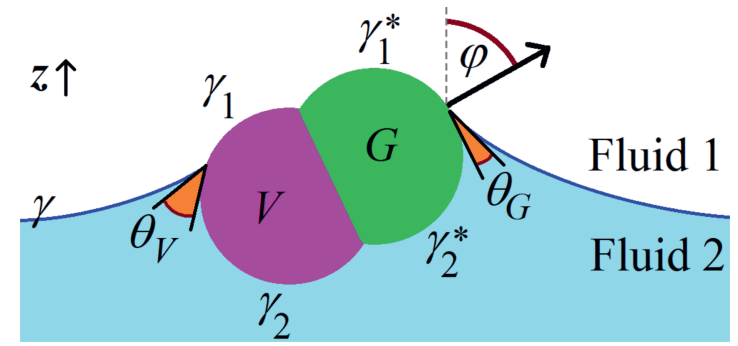

Figure 2.1: Sketch of a Janus particle at a fluid-fluid interface. The particle has two patches, indicated in green and violet. The various surface tensions are (see text) $\gamma, \gamma_{1}, \gamma_{2}, \gamma_{1}^{*}, \gamma_{2}^{*}$. The contact angles (measured inside fluid 2) of the fluid-fluid interface with the two patches, respectively $\theta_{G}$ and $\theta_{V}$ are given by Young's law (see Eq. (2.1)). In our model, the fluid-fluid interface coincides with a plane parallel to $z=0$ when no particle is adsorbed. The angle $\varphi$ is the polar angle of the particle vertical axis with respect to the fluid-fluid interface plane with $\varphi=\pi / 2$ corresponding to the particle horizontally aligned to the interface, $\varphi=0$ to the particle vertically aligned and with the green patch upwards, $\varphi=\pi$ to the particle vertically aligned and with the green patch downwards.

agreement.

This Chapter is organized as follows. In Section 2.2 we introduce the numerical method employed for our calculations. In Section 2.3 we study the adsorption of a single Janus particle at a fluid-fluid interface, i.e. we determine the equilibrium configuration and the resulting interfacial deformations. We then study the capillary pair interaction between two particles at an interface. In Section 2.4, we quickly review the experimental results and compare them with the numerical predictions. Finally, we present our conclusions in Section 2.5.

\subsection{Numerical method}

In this Section, we briefly illustrate the numerical method for our calculations. We consider two immiscible, homogeneous, and incompressible fluids separated by a fluid-fluid interface. We assume the fluid-fluid interface as a $2 \mathrm{D}$, possibly curved, surface, with zero thickness. When no particles are adsorbed, the fluid-fluid interface coincides with a plane parallel to $z=0$, where a Cartesian coordinate system $x, y, z$ has been introduced. At equilibrium, the shape of the fluid-fluid interface is given by the Young-Laplace Equation, with Young's law as a boundary condition for the contact angle [68]. The contact angle is the angle formed by the fluid-fluid interface along the three-phase contact line, i.e. where the fluid-fluid interface encounters any solid surface. When a Janus particle is adsorbed at the fluid-fluid interface, two different contact angles are formed by the fluid-fluid interface with the surface of the two patches of the particle, denoted here by green and violet patches, respectively, in Fig. 2.1. We call $\gamma$ the fluid-fluid surface tension between the two fluids, $\gamma_{1}, \gamma_{2}$ the surface tensions of the violet patch with fluid 1 and fluid 2, respectively, and $\gamma_{1}^{*}, \gamma_{2}^{*}$ the surface tensions of the green patch with fluid 1 and fluid 2, respectively. By convention, fluid 1 is the fluid at $z \rightarrow+\infty$ and fluid 2 the fluid at $z \rightarrow-\infty$. From Young's law [68], the contact angles $\theta_{V}$ and $\theta_{G}$, relative to the violet $(V)$ and green $(G)$ patch of the particle as indicated in Fig. 2.1, are given by

$$
\cos \theta_{V}=\frac{\gamma_{1}-\gamma_{2}}{\gamma} \quad, \quad \cos \theta_{G}=\frac{\gamma_{1}^{*}-\gamma_{2}^{*}}{\gamma}
$$


respectively. To study the adsorption of a Janus particle at a fluid-fluid interface, including the effects of capillary deformations, the equilibrium shape of the fluid-fluid interface, with respect to the particle position at the interface, is required. We compute the equilibrium shape using the numerical method introduced in Ref. [68]. In this method, the fluid-fluid interface is treated as a grid of points, and a simulated annealing algorithm [70] is used to find the point positions that minimize the thermodynamic potential ("energy") of the solid-fluid-fluid system, given the fixed position of the solid surfaces in the system as an input parameter. In our case, the solid surface position is defined by the positions and orientations of the $N$ particles adsorbed at the fluid-fluid interface. We define them by the $5 N$-dimensional vector $\Omega=\left(\Omega_{1}, \ldots \Omega_{N}\right)$, with $\Omega_{i}=\left(x_{i}, y_{i}, z_{i}, \varphi_{i}, \alpha_{i}\right)$ defining the configuration of the $i$-th particle $(i=1, \ldots N)$, where $x_{i}, y_{i}$ are the in-plane Cartesian coordinates of the center of mass, $z_{i}$ is the difference between the Cartesian coordinate $z$ of the center of mass and the interface height far away from the particle, $\varphi_{i}$ is the polar angle of the vertical axis with respect to the far-field interface normal (see Fig. 2.1 ), and $\alpha_{i}$ is the azimuthal angle of the particle vertical axis in the interface plane. Note that, since we consider particle shapes that are rotational invariant around their long axis, we do not need to specify a third Euler angle. The energy of the particles-fluid-fluid system can be written as $[68]$

$$
E_{N}(\Omega)=\gamma\left(S(\Omega)-A+W_{G}(\Omega) \cos \theta_{G}+W_{V}(\Omega) \cos \theta_{V}\right),
$$

where $S(\Omega)$ is the total fluid-fluid surface area, $W_{G}(\Omega)$ and $W_{V}(\Omega)$ are the total surface areas between fluid 1 and the green and violet patches, respectively. The constant $A$ is the planar fluid-fluid surface area when no particle is adsorbed, and is included in Eq. (2.2) to set the energy level $E_{N}=0$ when all particles are desorbed from the interface and are immersed in fluid 2.

For a given set of input parameters $\theta_{G}, \theta_{V}$ and $\Omega$, we compute first the equilibrium shape of the fluid-fluid interface using our numerical method. Once the equilibrium shape is known, we extract the minimum energy $E_{N}$ for these input parameters. By repeating this procedure for different $\Omega$ enables us to find the equilibrium particle configuration that minimizes the total energy of the system as well as the equilibrium shape of the fluid-fluid interface, including the equilibrium position of the three-phase contact line. The resulting contact angles, after the energy is minimized matches the input values $\theta_{G}, \theta_{V}$ used in Eq. (2.2). In order to mimic an infinitely flat interface far away from the particle, we place a solid vertical wall with contact angle $\pi / 2$ far away around our particle-fluid-fluid system to avoid particle-wall capillary interaction effects. In all the calculations presented in this work, the fluid-fluid interface shape that minimizes $E_{N}(\Omega)$ (Eq. (2.2)) is computed allowing fluid 1 and fluid 2 to exchange volume. So the minimum-energy level of the interface far away from the particles, for a given $\Omega$, is automatically found when the equilibrium shape of the interface is computed. More details on the numerical method can be found in Ref. [69]. Other examples of its application to colloidal particles at fluid-fluid interfaces and for a droplet in contact with heterogeneous surfaces can be found in Refs. [33, 34, 115-117].

\subsection{Numerical Results}

In this Section, we report our numerical results for the adsorption and capillary interactions of Janus colloidal particles at a fluid-fluid interface. First, the equilibrium orientation of a single adsorbed particle is shown, for different shapes and contact angles of the Janus particle. Then, 


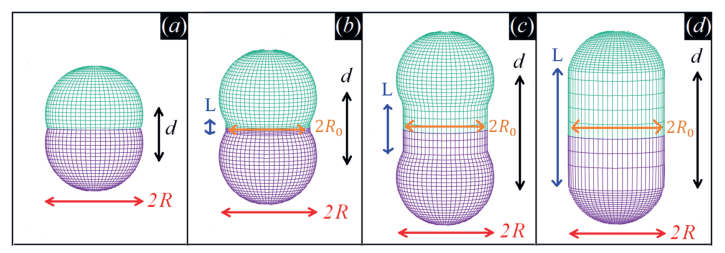

Figure 2.2: $(a)$ Janus dumbbell consisting of two interpenetrating spheres with radius $R$ and centerto-center distance $d=\sqrt{2} R$. (b) Janus dumbbell consisting of two interpenetrating spheres with radius $R$ and a small cylindrical part connecting them, with radius $R_{0}=R / \sqrt{2}$ and length $L=(1.7-\sqrt{2}) R$, for $d=1.7 R$. (c) Janus dumbbell consisting of two touching spheres with radius $R$ and a cylindrical part connecting them, with $R_{0}=R / \sqrt{2}$ and $L=(2-\sqrt{2}) R$, for $d=2 R$. (d) The limiting case of a Janus spherocylinder of two touching spheres of radius $R$ and a cylindrical part of length $L$ with $2 R_{0}=L=d=2 R$.

the interfacial deformations induced by the adsorbed particle are analyzed. Finally, we study the capillary pair interaction between two identical particles adsorbed at the interface. The shapes of the Janus particles considered in this work are shown in Fig. 2.2: $(a)$ a dumbbell of two spheres of radius $R$ and center-to-center distance $d,((b)-(c))$ a dumbbell with $(b)$ a short and $(c)$ a long inter-sphere cylindrical neck of radius $R_{0}<R$ and length $L$, and $(d)$ a spherocylinder of cylinder length $L$ and radius $R_{0}=R$. The Janus character of all four particles $(a)-(d)$ is also illustrated in Fig. 2.2, i.e. such that the uniaxial character of the particles is preserved.

\subsubsection{Single adsorbed particle}

Here we present the equilibrium orientation of a single-adsorbed Janus particle, for each of the particle shapes shown in Fig. 2.2, and for various values of the contact angles $\theta_{G}$ and $\theta_{V}$ of the two particle patches. Next, we analyze the interfacial deformation field induced by each particle when adsorbed in equilibrium.

The configuration of a single-adsorbed particle at the interface, using the notation of Section 2.2, is $\Omega=(x, y, z, \varphi, \alpha)$, where the subscript " 1 " is omitted since we consider a single particle (i.e. $N=1)$. In Fig. 2.3( $(a)-(d))$, we show a $3 \mathrm{D}$ view of the equilibrium adsorption configuration of a Janus particle, for the different shapes shown in Fig. 2.2 and for $\cos \theta_{G}=-\cos \theta_{V}=-0.4$. The equilibrium values $z^{*}$ and $\varphi^{*}$ of the particle height $z$ measured with respect to the interface level far away from the particle and of the particle orientation $\varphi$ are reported for each shape. Since we consider a single-particle at a planar fluid-fluid interface, $x, y$, and $\alpha$ of the particle are irrelevant by symmetry. The dumbbell (shape ( $a$ ) of Fig. 2.2) prefers the vertically aligned orientation, with the green patch completely immersed in fluid 1 and the violet patch completely immersed in fluid 2. The other three particle shapes, $(b),(c)$ and $(d)$ of Fig. 2.2, instead, prefer a tilted orientation, with the green patch predominantly immersed in fluid 1 and the violet patch largely immersed in fluid 2. The equilibrium polar tilt angle $\varphi^{*}$ increases monotonically from case $(a)$ to case $(d)$. This is due to the fact that for a longer cylindrical part of the particle, the surface area that is excluded from the fluid-fluid interface increases, when the particle is more tilted. As a consequence, the tilt of the adsorbed particle increases with the length of the cylindrical neck of the particle. For each of the four cases shown in Fig. 2.3 $((a)-(d))$, we report in Fig. 2.3((e)-(h)) a contour plot of the fluid-fluid interface height profile, as computed 

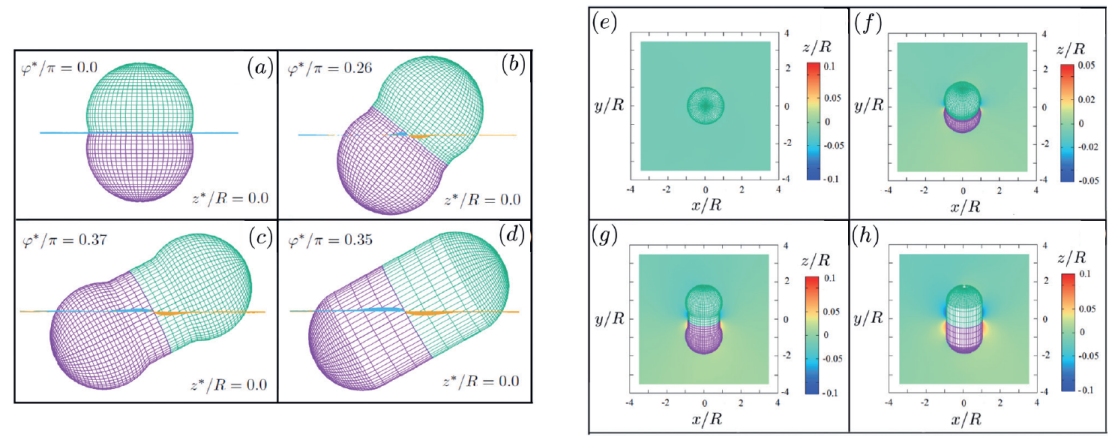

Figure 2.3: $3 \mathrm{D}$ views $((a)-(d))$ and contour plots $((e)-(h))$ of the equilibrium configuration of a Janus particle with the shapes shown in Fig. 2.2 at a fluid-fluid interface, and with contact angles of the green and violet patches (see Fig. 2.1) given by $\cos \theta_{G}=-\cos \theta_{V}=-0.4$. While the equilibrium particle height on the interface level far away from the particle is, as expected, $z^{*}=0$, in all cases, the orientation $\varphi^{*} / \pi$ depends on the particle shape. In cases $(a)$ and $(e) \varphi^{*} / \pi=0$, and no interfacial deformations are induced. In all the other cases, the particle is tilted with respect to the interface and the interfacial deformation field is dominated by a hexapolar mode with increasing intensity upon increasing the length of the cylindrical part of the Janus dumbbell. The hexapolar mode is maximum in the case of the spherocylinder. This case is analyzed in detail in Section 2.3.2.

through our method. The dumbbell in its equilibrium adsorption configuration does not deform the interface, while an asymmetric hexapolar capillary deformation is found in the other three cases, as will be discussed in more detail in the next Section.

As the interfacial deformation field is most pronounced for the Janus spherocylinder, we will use this particle shape to investigate the role of the contact angles $\theta_{G}$ and $\theta_{V}$. In Fig. 2.4 we show a $3 \mathrm{D}$ view of the equilibrium configuration of a spherocylinder (shape $(d)$ of Fig. 2.2) for $\cos \theta_{G}=-0.4,0,0.4$ and $\cos \theta_{V}=-0.4,0,0.4$. In the graph of Fig. 2.4, the diagonal cases with $\cos \theta_{G}=\cos \theta_{V}$ correspond to the cases of a homogeneous (i.e. non-Janus) spherocylinder. The other cases of Fig. 2.4 are symmetric with respect to this diagonal, with the green and violet patch exchanging role (since their contact angles are interchanged). In the case $\cos \theta_{G}=$ $\cos \theta_{V}=0$, the equilibrium height of the spherocylinder's center of mass at the interface level is $z^{*}=0$, the spherocylinder prefers the horizontal orientation, i.e. $\varphi^{*} / \pi=0.5$, and no interfacial deformations are observed, i.e. the interface remains flat also in the presence of the particle. When $\cos \theta_{G}=\cos \theta_{V}= \pm 0.4$, the spherocylinder is also horizontal at the interface, but $z^{*} / R=\mp 0.40$. Note that the equilibrium heights are of the same magnitude, since these cases are symmetric with respect to $z$ (inverting the sign of the cosine of the contact angle is equivalent to exchanging fluid 1 and fluid 2, see Eq. (2.1)). Along the diagonal, where $\theta_{G}=\theta_{V}$, the interfacial height profile is flat as expected [118]. In all the off-diagonal cases, the spherocylinder at the equilibrium stays tilted at the interface, i.e. $\varphi^{*} / \pi \neq 0.5$. In particular, the bigger $\left|\cos \theta_{G}-\cos \theta_{V}\right|$, the more the spherocylinder is tilted. Most interestingly, in all the off-diagonal cases, i.e. where the spherocylinder is a Janus particle, the interfacial deformation field is dominated by a hexapolar mode, rather than by a flat interface as observed for the non-Janus spherocylinders. We will discuss this in detail in the next Section.

Analogous graphs to Fig. 2.4 are reported in Fig. 2.5, for the other three particle shapes which show that the intensity of the interfacial deformation field decreases upon decreasing the length 


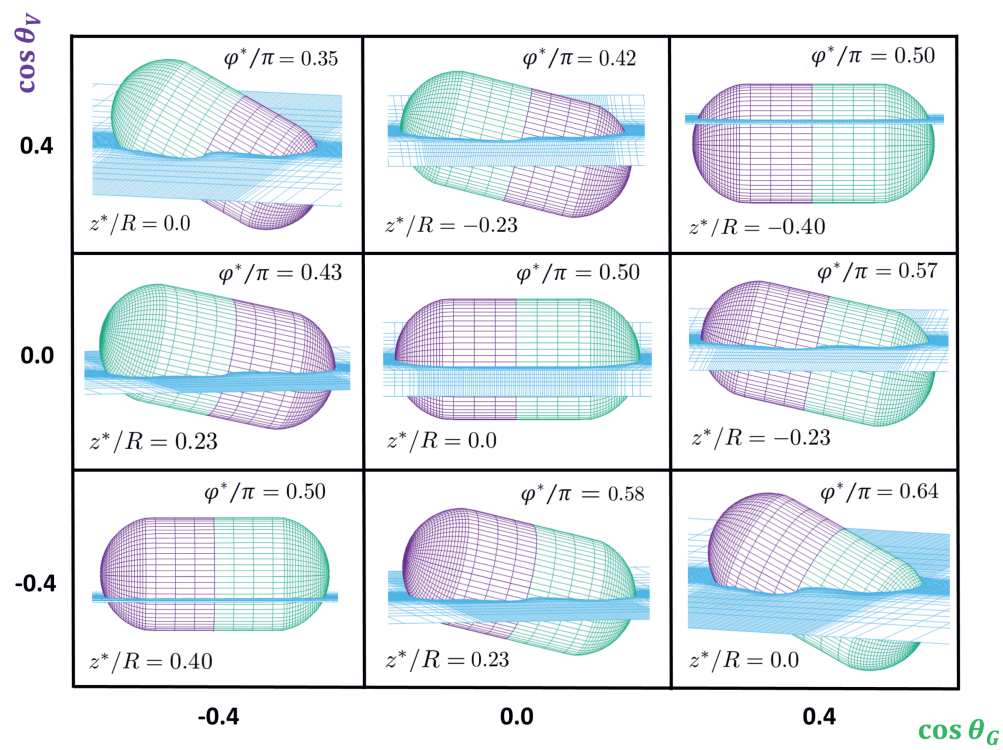

Figure 2.4: 3D views of the equilibrium configurations of a Janus spherocylinder (shape $(d)$ in Fig. 2.2 ) adsorbed at a fluid-fluid interface, with varying contact angles $\theta_{G}$ and $\theta_{V}$. The graph is symmetric with respect to the diagonal corresponding to the cases of a homogeneous (non-Janus) spherocylinder, i.e. $\theta_{G}=\theta_{V}$. When $\cos \theta_{G}=\cos \theta_{V}=0, \varphi^{*} / \pi=0.5, z^{*}=0$, no interfacial deformations are induced in the fluid-fluid interface shape. For $\cos \theta_{G}=\cos \theta_{V}= \pm 0.4, \varphi^{*} / \pi=0.5, z^{*} / R=\mp 0.40$, the induced interfacial deformation fields are flat as expected [118]. In all the off-diagonal cases, the deformation field is dominated by the hexapolar mode, whose intensity is proportional to $\left|\cos \theta_{G}-\cos \theta_{V}\right|$. This is shown in detail in Section 2.3.2. 

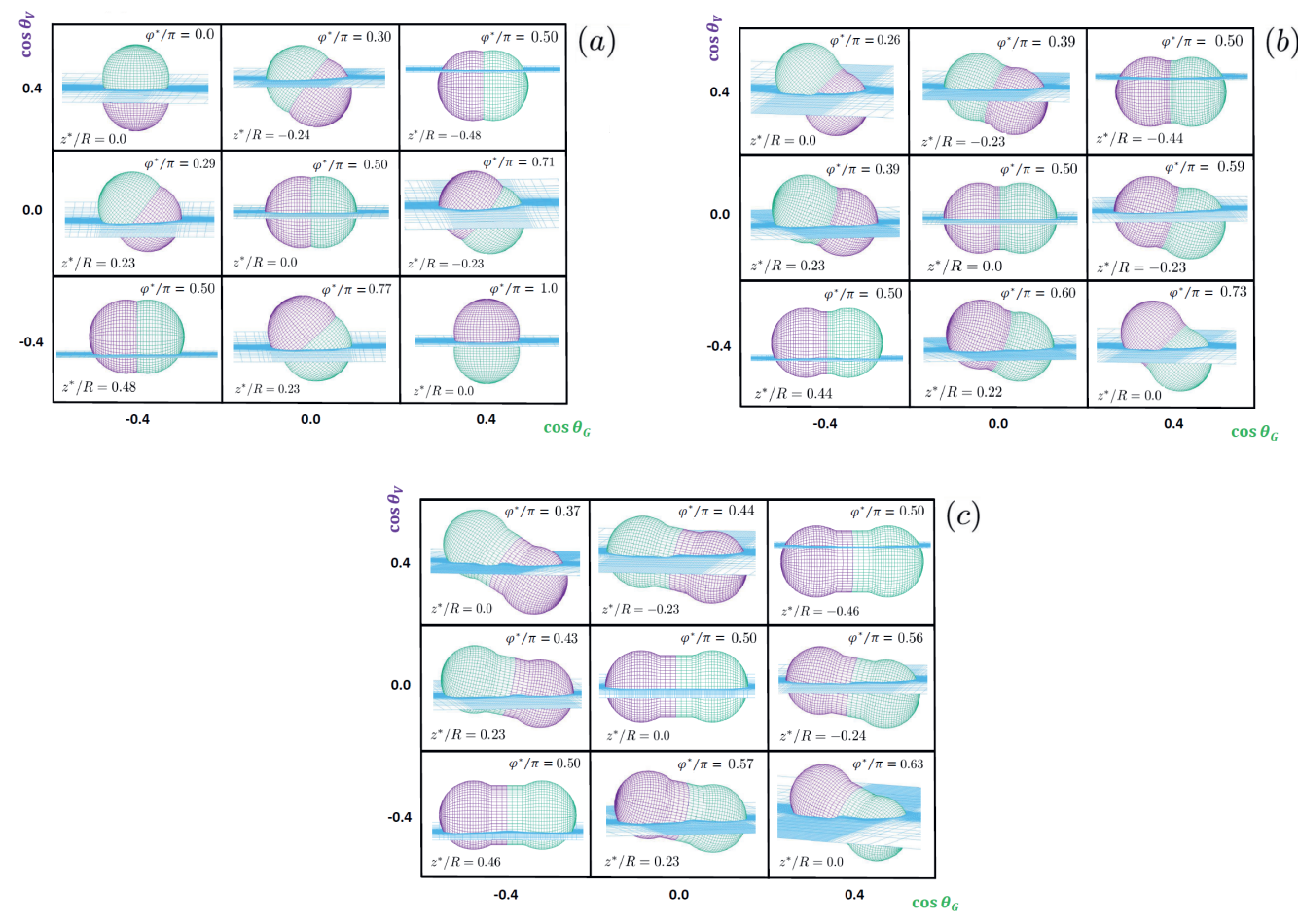

Figure 2.5: $3 \mathrm{D}$ views of the equilibrium configurations of a series of Janus dumbbells (see Fig. 2.2) adsorbed at a fluid-fluid interface, with varying contact angles $\theta_{G}$ and $\theta_{V}$. Each graph is symmetric with respect to the diagonal corresponding to the cases of a homogeneous (non-Janus) dumbbell, i.e. $\theta_{G}=\theta_{V}$. (a) In all the cases we do not observe any interfacial deformation. (b) When $\cos \theta_{G}=$ $\cos \theta_{V}=0, \varphi^{*} / \pi=0.5, z^{*}=0$, no interfacial deformations are induced in the fluid-fluid interface shape. For $\cos \theta_{G}=\cos \theta_{V}= \pm 0.4, \varphi^{*} / \pi=0.5, z^{*} / R=\mp 0.44$, the induced interfacial deformation fields are flat as expected [118]. In all the off-diagonal cases, the deformation field is dominated by the hexapolar mode, whose intensity is proportional to $\left|\cos \theta_{G}-\cos \theta_{V}\right|$. (c) When $\cos \theta_{G}=\cos \theta_{V}=$ $0, \varphi^{*} / \pi=0.5, z^{*}=0$, no interfacial deformations are induced in the fluid-fluid interface shape. For $\cos \theta_{G}=\cos \theta_{V}= \pm 0.4, \varphi^{*} / \pi=0.5, z^{*} / R=\mp 0.46$, the induced interfacial deformation fields are flat as expected [118]. In all the off-diagonal cases, the deformation field is dominated by the hexapolar mode, whose intensity is proportional to $\left|\cos \theta_{G}-\cos \theta_{V}\right|$. 

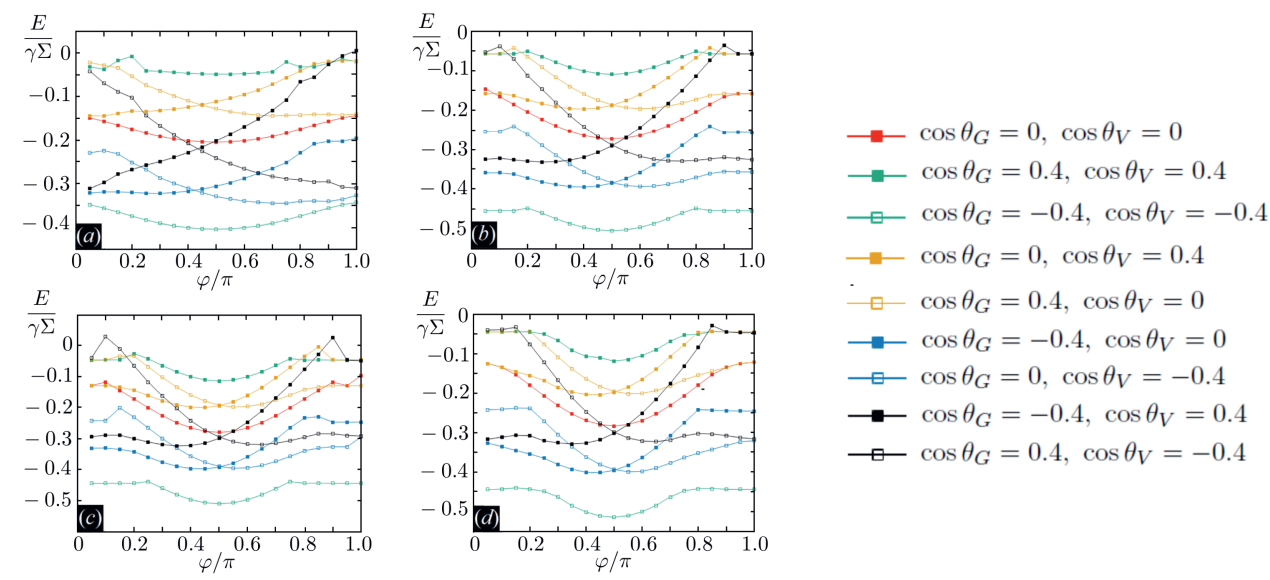

$\square \cos \theta_{G}=0.4, \cos \theta_{V}=0$

$\longrightarrow \cos \theta_{G}=-0.4, \cos \theta_{V}=0$

$\because \cos \theta_{G}=0, \cos \theta_{V}=-0.4$

$\because \cos \theta_{G}=0.4, \cos \theta_{V}=-0.4$

Figure 2.6: Adsorption energy E (Eq. (2.2) of Section 2.2) for all the particles shapes considered in this Chapter (see Fig. 2.2) as a function of the polar angle $\varphi$ at fixed combinations of the contact angles $\theta_{G}$ and $\theta_{V}$. Here $E$ is plotted in units of $\Sigma \gamma$ with $\gamma$ the fluid-fluid surface tension and $\Sigma$ the surface area of the particles. Observe that, as in Ref. [34], in our case $E$ is already minimized with respect to the particle height $z$.

of the cylindrical part of the Janus dumbbell. Also, in Fig. 2.6, we report the energy $E_{1}$ (Eq. (2.2)) computed by our numerical method for all particle orientations $\varphi$ considered, and for all the particle shapes and contact angle values considered. Since we perform calculations for many particle shapes and contact angles we consider a relatively large step size $\Delta \varphi=0.05 \pi$ and a relatively large grid spacing of $0.007 \mathrm{R}$.

\subsubsection{Fit of the interfacial deformation field}

Within the approximation of small interfacial deformations, the equilibrium height profile of a fluid-fluid interface around a colloidal particle centered in the origin can be written as $[85,92]$

$$
\frac{h(r, \phi)}{R}=C_{0} \ln \left(\frac{r}{R}\right)+\sum_{m=1}^{\infty} C_{m} \frac{\cos \left(m\left(\phi-\phi_{m}\right)\right)}{(r / R)^{m}}
$$

where we introduced the cylindrical coordinates $r$ and $\phi$, with $r=\sqrt{x^{2}+y^{2}}$ and $\phi=\arctan \left(\frac{y}{x}\right)$, and the fluid-fluid interface far away from the particle is at $z=0$. In Eq. (2.3), the coefficients $C_{0}, C_{m}$ and $\phi_{m}$ are referred to, respectively, as the monopole $\left(C_{0}\right)$, the dipole $\left(C_{1}\right)$, the quadrupole $\left(C_{2}\right)$, the hexapole $\left(C_{3}\right)$, etc. We consider the case of the Janus spherocylinder with $\cos \theta_{G}=-\cos \theta_{V}=0.4$, as shown in Figs. 2.3(d) and 2.3(h). In Fig. 2.7, we report the height profile $h(r, \phi) / R$ as a function of $\phi$ for the two distinct values $r / R=2$ and $r / R=5$ as computed through our numerical method. The fit shown in the figure is obtained by the multipole expansion Eq. (2.3) with the coefficients as reported in Table 2.1, and neglecting the modes with $m \geq 4$.

The monopolar and dipolar modes are not expected for a particle at its equilibrium adsorption configuration, unless external forces are applied to the particle [92]. In our case these modes approach zero when a very accurate equilibration of the particle configuration (step 

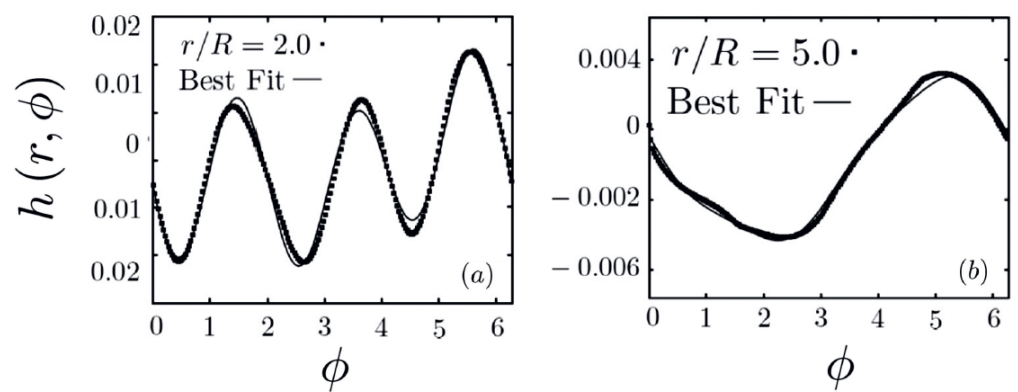

Figure 2.7: Interface height profile $h(r, \phi)$ around the Janus sperocylinder at its equilibrium adsorption configuration in the case shown in Figs. 2.3 $(d)$ and 2.3 $h$ ), as a function $\phi$, and for the two distinct values of $r / R=2$ and $r / R=5$, where $r$ and $\phi$ are cylindrical coordinates (see text). The fit shown in the figure is the multipole expansion Eq. (2.3) with the coefficients as reported in Table 2.1. The dots represent our numerical solution.

size $\Delta \varphi=0.001 \pi$ and grid spacing $0.0001 R$ ) is performed. However, due to the precision of our numerical method these modes never disappear completely. Nevertheless their amplitudes $C_{0} \approx-0.00071$ and $C_{1} \approx-0.0063$ are much smaller than the one of the hexapolar mode $C_{3} \approx-0.069$. Additionally the quadrupolar mode $C_{2} \approx-0.0072$ is also much weaker than $C_{3}$ and thus the hexapolar mode is the dominant mode. In Fig. 2.7 the combination of the hexapolar and quadrupolar modes can only be appreciated at small distances $(r / R=2)$, since at large distances $(r / R=5)$ only the lower order terms of Eq. (2.3) survive. The deviation of the fit from the numerically obtained one for small $r / R$ is caused by neglecting higher-order modes with $m \geq 4$ in Eq. (2.3).

Table 2.1: Coefficients $C_{m}$ of the multipole expansion (2.3) obtained through a fit of the numerical obtained results. Here $C_{0}, C_{1}, C_{2}$ and $C_{3}$ are the amplitudes of the monopolar, dipolar, quadrupolar and hexapolar modes, respectively, while $\phi_{1}, \phi_{2}$ and $\phi_{3}$ are their phases

\begin{tabular}{lll}
\hline$m$ & $100 \cdot C_{m}$ & $\phi_{m}$ \\
\hline 0 & $-0.071 \pm 0.002$ & - \\
1 & $-0.63 \pm 0.03$ & $2.26 \pm 0.01$ \\
2 & $-0.72 \pm 0.2$ & $0.87 \pm 0.23$ \\
3 & $-6.9 \pm 1.0$ & $1.26 \pm 0.01$ \\
\hline
\end{tabular}

\subsubsection{Capillary pair interaction}

After showing the equilibrium configuration of a single-adsorbed Janus particle and the induced deformation field, we consider the capillary pair interactions between two identical Janus particles adsorbed at the interface. Following Ref. [33], we define the capillary interaction energy per particle as

$$
\tilde{E}_{N} \equiv \frac{E_{N}}{N}-E_{1}
$$

where $N$ is the number of adsorbed particles and $E_{N}$ is the energy of the fluid-fluid-particles system numerically computed through the method discussed in Section 2.2. Note that $\tilde{E}_{N}=0$ 
when only one particle is adsorbed $(N=1)$, or when $N$ particles are adsorbed, but so far apart that they are not interacting, i.e. $E_{N}=N E_{1}$. Here we are interested in the pair interaction, $N=2$. In Fig. 2.8, we report the interaction energy per particle $\tilde{E}_{2}$ for two adsorbed Janus spherocylinders with contact angles $\cos \theta_{G}=-\cos \theta_{V}=-0.4$, as a function of the distance

$D \equiv \sqrt{\left(x_{1}-x_{2}\right)^{2}+\left(y_{1}-y_{2}\right)^{2}}$ between their centers of mass. The height of the particles at the interface level is $z_{1}=z_{2}=z^{*}$, and their orientation is $\varphi_{1}=\varphi_{2}=\varphi^{*}$, with $z^{*}$ and $\varphi^{*}$ the equilibrium values found for this particle shape and contact angles (see Section 2.3). The relative azimuthal angle of the two particles in the interface plane is set to $\alpha_{1}-\alpha_{2}=0, \pi$, corresponding to a laterally aligned and an anti-aligned configuration, respectively, see Fig. 2.8. We find attractive capillary interactions for the laterally aligned configuration (black), and repulsive capillary interactions for the anti-aligned configuration (orange). Interestingly, this result is only expected when the dominant mode of the interfacial deformation field induced by the two particles is a hexapole, as in our results. Instead, if the dominant mode had been a quadrupole, attractive capillary interactions would have been found for both laterally aligned and antialigned configurations. This is a key finding of this Chapter that allows us to experimentally distinguish particles with quadrupolar and hexapolar deformation fields, as we will discuss in Section 2.4. We also mention here that the residual dipolar mode found in the previous paragraph does not significantly affect the pair interaction between the spherocylinders. As shown in Fig. 2.7, the dipole still survives at a distance $r / R=5$ from a single spherocylinder while, as shown in Fig. 2.8, the interaction energy per particle is negligible, if compared to the near contact value, at a distance $D / R \approx 3.6$ between two spherocylinders.

A graph analogous to the one in Fig. 2.8 is presented in Fig. 2.9, for the tip-tip interactions. In this case, within the precision of our numerical method, we do not observe any interaction between the spherocylinders.

Note that the pair potential $\tilde{E}_{2}$ in Fig. 2.8 is plotted in units of $\gamma \Sigma$, where $\Sigma$ is the total surface area of a spherocylinder. For typical values $\gamma \sim 10^{-2} \mathrm{Nm}^{-1}$ and micrometer-sized areas $\Sigma \sim 10^{-12} \mathrm{~m}^{2}$, we have $\gamma \Sigma \sim 10^{-14} \mathrm{~J} \sim 10^{7} k_{B} \mathrm{~T}$, such that the capillary pair interaction at contact, $\tilde{E}_{2} \sim\left(10^{-4}-10^{-3}\right) \gamma \Sigma$ in Fig. 2.8 corresponds to strong capillary interactions of hundreds of $k_{B} T$.

We consider the case of a Janus spherocylinder here as the numerical result is the most accurate for this particle shape due to the high intensity of the interfacial deformation field. We expect similar, but weaker, capillary interactions for the other particle shapes of Figs. $2.2((b)-(c))$ as the deformation fields are qualitatively similar but less pronounced.

\subsection{Comparison wih experiments}

In this Section we review a series of experimental findings concerning the adsorption and selfassembly of charged Janus dumbbells at a water-decane interface. These experiments have been performed by Dr. F. Chang in the group of Prof. W. K. Kegel and full details can be found in Refs. $[29,30]$. We then present a comparison between experimental findings and the theoretical predictions of the previous Sections.

\subsubsection{Chains of dumbbells}

Micrometer-sized charged Janus dumbbells were synthesized following the procedure described in Refs. $[29,30]$. After that, the dumbbells were spread onto the water-decane interface, and 


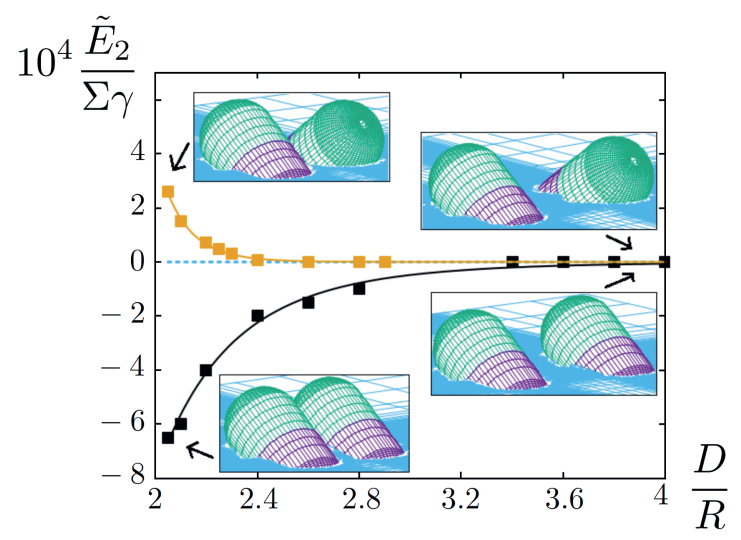

Figure 2.8: Interaction energy per particle $\tilde{E}_{2} / \Sigma \gamma$ (Eq. (2.4) in Section 2.3.3) of two parallel Janus spherocylinders, adsorbed in their equilibrium configuration, as a function of the distance $D$ between their centers of mass, for laterally aligned (black) and anti-aligned (orange) orientations. The total surface area is denoted as $\Sigma$ and the fluid-fluid surface tension is $\gamma$. The contact angles of the Janus spherocylinders are given by $\cos \theta_{G}=-0.4$ (in green) and $\cos \theta_{V}=0.4$ (in violet).
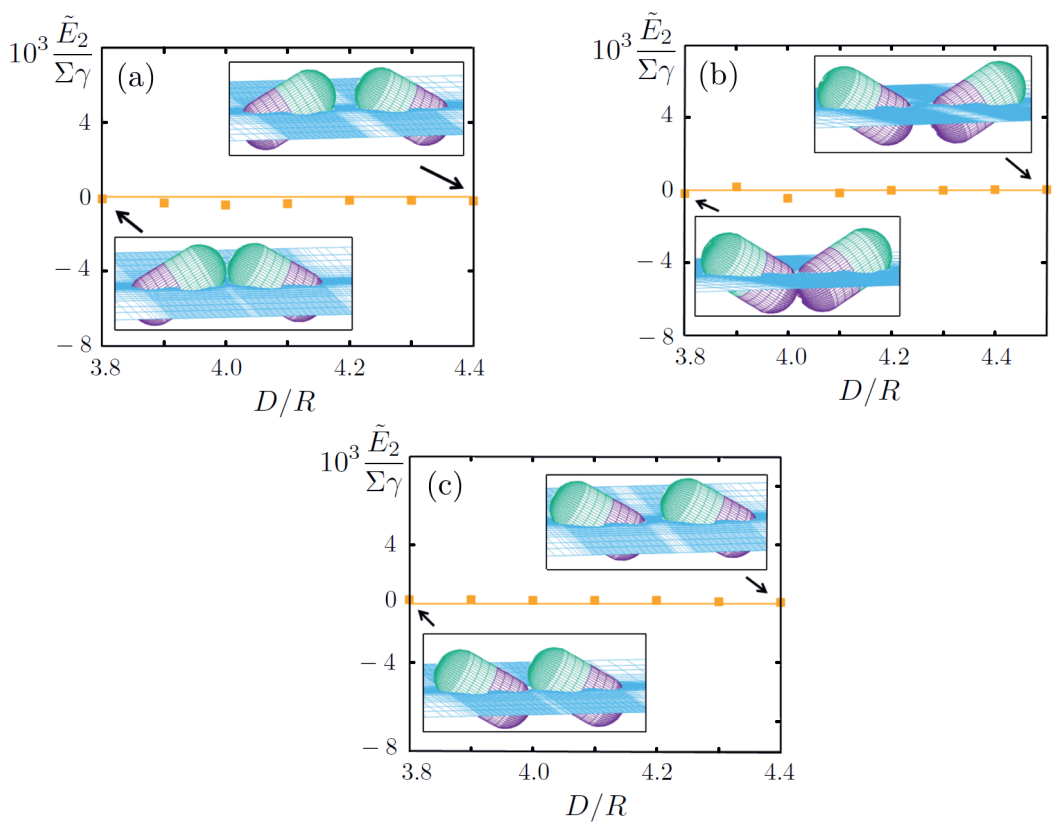

Figure 2.9: Same as in Fig. 2.8, but with the spherocylinders approaching their "heads" rather than their lateral sides. We do not obserseve any interaction between the spherocylinders. 


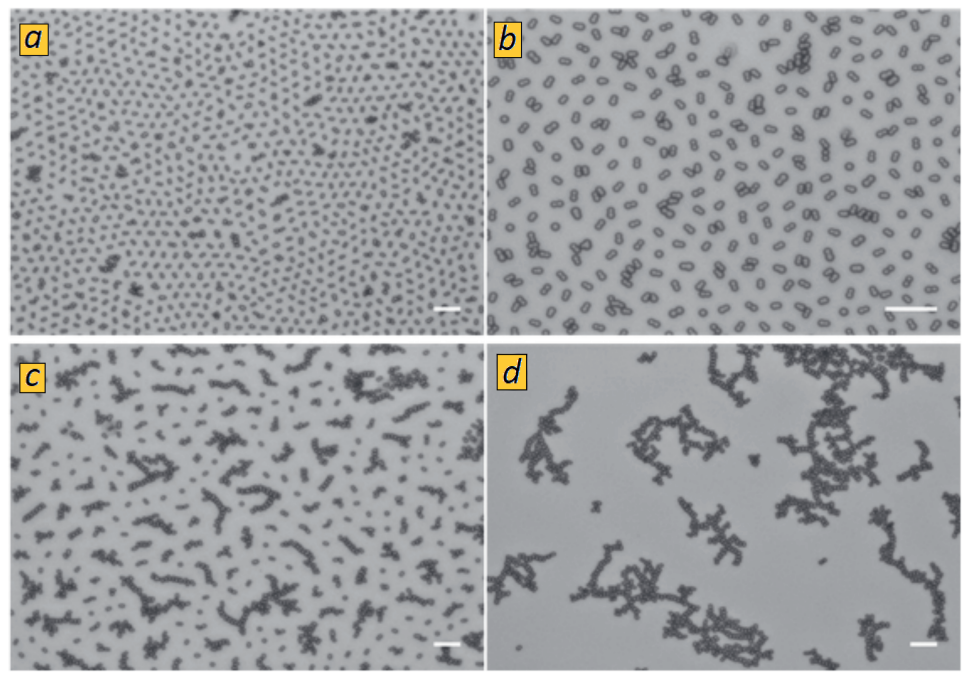

Figure 2.10: The structure evolution in a monolayer containing charged Janus dumbbells at the water-decane interface: (a) microstructure obtained right after spreading particles at the interface; after (b) 25 hours; $(c) 50$ hours; $(d) 120$ hours. The scale bar corresponds to $20 \mu \mathrm{m}$. Experiments were performed by Dr. F. Chang in the group of Prof. W. K. Kegel.
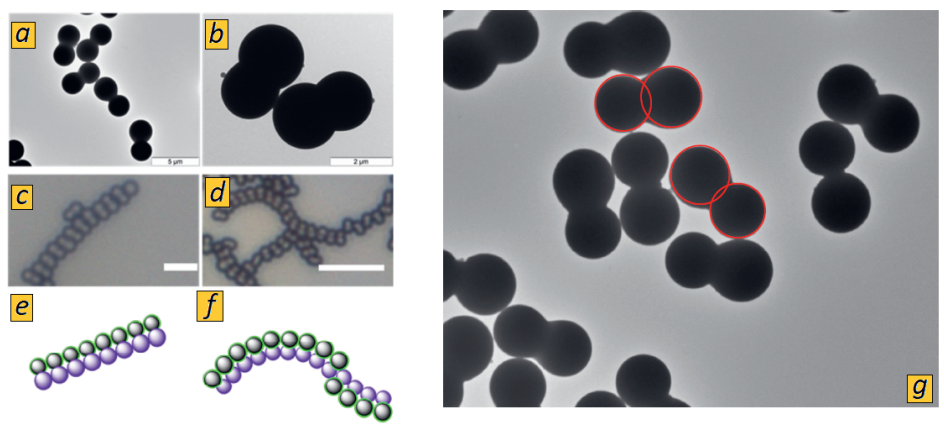

Figure 2.11: Transmission electron microscope images of symmetric Janus dumbbells $(a)$ and slightly asymmetric Janus dumbbells (b); a linear string of symmetric Janus dumbbells $(c)$ and a curved chain of slightly asymmetric Janus dumbbells $(d)$ formed at an interface. In case $(c)$ and $(d)$ the scale bar corresponds to $10 \mu \mathrm{m}$. Schematic images of the observed structures: a linear string of laterally aligned symmetric Janus dumbbells (e), a curved chain of laterally aligned and slightly asymmetric Janus dumbbells $(f)$. As shown in $(g)$, the shape of the symmetric Janus dumbbells can best be compared to the dumbbells of Figs. 2.2(b) and 2.2(c), i.e. dumbbells consist of smoothly joined, interpenetrating or touching spheres. Experiments were performed by Dr. F. Chang in the group of Prof. W. K. Kegel. 
the structural time evolution of the monolayer was monitored with optical microscopy. Fig. $2.10(a)$ depicts the initial configuration of the microstructure. The charged dumbbells at the interface give rise to an ordered crystalline monolayer with a few aggregates present, similar to the well-known behavior observed by Pieranski for charged spheres [12]. This is attributed to the dipole-dipole repulsions from the charges on both lobes of the dumbbells. The particles assemble predominantly by connecting their waists, thereby forming lateral structures (see Fig. $2.10(b)$ ). Only a few tip-tip configurations were observed which are mainly due to the roughness of the seed lobes during the seeded dispersion polymerization process. The linear chains grow in time until they encounter each other to form interconnected aggregates and branched structures as shown in Fig. 2.10(c). Because of the presence of electrostatic interactions, the structural evolution proceeds relatively slowly. Fig. 2.10 $(d)$ shows a network structure consisting of rigid linear segments, and this system evolved to such a state in about 120 hours after spreading. A zoomed image of the observed lateral structures is shown in Fig. 2.11(c).

Fig. 2.10 also shows that the particles first form doublets and only at a later stage longer chains, which is expected for charged particles.

\subsubsection{Discussion and comparison with theoretical predictions}

Even if electrostatic forces are present in our system, capillarity is still expected to play a major role in the self-assembly process as was also observed in Ref. [23]. Therefore a qualitative comparison between our experimental analysis and the numerical predictions should be justified.

As shown in Fig. 2.11 $(\mathrm{g})$, the shape of our experimentally synthesized dumbbells can best be compared to the dumbbells of Figs. 2.2(b) and 2.2(c), rather than to the ones of Fig. 2.2(a), i.e. dumbbells consist of smoothly joined, interpenetrating or touching spheres.

The structures experimentally observed can be explained by an induced deformation field dominated by a hexapolar mode, as predicted by our numerical calculations. Indeed the lateral aggregates experimentally observed, consist of only laterally aligned configurations of two neighboring particles and never of laterally anti-aligned configurations. Actually this conclusion cannot be inferred from the analysis performed on the symmetric Janus dumbbells. In this case it is impossible to distinguish which lobe of the dumbbell is hydrophilic and which one is hydrophobic so that even if lateral structures are observed, it is not possible to say if they consist of laterally aligned configurations or of laterally anti-aligned ones. It then follows that it is not possible to distinguish between a hexapolar induced capillary deformation field and a quadrupolar one. To clarify this point, slightly asymmetric dumbbells (see Fig. 2.11(b)) were used as reference. In the case of asymmetric dumbbells, it is possible to distinguish the hydrophobic lobe of the dumbbell from the hydrophilic one since one lobe is slightly bigger than the other. As shown in Fig. 2.11(d), asymmetric dumbbells evolve into curved chains in which the "big" lobe of each particle attracts the "big" lobe of the neighboring particle. The same of course happens between the "small" lobes. This indicates that the chains consist of laterally aligned configurations and since the dumbbells are just slightly asymmetric, we expect this to also hold for less asymmetric and even symmetric dumbbells. A sketch of the structures observed in these two cases is depicted in Figs. 2.11(e) and 2.11(f). As explained in Section 2.3.3, this finding allows us to conclude that the capillary deformation field is hexapolar, in agreement with our numerical predictions. 


\subsection{Conclusions}

In this Chapter, we numerically studied the adsorption and the self-assembly at a fluid-fluid interface of a variety of anisotropic Janus particles interpolating between a pair of interpenetrating equal-sized spheres to a spherocylinder by the insertion of a cylindrical neck. Each particle shape has two distinct "faces" with contact angles $\theta_{G}$ and $\theta_{V}$, respectively. Our study was performed using a numerical method that takes into account the interfacial deformations. For (Janus or non-Janus) dumbbells consisting of interpenetrating spheres, we always find a flat interface. For dumbbells with a cylindrical neck and for spherocylinders, we find a flat interface in the non-Janus case $\left(\cos \theta_{G}=\cos \theta_{V}\right)$, and that the deformation field is dominated by an hexapolar mode in the Janus case $\left(\cos \theta_{G} \neq \cos \theta_{V}\right)$. The intensity of this hexapole is proportional to the dimension of the cylindrical neck between the spheres composing the particles and to the difference between their contact angles. The intensity of the hexapole is hence strongest in the case of Janus spherocylinders when $\left|\cos \theta_{G}-\cos \theta_{V}\right|$ is maximum. We then found that the hexapolar field induces capillary attractions between two laterally aligned Janus spherocylinders at the interface and repulsions for two laterally anti-aligned ones. For micrometer-sized spherocylinders this attraction is of the order of hundreds $k_{B} T$ and since the other particle shapes are expected to induce qualitatively the same deformation field, we expect qualitatively the same capillary interactions, although a bit weaker. Our numerical predictions qualitatively explain the formation of structures of laterally aligned Janus dumbbells, recently experimentally observed at a water-decane interface.

\subsection{Acknowledgments}

The results presented in this Chapter are part of a collaboration with Dr. F. Chang, who performed the experimental work described here in the group of Prof. W. K. Kegel, and Dr. G. Soligno, who provided the numerical code in the initial stage of the project. 



\section{Chains of Cubic Colloids at Fluid-Fluid Interfaces}

Inspired by recent experimental observations of spontaneous chain formation of cubic particles adsorbed at a fluid-fluid interface, we theoretically investigate whether capillary interactions can be responsible for this self-assembly process. We calculate adsorption energies, equilibrium particle orientations, and interfacial deformations, not only for a variety of contact angles but also for single cubes as well as an infinite 2D lattice of cubes at the interface. This allows us to construct a ground-state phase diagram as a function of areal density for several contact angles, and upon combining the capillary energy of a 2D lattice with a simple expression for the entropy of a 2D fluid we also construct temperature-density or size-density phase diagrams that exhibit large two-phase regions and triple points. We identify several regimes with stable chainlike structures, in line with the experimental observations. 


\subsection{Introduction}

Capillary-driven self-assembly of colloidal particles trapped at a fluid-fluid interface has recently gained a great deal of attention in the soft-matter community [119-121]. Indeed, by controlling the strength and the directionality of capillary interactions, quasi-2D ordered structures at interfaces can be obtained, which have interesting applications ranging from the stabilization of foams and Pickering emulsions [16] to the development of new materials [17]. Several strategies to control capillarity have been elaborated in recent years. These include the use of particles with surface chemical heterogeneities, i.e. patchy and Janus particles [26-28, 100,122], the application of external electric or magnetic fields [123], and the employment of interfaces with a finite curvature [87]. Another popular method to control capillarity consists of considering particles with anisotropic shapes. Indeed, because of the constraint imposed by Young's law [13], nonspherical colloidal particles adsorbed at a fluid-fluid interface induce interfacial deformations that determine their orientations and generate mutual capillary interactions. Different anisotropic shapes induce different deformation fields such that the particles shape plays a key role in regulating the self-assembly of colloids trapped at an interface.

Since a large variety of colloidal particles is nowadays available, with tunable shapes and surface functionalizations $[8,18]$, the behavior at interfaces of $\mathrm{nm}$ - and $\mu \mathrm{m}$-sized particles with several anisotropic shapes, e.g. rods, cylinders, cubes, dumbbells and discs [19-25] has been widely studied in the past few years. Among the several shapes studied so far, cubic particles have been proven to form $2 \mathrm{D}$ structures with striking features. In recent experiments [31,32] for example, polystyrene cubes with $30 \mu \mathrm{m}$ edge length have been reported to self-assemble at a water-air interface into three distinct structures, namely square close-packed, linear, and hexagonal structures. Furthermore, the relative stability of these three phases has been shown to strongly depend on their wettability, which is specified by the so-called Young contact angle $\theta$ [13]. While hydrophilic cubes with a small $\theta \sim 0$ give rise to square close-packed aggregates, by increasing $\theta$ first linear aggregates and finally hexagonal aggregates are observed. Cases of coexistence between linear and hexagonal aggregates have also been reported [31,32].

By noting that the cubes display different single-particle configurations in the different observed structures, i.e. a "face up" configuration in the square close-packed structure, an "edge up" configuration in the linear structure, and a "vertex up" configuration in the hexagonal structure, the authors of Refs. [31,32] have argued that the formation of each 2D structure could be caused by a different kind of deformation field induced by the cubes at the interface. In particular these authors have proposed that cubes adsorbed in the "face up", "edge up", and "vertex up" configurations would induce monopolar, quadrupolar and hexapolar deformation fields, respectively.

The scenario experimentally found in Refs. [31,32] represents, at the same time, a confirmation and an extension of the theoretical predictions previously presented in Refs. [33,34]. Here it was shown that cubic colloidal particles with a Young contact angle $\theta=\pi / 2$ indeed adsorb at a fluid-fluid interface with one vertex pointing upward with respect to the interface plane ("vertex up" configuration), and induce a hexapolar deformation field in the interface height profile which causes nm-sized cubes to self-assemble into thermodynamically-stable "vertex up" honeycomb, square, and hexagonal lattices. Crucial for these findings was the development of a numerical method [68] that takes into account interfacial deformations. This method allows to go beyond the so-called Pieranski approximation [12] which assumes the fluid-fluid interface to be flat even when a particle is adsorbed. Although often used in the literature [109-113], Pieranski's approximation can lead to erroneous predictions, and in the case of cubes it cannot 


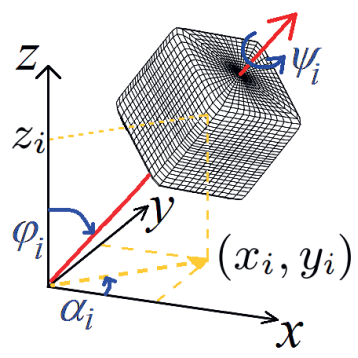

Figure 3.1: Sketch of the single-particle configuration $\left(x_{i}, y_{i}, z_{i}, \varphi_{i}, \psi_{i}, \alpha_{i}\right)$ of the generic $i$-th (smoothedge) cube adsorbed at the fluid-fluid interface, with $i=1, \ldots N$ and $N$ the total number of adsorbed cubes. The $i$-th cube has a center of mass with Cartesian coordinates $x_{i}, y_{i}, z_{i}$, a polar angle $\varphi_{i}$ between its vertical axis (sketched with a red arrow) and the $z$ axis, an internal Euler angle $\psi_{i}$ around its vertical axis, and an azimuthal angle $\alpha_{i}$ in the $z=0$ plane. The plane $z=0$ corresponds to the flat fluid-fluid interface when no particle is adsorbed.

predict the equilibrium hexapolar-generating "vertex up" configuration when $\theta=\pi / 2[33]$.

Despite the importance that linear aggregates of cubes trapped at a fluid-fluid interface appear to have in technological applications [31,32], no theoretical investigation on this kind of aggregates has been performed so far. To fill this gap, we extend in this Chapter the theoretical calculations of Refs. $[33,34]$ in order to understand under what conditions linear chains of cubes could be more stable than other $2 \mathrm{D}$ structures. To this aim we first study the deformation field induced by a single adsorbed cube as a function of the Young contact angle $\theta$ and of the particle configuration. We find that, below $\theta=\pi / 2$, a regime of contact angles exists in which the speculation of Refs. [31,32] concerning a quadrupolar deformation field induced by a particle adsorbed in the "edge up" configuration is correct. Then, by computing the capillary interactions acting among a 2D lattice of $N \rightarrow \infty$ cubes, we find that in this regime the quadrupolar deformation field leads to the formation of stable linear aggregates which replace the hexagonal patterns observed at $\theta=\pi / 2$. Besides the linear chains we also find stable "edge up" square structures. However, for $\theta$ below a certain threshold, we find that both the linear and "edge up" square structures become metastable with respect to close-packed square aggregates, in line with the experimental findings. Nevertheless, in contrast to speculations in Refs. [31,32], the stabilization of the close-packed square aggregates appears to be driven by packing entropy rather than by a monopolar deformation field. Finally, we introduce a simple free-energy model to estimate the interplay between capillary interactions and particle entropy. In this way we aim to include in our analysis the case of nm-sized particles for which, in contrast with $\mu \mathrm{m}$-sized particles, entropic effects cannot be neglected. We summarize our results in a series of temperature-density or size-density phase diagrams.

This Chapter is organized as follows: after briefly reviewing our numerical method in Section 3.2 , we report our results in Section 3.3, and present our conclusions and a discussion in Section 3.4

\section{$3.2 \quad$ Numerical method}

In this Section, we briefly review the numerical method developed in Ref. [68]. We refer to Ref. [69] for more details, and to Refs. [30,33,34,115-117] for other examples of its application 
to colloidal particles at fluid-fluid interfaces and to a droplet in contact with heterogeneous surfaces.

We consider two immiscible, homogeneous, and incompressible fluids, separated by a fluidfluid interface of surface tension $\gamma$. We introduce a Cartesian coordinate system $x, y, z$, and by convention call fluid 1 and fluid 2 the fluid at $z \rightarrow+\infty$ and $z \rightarrow-\infty$, respectively. We assume the fluid-fluid interface as a 2D, possibly curved surface with zero thickness, coinciding with a plane parallel to $z=0$ when no particles are adsorbed. In the case that colloidal particles are adsorbed, the equilibrium shape of the fluid-fluid interface is given by the Young-Laplace equation, with Young's law as a boundary condition for the Young contact angle $\theta$ [68]. This is the angle formed by the fluid-fluid interface along the three-phase contact line, i.e. where the fluid-fluid interface encounters the solid surface of the adsorbed particles. Calling $\gamma_{1}$ and $\gamma_{2}$ the solid-fluid surface tension of the particles with fluid 1 and fluid 2, respectively, Young's law states that $\theta$ (measured inside fluid 2) is given by $\cos \theta=\left(\gamma_{1}-\gamma_{2}\right) / \gamma$.

In our formalism we rewrite the force balance described by the Young-Laplace equation as a minimum condition of a suitable (free) energy, and perform the minimization numerically. To this aim we represent the fluid-fluid interface as a grid of points, and the fixed positions and orientations of $N$ adsorbed particles by a $6 N$-dimensional vector $\boldsymbol{\Omega}=\left(\boldsymbol{\Omega}_{1}, \ldots, \boldsymbol{\Omega}_{N}\right)$, with $\boldsymbol{\Omega}_{i}=\left(x_{i}, y_{i}, z_{i}, \varphi_{i}, \psi_{i}, \alpha_{i}\right)$ defining the configuration of the $i$-th particle $(i=1, \ldots, N)$. As shown in Fig. 3.1, we consider smooth-edge cubic particles in this Chapter. The in-plane Cartesian coordinates of the center of mass of the $i$-th particle is denoted by $x_{i}$ and $y_{i}, z_{i}$ denotes the difference between the Cartesian coordinate $z$ of the center of mass and the interface height far away from the particle, $\varphi_{i}$ the polar angle of the vertical axis with respect to the far-field interface normal, $\psi_{i}$ the angle of the particle around its vertical axis, and $\alpha_{i}$ is the azimuthal angle of the vertical axis in the interface plane. Moreover we write the free energy $E_{N}$ of a system of $N$ colloidal particles adsorbed at a fluid-fluid interface as [68]

$$
E_{N}(\boldsymbol{\Omega})=\gamma(S(\boldsymbol{\Omega})-A+W(\boldsymbol{\Omega}) \cos \theta)
$$

where $S(\boldsymbol{\Omega})$ is the total fluid-fluid surface area, $W(\boldsymbol{\Omega})$ is the total surface area between fluid 1 and the particles, and the constant $A$ is the planar fluid-fluid surface area when no particle is adsorbed, and is included in Eq. (3.1) to set the energy level $E_{N}=0$ when all particles are desorbed from the interface and are immersed in fluid 2. Finally we use $L$ and $\Sigma \sim 5.65 L^{2}$ to indicate the side and the surface area of each particle, respectively, where $\Sigma \neq 6 L^{2}$ because the cubes are slighlty rounded. Note that the input parameters $\theta$ and $\gamma$ characterise the two fluids and the material of the particles, and that $\gamma$ is a mere scaling factor of the energy $E_{N}$ at this point.

We now adopt the following protocol: first we fix the contact angle $\theta$. Then, by varying $\Omega$ to minimize $E_{N}$, we obtain the equilibrium particle configuration and the corresponding shape of the fluid-fluid interface, at fixed contact angle $\theta$. We repeat this protocol for several $\theta$ in order to investigate different particle wettabilities. However, it is important to note that in this Chapter we are interested in the deformation fields induced by equilibrium configurations as well as by (metastable) non-equilibrium ones.

\subsection{Results}

In this Section we investigate whether we can find conditions for colloidal cubes to assemble into stable linear chains rather than into other 2D structures, like square, hexagonal, or honeycomb 


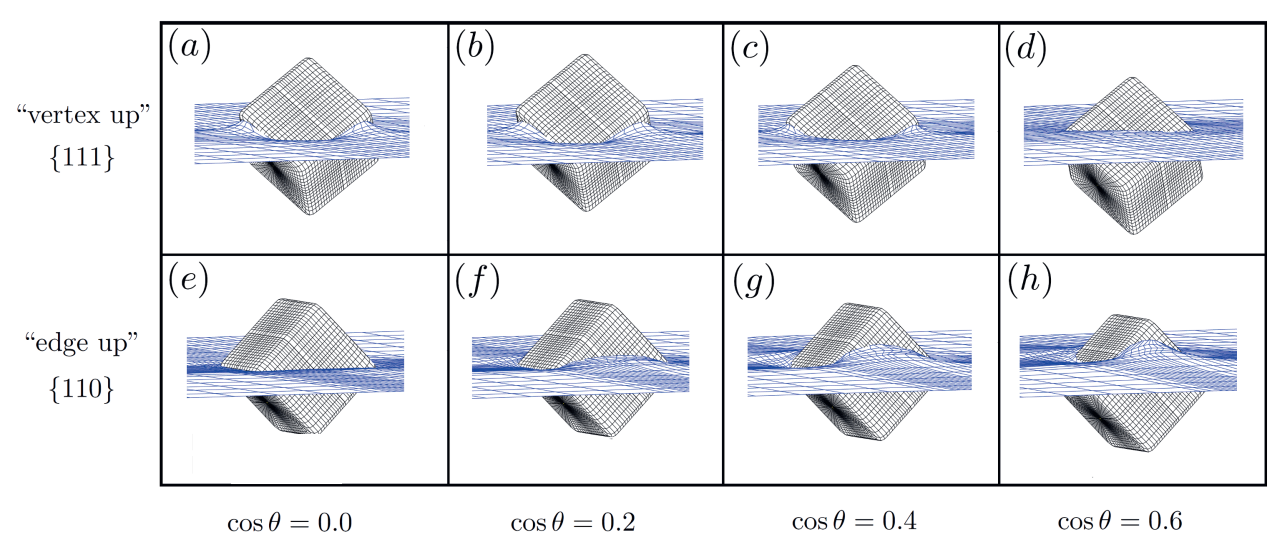

Figure 3.2: 3D plot of the deformation field induced by a single cube of side length $L$ adsorbed at a fluid-fluid interface in the "vertex up" $\{111\}$ and "edge up" $\{110\}$ configuration, respectively, for several Young contact angles $\theta$. For $\cos \theta=0$ (no preference for either of the two fluids), a cube adsorbed in the $\{111\}$ configuration $(a)$ generates a hexapolar deformation field, while a cube adsorbed in the $\{110\}$ configuration $(e)$ does not induce any deformation. By increasing $\cos \theta$ (such that the cube favours the lower fluid) the intensity of the hexapolar deformation field of the $\{111\}$ configuration decreases $((b)$ and $(c))$ until it essentially vanishes in $(d)$ where $\cos \theta=0.6$, while the flat interface of the $\{110\}$ configuration evolves into a quadrupolar deformation field whose intensity increases with $\cos \theta((f)$ through $(h))$.

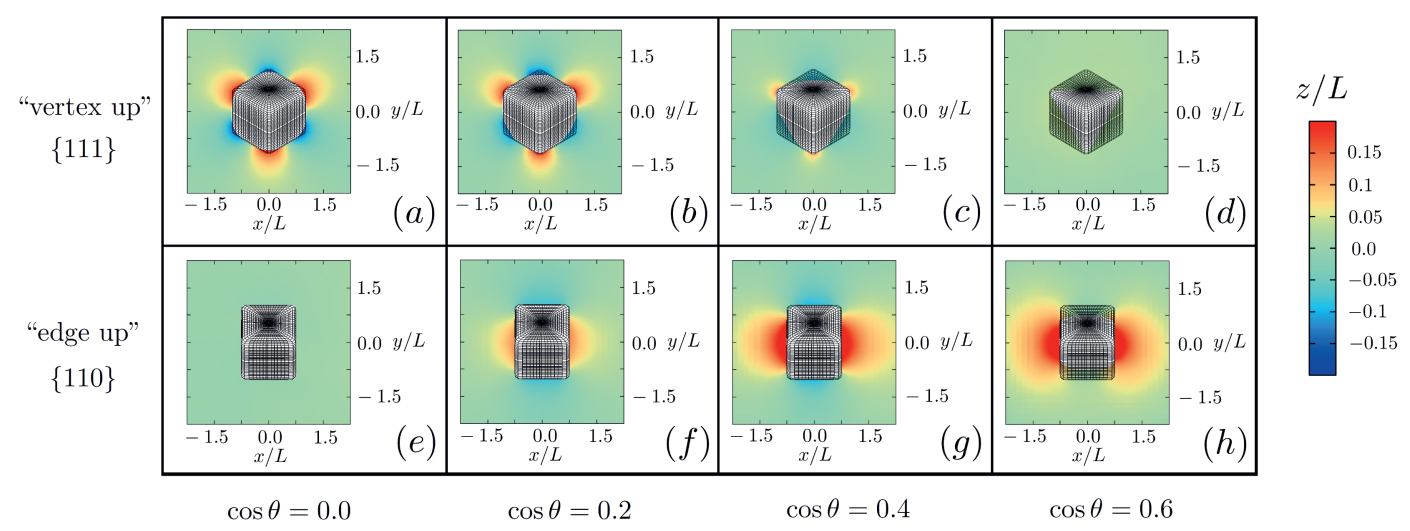

Figure 3.3: Contour plot of the deformation field induced by a single cube of side length $L$ adsorbed at a fluid-fluid interface in the "vertex up" $\{111\}$ and "edge up" $\{110\}$ configuration, respectively, for the same Young contact angles $\theta$ as in Fig. 3.2. 


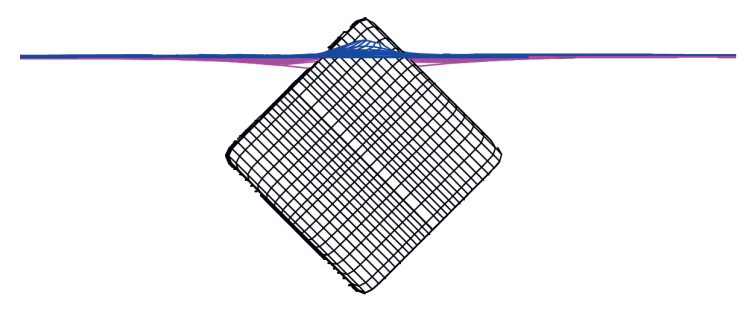

Figure 3.4: Quadrupolar deformation field induced by a cubic particle with a Young contact angle $\cos \theta=0.6$, adsorbed at a fluid-fluid interface in the "edge up" $\{110\}$ configuration.

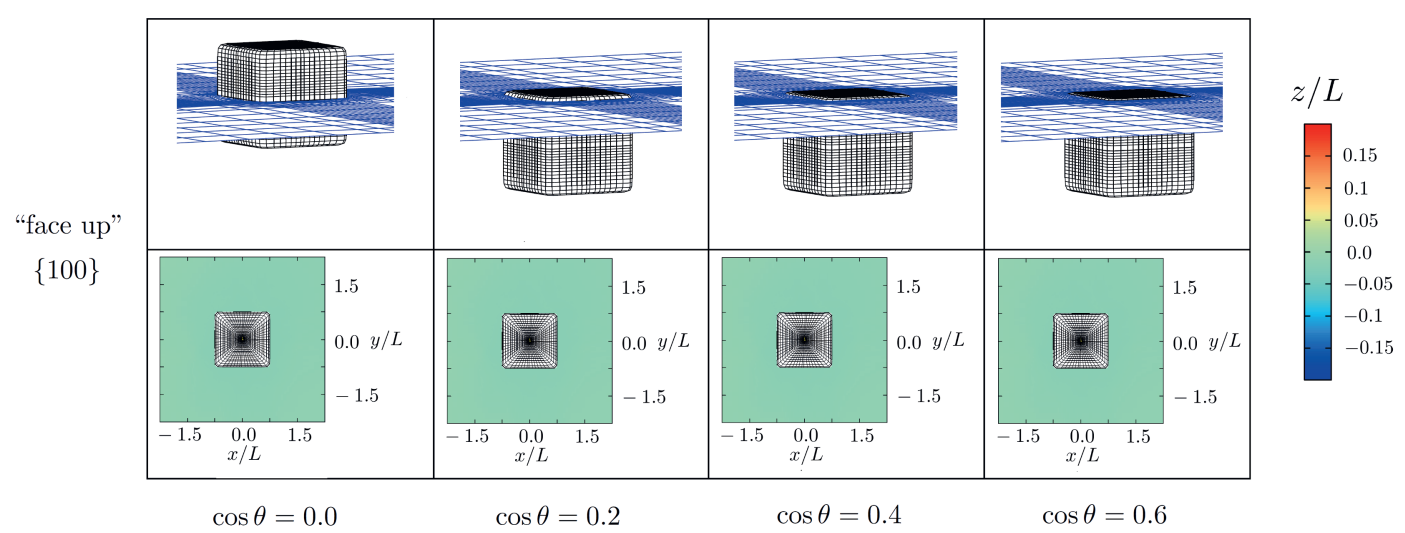

Figure 3.5: Deformation field induced by a single cube of side $L$ adsorbed at a fluid-fluid interface in the "face up" $\{100\}$ configuration, for several Young contact angles $\theta$. The particle in this configuration never deforms the interface. 


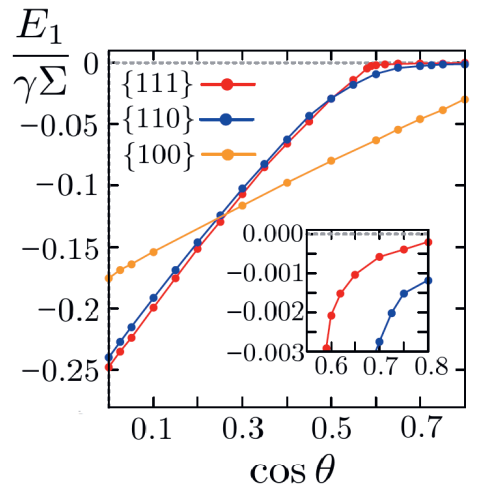

Figure 3.6: Adsorption energies $E_{1}^{\{111\}}, E_{1}^{\{110\}}$ and $E_{1}^{\{100\}}$ of a single cube in the $\{111\},\{110\}$ and $\{100\}$ configuration, respectively, as a function of the Young contact angle $\theta$. The energies are expressed in units of $\gamma \Sigma$, with $\gamma$ the fluid-fluid surface tension and $\Sigma$ the surface area of the particle. The inset shows a zoom of the graph in the region $\cos \theta \geq 0.55$, where $E_{1}^{\{100\}} \ll E_{1}^{\{110\}}<E_{1}^{\{111\}}$; for $\cos \theta \leq 0.25$, the $\{111\}$ configuration has the lowest free energy.

lattices. We first study the deformation field induced by a single adsorbed cube as a function of the Young contact angle $\theta$ and of the particle configuration. Then we numerically compute the capillary interactions acting among a 2 D lattice of $N \rightarrow \infty$ cubes adsorbed at an interface and perform a stability analysis among the several phases that the cubes can form. Finally, by using a simple free-energy model, we summarize our results in a series of density-temperature phase diagrams. Here the temperature is actually the dimensionless combination $k_{\mathrm{B}} T /(\gamma \Sigma)$, which in practise is seen as an inverse particle size.

Following Refs. [33,34] we will refer to the "vertex up", "edge up", and "face up" configurations, as the $\{111\},\{110\}$, and $\{100\}$ configurations, respectively.

\subsubsection{Hexapolar and quadrupolar deformation fields}

As stated in the Introduction, in Refs. [31,32] it has been suggested that the self-assembly of micronscale cubes trapped at a fluid-fluid interface into hexagonal aggregates and linear ones would be caused by two different kinds of deformation fields induced by the adsorbed particles. More specifically, the stabilization of the hexagonal phase would be caused by a hexapolar deformation field generated by cubes in the $\{111\}$ configuration while the stabilization of the linear phase would be caused by a quadrupolar deformation field generated by cubes in the $\{110\}$ configuration. While these suggestions agree with the theoretical calculations performed so far in the former case, they disagree in the latter case. Indeed, in Refs. [33,34] it has been theoretically predicted that the deformation field induced by a cube adsorbed in the $\{111\}$ configuration is dominated by the hexapolar mode, but it has also been found that the same cube adsorbed in the $\{110\}$ configuration does not induce any interfacial deformation. However, these calculations have been performed only for cubes with contact angle $\theta=\pi / 2$. Since the wettability has been experimentally proven to play a decisive role in the stabilization of the various phases at the interface, we here extend the calculations performed in Refs. [33, 34] of the deformation field induced by a single cube adsorbed at a fluid-fluid interface, from the case $\theta=\pi / 2$ to smaller values of $\theta$. 
As explained in Section 3.2, the configuration of a single adsorbed cube at the interface, is given by $\Omega=(x, y, z, \varphi, \psi, \alpha)$, where the subscript " 1 " can be omitted since we consider only a single particle (i.e. $N=1$ ), and the equilibrium shape of the fluid-fluid interface is the one that minimizes the free energy $E_{1}$ (Eq. (3.1)), for fixed values of $\Omega$ and $\theta$. We note that symmetry considerations in the case of a single particle show that the energy $E_{1}$ is independent of $x, y$, and $\alpha$, such that the particle configuration is specified by only $\varphi, \psi$, and $z$. Furthermore the equilibrium height $z$ of the cube at the interface is automatically found by our numerical method, for each fixed $\varphi, \psi$, and $\theta$, by allowing the two fluids to exchange volume while minimizing $E_{1}[30]$. The $\{111\}$ configuration is specified by $\psi_{\{111\}}=\pi / 4, \varphi_{\{111\}}=\pi / 3.29$, while the $\{110\}$ configuration by $\psi_{\{110\}}=0, \varphi_{\{110\}}=\pi / 4$. Note that we consider $\varphi_{\{111\}}=\pi / 3.29$ rather than $\varphi_{\{111\}}=\pi / 4$ since the former configuration has a (slightly) lower energy than the latter one $[33,34]$. In the following we will indicate the energies of a single cube adsorbed in the $\{111\}$ and $\{110\}$ configurations with $E_{1}^{\{111\}}$ and $E_{1}^{\{110\}}$, respectively.

The results of the minimization procedure for $\cos \theta \in\{0,0.2,0.4,0.6\}$ are shown in Figs. 3.2 and 3.3. We find that the deformation field induced by the adsorption process strongly depends on the contact angle $\theta$. In agreement with Refs. [33,34], we find that for $\cos \theta=0$ a cube adsorbed in the $\{111\}$ configuration generates a hexapolar deformation field consisting of three rises and three depressions, exhibiting a 3-fold symmetry around the particle (see Figs. 3.2(a) and 3.3(a)), while the same particle adsorbed in the $\{110\}$ configuration does not induce any deformation (see Figs. 3.2(e) and 3.3(e)). The situation changes by changing the contact angle. By decreasing $\theta$, the intensity of the hexapolar deformation field decreases until it essentially vanishes for $\cos \theta=0.6$ (see Figs. $3.2(d)$ and $3.3(d)$ ). In this case the fluid-fluid interface becomes actually flat. By contrast, the flat interface observed for the $\{110\}$ configuration at $\cos \theta=0$ evolves into a quadrupolar deformation field consisting of two rises and two depressions, showing a 2-fold symmetry around the particle, for larger $\cos \theta$. In particular the intensity of the quadrupole increases with increasing $\cos \theta$. Note that the quadrupolar deformation field for $\cos \theta=0.6$ has two depressions that are more spread and less intense than the two rises. As a consequence the depressions can hardly be appreciated in the contour plot of Fig. 3.3(h). Nevertheless the presence of the quadrupole at $\cos \theta=0.6$ can clearly be seen in the 3D plot of Fig. 3.4.

In addition to the deformation fields induced by a single cube adsorbed in the $\{111\}$ and $\{110\}$ configurations, we employ our numerical method to compute the deformation field induced by a single cube adsorbed in the $\{100\}$ configuration $\left(\psi_{\{100\}}=0, \varphi_{\{100\}}=0\right)$, for several values of the contact angle $\theta$. We indicate the energy of a single cube adsorbed in this configuration with $E_{1}^{\{100\}}$. As shown in Fig. 3.5, we find that in these cases the cube never deforms the interface. This finding disagrees with the speculation of Refs. [31,32], where it was suggested that cubes with $30 \mu \mathrm{m}$ edge length and a very small contact angle $\theta \sim 0$ would induce a monopolar deformation field at the interface, when adsorbed in the $\{100\}$ configuration. This does not appear to be the case. Indeed, by considering typical values for the mass density difference between the particle and the fluids $\delta \sim 10^{3} \mathrm{~kg} \mathrm{~m}^{-3}$, the fluid-fluid surface tension $\gamma \sim 10^{-2} \mathrm{~N} \mathrm{~m}^{-1}$, and (the modulus of) the gravitational acceleration $g$ on planet Earth, we obtain a Bond number Bo $\equiv(L / \sqrt{\gamma /(g \delta)})^{2} \ll 1$ for a cubic particle with side length $L \ll 1 \mathrm{~mm}$. It follows that a monopolar mode is not expected for a sub-mm sized particle at its equilibrium adsorption configuration, unless non-gravitational external forces act on the particle.

We plot $E_{1}^{\{111\}}, E_{1}^{\{110\}}$, and $E_{1}^{\{100\}}$ as a function of the contact angle $\theta$ in Fig. 3.6. We observe that the particle configurations discussed above are not always equilibrium configu- 


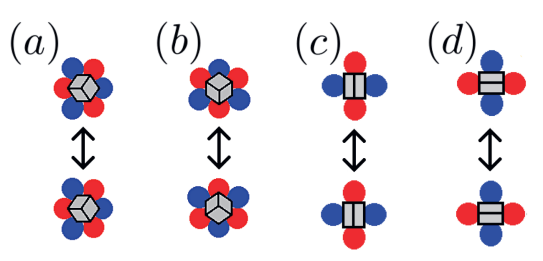

Figure 3.7: Energetically-favorable capillary bonds induced by $N=2$ cubes adsorbed at a fluidfluid interface in the $\{111\}((a)-(b))$, and $\{110\}((c)-(d))$ configuration, respectively, where red denotes a rise and blue a depression. (a) Dipole-dipole bond, when a rise-depression dipole of one hexapole overlaps with a rise-depression dipole of another hexapole. (b) Tripole-tripole bond, when a depression-rise-depression tripole of one hexapole overlaps with a depression-rise-depression tripole of the other hexapole. (c) Monopole-monopole bond, when a rise monopole of one quadrupole overlaps with a rise monopole of the other quadrupole. $(d)$ Monopole-monopole bond, when a depression monopole of one quadrupole overlaps with a depression monopole of the other quadrupole.

rations. Indeed, the minimal single-particle adsorption energy is $E_{1}^{\{111\}}$ for $\cos \theta \leq 0.25$ and $E_{1}^{\{100\}}$ for $\cos \theta>0.25$. It follows that the equilibrium (minimal (free) energy) configuration of a single adsorbed cube is the $\{111\}$ in the former case, and the $\{100\}$ in the latter case. One could therefore naively think that the linear-chain phase will never be stable at an interface since the quadrupolar-generating $\{110\}$ single-particle configuration is always metastable with respect to the $\{111\}$ and the $\{100\}$ ones. However, this is not necessarily true because when more particles are adsorbed at an interface, capillary interactions have to be considered such that an equilibrium phase of many particles can be stabilized even though the corresponding single-particle configuration is not the stable one. In particular we will see that a regime exists for $\cos \theta \geq 0.35$ in which the quadrupolar and the hexapolar deformation fields induced by cubes in the $\{110\}$ and $\{111\}$ configurations, respectively, have comparable intensities. As we will show in the next subsection it is in this regime that the linear-chain phase of $\{110\}$ particles is stable, even though the single-particle equilibrium configuration is the $\{100\}$ one in this regime of contact angles $\theta$.

Furthermore we observe that for $\cos \theta \geq 0.55, E_{1}^{\{100\}} \ll E_{1}^{\{110\}}<E_{1}^{\{111\}}$, with $E_{1}^{\{110\}}$ and $E_{1}^{\{111\}}$ approaching zero. In other words the adsorption energies of cubes in the $\{111\}$ and $\{110\}$ configuration are very weak in this regime of contact angles and, as we will show, this results in weak capillary interactions.

\subsubsection{Stable chains of cubes at a fluid-fluid interface}

Two or more cubes adsorbed in the hexapolar-generating $\{111\}$ and in the quadrupolargenerating $\{110\}$ configurations give rise to different kinds of energetically-favorable bonds. This can be understood by considering that a pair of cubes $(N=2)$ adsorbed at a fluid-fluid interface can attract or repel each other, depending on their relative azimuthal orientation $\left|\alpha_{1}-\alpha_{2}\right|$. Attractive relative orientations are those that allow overlap of interfacial deformations with the same sign, i.e. rises with other rises and depressions with other depressions. In this case the fluid-fluid surface area, and hence the energy $E_{2}$ (Eq. (3.1)), decrease as the particles approach each other. Repulsive relative orientations, instead, are those in which interfacial deformations with the opposite sign, i.e. rises with depressions, overlap. In this case, the fluid-fluid surface area, and hence the energy $E_{2}$ (Eq. (3.1)), increase as the particles approach 


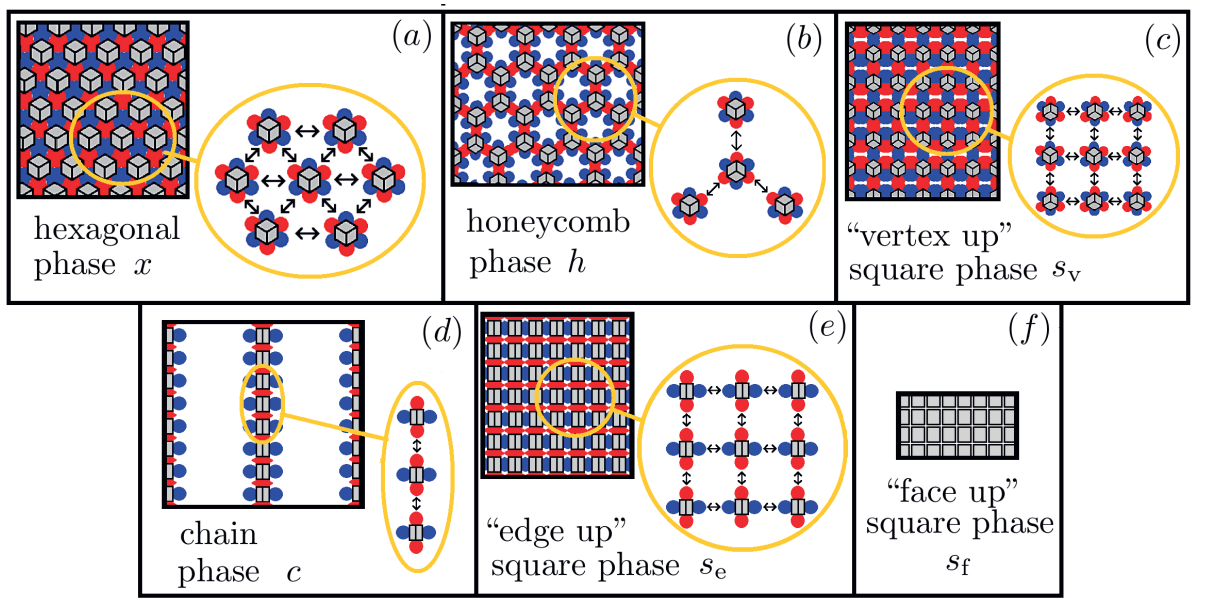

Figure 3.8: Phases formed by a $2 \mathrm{D}$ lattice of $N \rightarrow \infty$ colloidal cubes adsorbed at a fluid-fluid interface with schematic capillary rises (red) and depressions (blue). Cubes adsorbed in the hexapolargenerating $\{111\}$ configuration can give rise to hexagonal $x(a)$, honeycomb $h(b)$, and "vertex up" square $s_{\mathrm{v}}(c)$ phases, while cubes adsorbed in the quadrupolar-generating $\{110\}$ configuration can give rise to chains $c(d)$, and "edge up" square $s_{\mathrm{e}}(e)$ phases. No capillary interactions act among cubes in the $\{100\}$ configuration, as cubes in this configuration do not deform the interface. The formation of a "face up" square $s_{\mathrm{f}}$ phase $(f)$ would then be driven by packing entropy.

each other.

As already explained in Refs. [33,34] and as shown in Fig. 3.7, it follows that $N=2$ cubes adsorbed in the $\{111\}$ configuration can form two different kinds of energetically-favorable bonds: a dipole-dipole bond, when a rise-depression dipole of one hexapole overlaps with a rise-depression dipole of the other hexapole (see Fig. 3.7( $a)$ ), and a tripole-tripole bond, when either a rise-depression-rise or a depression-rise-depression tripole of one hexapole overlaps with, respectively, a rise-depression-rise or a depression-rise-depression tripole of the other hexapole (see Fig 3.7(b)). By contrast, two cubes adsorbed in the $\{110\}$ configuration can only form a monopole-monopole bond, when a rise monopole of one quadrupole overlaps with a rise monopole of the other quadrupole (see Fig. 3.7(c)), or when a depression monopole of one quadrupole overlaps with a depression monopole of the other quadrupole (see Fig. 3.7 $(d)$ ).

The different energetically-favorable bonds induced by cubes in the $\{111\}$ and $\{110\}$ configuration, respectively, lead in turn to the formation of different phases at the interface, as illustrated in Fig. 3.8. In particular, a regular lattice of $N \rightarrow \infty$ cubes adsorbed in the $\{111\}$ configuration can lead to the formation of three distinct $2 \mathrm{D}$ phases, namely hexagonal $(x)$, honeycomb $(h)$, and "vertex up" square $\left(s_{\mathrm{v}}\right)$ phases. In the $x$ phase each particle forms a dipole-dipole bond with each of its six nearest neighbors (see Fig. 3.8(a)), in the $h$ phase each particle forms a tripole-tripole bond with each of its three nearest neighbors (see Fig. 3.8(b)), in the $s_{\mathrm{v}}$ phase each particle forms a dipole-dipole bond with two opposite nearest neighbors and a tripole-tripole bond with the two remaining neighbors (see Fig. 3.8(c)). By constrast, $N \rightarrow \infty$ cubes adsorbed in the $\{110\}$ configuration can lead to the formation of two distinct phases, namely chains $(c)$, and "edge up" square $\left(s_{\mathrm{e}}\right)$ phases. In the $c$ phase each particle forms a monopole-monopole bond with only two neighbors, along a single linear "preferred" direction 

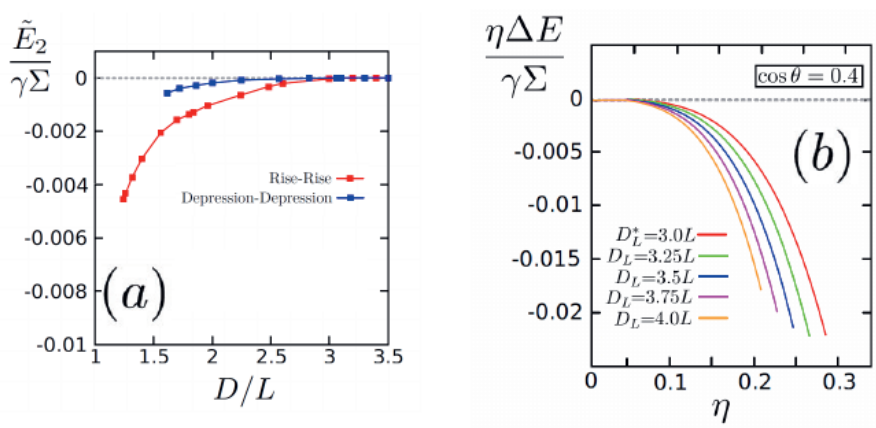

Figure 3.9: (a) Interaction energy per particle $\tilde{E}_{2} \equiv E_{2} / 2-E_{1}$ of two cubes with side $L$ and a Young contact angle $\cos \theta=0.2$ adsorbed in the $\{110\}$ configuration, as a function of the distance $D$ between their centers of mass. In red the case of cubes with two overlapping rises is depicted, in blue the case of cubes with two overlapping depressions. The energy $\tilde{E}_{2}$ is plotted in units of $\gamma \Sigma$, with $\gamma$ the fluid-fluid surface tension and $\Sigma$ the surface area of the particle. $E_{1}$ and $E_{2}$ are given by Eq. (3.1) of Section 3.2, in the case $N=1$ and $N=2$, respectively. (b) Dimensionless (capillary) interaction energy per unit area $\eta \Delta E /(\gamma \Sigma)$ in the $c$ phase as a function of the particle packing fraction $\eta$, for $\cos \theta=0.4 . \Delta E \equiv E_{N} / N-E_{1}^{\{110\}}$ is the (capillary) interaction energy per particle minus the adsorption energy of a single particle in the $\{110\}$ configuration, and is expressed in units of $\gamma \Sigma$, where $\gamma$ is the fluid-fluid surface tension and $\Sigma$ is the surface area of a particle. With the different colours we indicate $\eta \Delta E /(\gamma \Sigma)$ computed for several values of the lateral distance $D_{L}$ among the chains (see Fig. 3.14 and Section 3.3.2). $D_{L}^{*}=3 L$ (with $L$ denoting the side of the cube) corresponds to the smallest distance for which two cubes in the $\{110\}$ configurations do not interact. We observe that considering $D_{L}>D_{L}^{*}$ does not significantly affect the $\eta$ dependence of $\eta \Delta E$, unless $D_{L}$ is too large. 

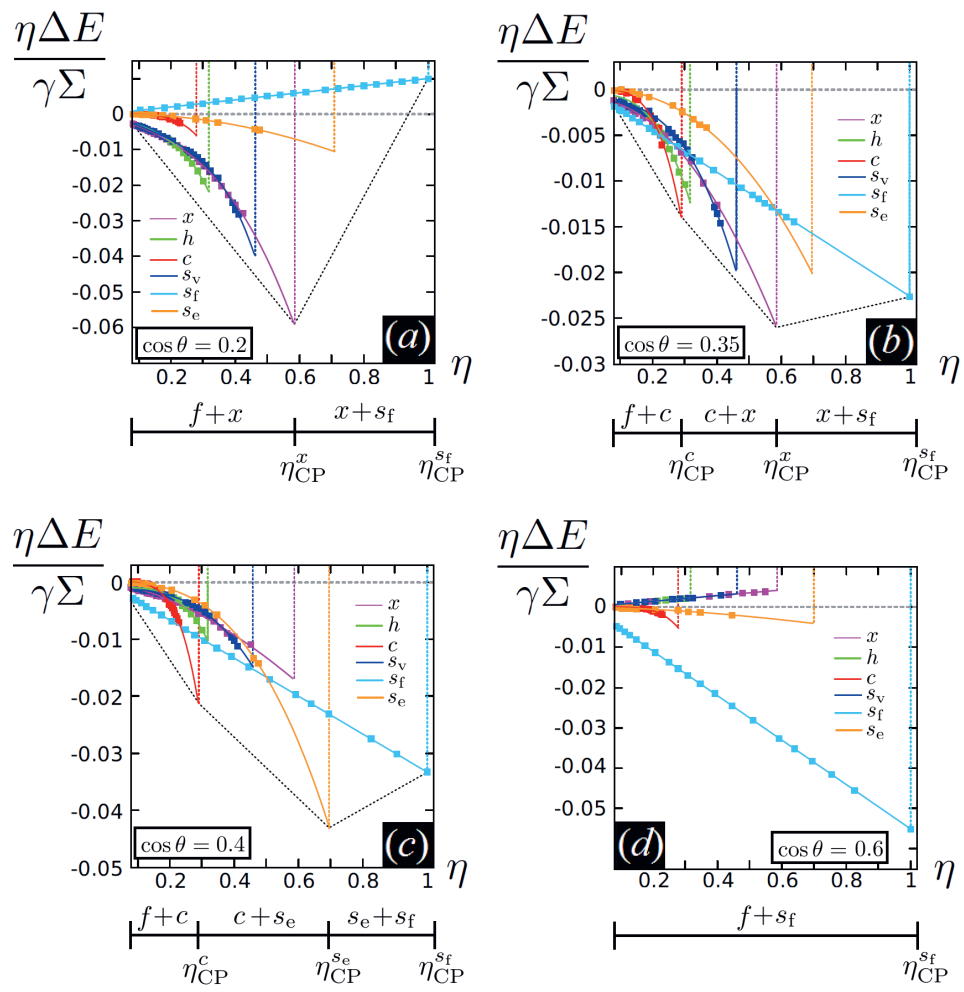

Figure 3.10: Dimensionless (capillary) interaction energy per unit area $\eta \Delta E /(\gamma \Sigma)$ as a function of the particle packing fraction $\eta$, for $\cos \theta \in\{0.2,0.35,0.4,0.6\} \cdot \eta_{\mathrm{CP}}^{\xi}$ indicates the closest-packing of the $\xi=x, c, s_{\mathrm{e}}, s_{\mathrm{f}}$ phases. $\Delta E \equiv E_{N} / N-E_{1}^{\{110\}}$ is the (capillary) interaction energy per particle minus the adsorption energy of a single particle in the $\{110\}$ configuration, and is expressed in units of $\gamma \Sigma$, where $\gamma$ is the fluid-fluid interfacial tension and $\Sigma$ is the surface area of a particle. We show results for the phase with chains $c$ (red), the hexagonal phase $x$ (magenta), the "face up" square phase $s_{\mathrm{f}}$ (light-blue), the "edge up" square phase $s_{\mathrm{e}}$ (yellow), the "vertex up" square phase $s_{\mathrm{v}}$ (blue), and the honeycomb phase $h($ green). Dotted black lines indicate the common tangent construction. At $\cos \theta=0.2$ (see (a)) an extremely dilute disordered fluid phase $f$ coexists with the close-packed $x$ phase for $\eta<\eta_{\mathrm{CP}}^{x}$, while for $\eta>\eta_{\mathrm{CP}}^{x}$ the close-packed $x$ phase coexists with the close-packed $s_{\mathrm{f}}$ phase. At $\cos \theta=0.35$ (see $(b)$ ), for $\eta<\eta_{\mathrm{CP}}^{c}$ the $f$ phase coexists with the close-packed $c$ phase, for $\eta_{\mathrm{CP}}^{c}<\eta<\eta_{\mathrm{CP}}^{x}$ the close-packed $c$ phase coexists with the close-packed $x$ phase, while for $\eta>\eta_{\mathrm{CP}}^{x}$ the $x$ phase coexists with the close-packed $s_{\mathrm{f}}$ phase. Instead, at $\cos \theta=0.4$ (see $\left.(c)\right)$ for $\eta<\eta_{\mathrm{CP}}^{c}$ the $f$ phase coexists with the close-packed $c$ phase, for $\eta_{\mathrm{CP}}^{c}<\eta<\eta_{\mathrm{CP}}^{s_{\mathrm{e}}}$ the close-packed $c$ phase coexists with the close-packed $s_{\mathrm{e}}$ phase, while for $\eta>\eta_{\mathrm{CP}}^{s_{\mathrm{e}}}$ the $s_{\mathrm{e}}$ phase coexists with the close-packed $s_{\mathrm{f}}$ phase. Finally, at $\cos \theta=0.6$ (see $(d))$ the $s_{\mathrm{f}}$ phase is the only stable phase, besides the $f$ phase. 


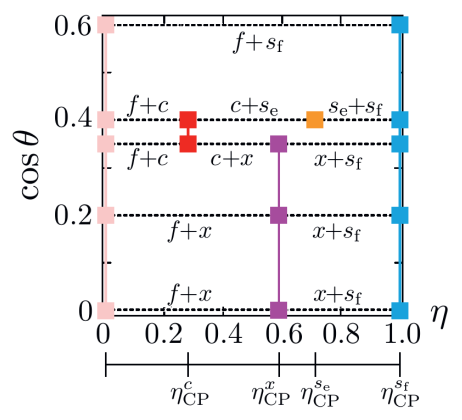

Figure 3.11: Ground state $(T=0)$ phase diagram reporting the particle packing fraction $\eta$ on the horizontal axis, and the Young contact angle $\theta$ on the vertical axis. With light-pink we represent the dilute fluid phase $f$, with magenta the hexagonal phase $x$, with red the phase with linear chains $c$, with yellow the "edge up" square phase $s_{\mathrm{e}}$, and with light-blue the "face up" square phase $s_{\mathrm{f}} . \eta_{\mathrm{CP}}^{\xi}$ indicates the closest-packing of $\xi=x, c, s_{\mathrm{e}}, s_{\mathrm{f}}$. Dashed lines indicate regions of phase coexistence.

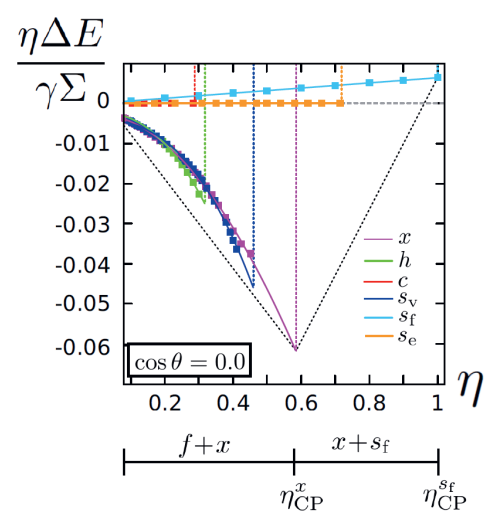

Figure 3.12: Dimensionless (capillary) interaction energy per unit area $\eta \Delta E /(\gamma \Sigma)$ as a function of the particle packing fraction $\eta$, for $\cos \theta=0.0 . \eta_{\mathrm{CP}}^{\xi}$ indicates the closest-packing of $\xi=x, s_{\mathrm{f}}$. $\Delta E \equiv E_{N} / N-E_{1}^{\{110\}}$ is the (capillary) interaction energy per particle minus the adsorption energy of a single particle in the $\{110\}$ configuration, and is expressed in units of $\gamma \Sigma$, where $\gamma$ is the fluid-fluid surface tension and $\Sigma$ is the surface area of a particle. We show results for the phase with chains $c$ (red), the hexagonal phase $x$ (magenta), the "face up" square phase $s_{\mathrm{f}}$ (light-blue), the "edge up" square phase $s_{\mathrm{e}}$ (yellow), the "vertex up" square phase $s_{\mathrm{v}}($ blue), and the honeycomb phase $h$ (green). Dotted black lines indicate the common tangent construction. We see that an extremely dilute disordered fluid phase $f$ coexists with the close-packed $x$ phase for $\eta<\eta_{\mathrm{CP}}^{x}$, while for $\eta>\eta_{\mathrm{CP}}^{x}$ the close-packed $x$ phase coexists with the close-packed $s_{\mathrm{f}}$ phase. 

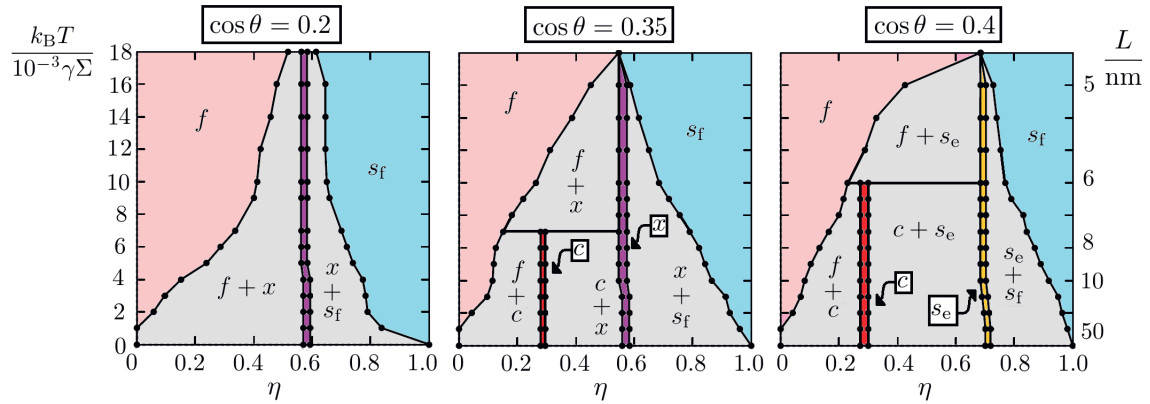

Figure 3.13: Temperature-density phase diagram for $\cos \theta \in\{0.2,0.35,0.4\}$. The left vertical axis is the dimensionless temperature $k_{\mathrm{B}} T /(\gamma \Sigma)$, with $\gamma$ the fluid-fluid surface tension, $\Sigma$ the total surface area of a particle, $k_{\mathrm{B}}$ the Boltzmann constant, and $T$ the temperature. The right vertical axis represents the corresponding value of the particle size $L$ at room temperature $(300 K)$ and using a typical surface tension $\gamma=0.01 \mathrm{~N} \mathrm{~m}^{-1}$. The horizontal axis is the particle density $\eta$, which is equal to 1 for the close-packed "face up" square phase $s_{\mathrm{f}}$. The colored areas indicate stable $2 \mathrm{D}$ one-phase regions at the fluid-fluid interface: light-pink indicates a disordered fluid phase $f$, while red, magenta, yellow, and light-blue the chains $c$, hexagonal $x$, "edge up" square $s_{\mathrm{e}}$, and "face up" square $s_{\mathrm{f}}$ phases, respectively. The gray areas indicate coexistence between two phases of different $\eta$, and the horizontal lines connect three phases coexisting at the $f-c-x$, and $f-c-s_{\mathrm{e}}$ triple points, respectively.

(see Fig. 3.8(d)), where the particles do not interact along the direction perpendicular to the principal one. In the $s_{\mathrm{e}}$ phase, each particle forms a monopole-monopole bond with four nearest neighbors, i.e. the cubes not only interact along a "preferred" direction as in the case of the chains, but also perpendicularly to this (see Fig. 3.8(e)).

We find that in principle two different chain phases exist. In the first case the chains are obtained by overlapping rises with rises, in the second case by overlapping depressions with depressions. As shown in Fig. 3.9(a), we find that the pair interaction acting between two cubes with overlapping rises is much stronger than the pair interaction acting between two cubes with overlapping depressions. As a consequence it is more favorable for cubes to assemble into chains with overlapping rises rather than overlapping depressions, and we will only consider the former case throughout the paper.

Finally no capillary interactions act among $N \rightarrow \infty$ cubes adsorbed in the $\{100\}$ configuration, since cubes in this configuration do not deform the interface. The formation of a "face up" square phase $s_{\mathrm{f}}$ (see Fig. 3.8(f)) is then entropy-driven at sufficiently high areal densities.

The intensity of the (capillary) interaction energy $E_{N}$ acting among a 2D lattice of $N \rightarrow \infty$ cubes in the several phases indicated above changes with the contact angle $\theta$, and consequently different phases stabilize at different $\theta$. To show this, we compute $E_{N}$ for the various phases as a function of $\theta$. To this aim we apply the numerical method described in Section 3.2 to a lattice unit cell, and impose periodic boundary conditions (PBCs) to the interface height profile. The size of the cell determines the particle packing fraction $\eta$ in the lattice, while the shape of the unit cell and the PBCs specify the particular phase under considerations. Details on the lattice unit cells and the PBCs used to define the several phases sketched in Fig. 3.8 can be found in Appendix A.

We observe that while the $x, h, s_{\mathrm{v}}, s_{\mathrm{e}}$ and $s_{\mathrm{f}}$ phases are 2D phases, the $c$ phase is essentially a $1 \mathrm{D}$ phase. It follows that the definition of the $2 \mathrm{D}$ packing fraction $\eta$ is rather arbitrary in 
this latter case. In order to overcome this problem, we describe the $c$ phase as a "gas" of chains that do not interact laterally (see Fig. 3.8(d)). As explained in Fig. 3.14, to define the $c$ phase we choose one side of the unit lattice cell such that the lateral distance $D_{L}$ among the chains corresponds to the smallest distance $D_{L}^{*}$ for which two cubes in the $\{110\}$ configuration do not interact. The case $D_{L}<D_{L}^{*}$ corresponds to laterally interacting chains, i.e. the $s_{\mathrm{e}}$ phase. On the other hand, as shown in Fig. 3.9(b), considering $D_{L}>D_{L}^{*}$ does not significantly affect the $\eta$ dependence of the capillary interaction energy per unit area $\eta E_{N}$ in the $c$ phase, at sufficiently low $\eta$.

We present our results for $\cos \theta \in\{0.2,0.35,0.4,0.6\}$ in Fig. 3.10. The horizontal axis represents the dimensionless areal density $\eta=N L^{2} / A$, which we will call packing fraction for convenience below, even though $L^{2}$ is only (close to) the areal coverage of a cube in the $\{100\}$ configuration. We indicate the closest-packing of a specific phase with $\eta_{\mathrm{CP}}^{\xi}$, where $\xi=$ $x, h, s_{\mathrm{v}}, c, s_{\mathrm{e}}, s_{\mathrm{f}}$. As explained in Fig. 3.14 these correspond to $\eta_{\mathrm{CP}}^{x}=0.59, \eta_{\mathrm{CP}}^{h}=0.32, \eta_{\mathrm{CP}}^{s_{\mathrm{v}}}=$ $0.46, \eta_{\mathrm{CP}}^{c}=0.28, \eta_{\mathrm{CP}}^{s_{\mathrm{e}}}=0.71$, and $\eta_{\mathrm{CP}}^{s_{\mathrm{f}}}=1$. Note that the $s_{\mathrm{f}}$ phase is the densest-packed state. The vertical axis of Fig. 3.10 represents the dimensionless energy per unit area $\eta \Delta E /(\gamma \Sigma)$, where $\Delta E \equiv E_{N} / N-E_{1}^{\{110\}}$ is the capillary interaction energy per particle minus a conveniently but arbitrary constant that we set to the adsorption energy of a single particle in the $\{110\}$ configuration, for each of the phases described above. In Fig. 3.10 we employ the common tangent construction as represented by the convex envelope (dashed black line), to determine the ground state $(T=0)$ phase diagram.

We find several scenarios by changing $\theta$. At $\cos \theta=0.2$ (Fig. 3.10(a)) the capillary interaction energy among cubes adsorbed in the $\{111\}$ configuration, i.e. cubes in the $x, h$, and $s_{\mathrm{v}}$ phases, is much stronger (more negative) than the capillary interaction energy among cubes adsorbed in the $\{110\}$ configuration, i.e. cubes in the $c$ and $s_{\mathrm{e}}$ phases. As a consequence, we find that for $\eta<\eta_{\mathrm{CP}}^{x}$ an extremely dilute disordered fluid phase $f$ coexists with the close-packed $x$ phase, while for $\eta>\eta_{\mathrm{CP}}^{x}$ the close-packed $x$ phase coexists with the close-packed $s_{\mathrm{f}}$ phase. By increasing $\cos \theta$, instead, the intensity of the capillary interaction energy among cubes in the $\{110\}$ configuration becomes stronger while the capillary interaction energy among cubes in the $\{111\}$ configuration simultaneously becomes weaker. This leads to the scenarios observed at $\cos \theta=0.35$ and $\cos \theta=0.4$. At $\cos \theta=0.35$ (Fig. 3.10(b)), for $\eta<\eta_{\mathrm{CP}}^{c}$ the $f$ phase coexists with the close-packed $c$ phase, for $\eta_{\mathrm{CP}}^{c}<\eta<\eta_{\mathrm{CP}}^{x}$ the close-packed $c$ phase coexists with the close-packed $x$ phase, while for $\eta>\eta_{\mathrm{CP}}^{x}$ the $x$ phase coexists with the close-packed $s_{\mathrm{f}}$ phase. Instead, at $\cos \theta=0.4$ (Fig. $3.10(c)$ ) for $\eta<\eta_{\mathrm{CP}}^{c}$ the $f$ phase coexists with the close-packed $c$ phase, for $\eta_{\mathrm{CP}}^{c}<\eta<\eta_{\mathrm{CP}}^{s_{\mathrm{e}}}$ the close-packed $c$ phase coexists with the close-packed $s_{\mathrm{e}}$ phase, while for $\eta>\eta_{\mathrm{CP}}^{s_{\mathrm{e}}}$ the $s_{\mathrm{e}}$ phase coexists with the close-packed $s_{\mathrm{f}}$ phase. Finally, at $\cos \theta=0.6$ (Fig. $3.10(d))$ the $s_{\mathrm{f}}$ phase has the lowest energy per unit area $\eta \Delta E$ for each $\eta$, and the capillary interactions among cubes in the $\{111\}$ as well as in the $\{110\}$ configuration are very weak. It follows that at this contact angle the $s_{\mathrm{f}}$ phase is the only stable phase, besides the $f$ phase. Since in the $s_{\mathrm{f}}$ phase no capillary interactions act among the cubes, its stabilization is completely driven by packing entropy.

We note that the cubes display different orientations in the dilute fluid phase $f$, as a function of the Young contact angle $\theta$. They are indeed adsorbed in the minimum energy configuration at the $\theta$ of interest: the $\{111\}$ configuration at $\cos \theta=0$, the $\{100\}$ configuration at the other contact angles.

Finally, we summarize the results of Fig. 3.10 in the ground state $(T=0)$ phase diagram of Fig. 3.11. In this phase diagram we report the packing density $\eta$ on the horizontal axis, and the cosine of the contact angle $\theta$ on the vertical axis. We note that three regimes of $\theta$ 
exist, in which different phases stabilize by varying $\eta$. In the regime of large contact angles $(\cos \theta<0.35)$ the $f, x$, and $s_{\mathrm{f}}$ phases stabilize, in the regime of intermediate contact angles $(0.35 \leq \cos \theta \leq 0.4)$ the $f, x, c, s_{\mathrm{e}}$ and $s_{\mathrm{f}}$ phases stabilize, in the regime of small contact angles $(\cos \theta>0.4)$ the $f$, and $s_{\mathrm{f}}$ phases are stable. We note that in Fig. 3.11 we included the case of $\cos \theta=0.0$, for which a graph similar to those of Fig. 3.10 is reported in Fig. 3.12.

In the analysis performed so far we only have considered the effect of capillary interactions while we have completely neglected entropic contributions. Although this approximation is correct when studying the phase behaviour of $\mu$ m-sized particles as those of Refs. [31,32], it is less accurate when smaller particles are considered. We now extend the analysis to the case of nm-sized particles and introduce an approximate model to estimate the interplay between capillary interactions and particle entropy. We write the (Helmholtz) free energy $F^{\xi}$ of a system of $N$ colloidal particles adsorbed at a fluid-fluid interface of area $A$ and temperature $T$, in a particular phase $\xi$, as

$$
\frac{F^{\xi}}{\gamma \Sigma}=\frac{N \Delta E^{\xi}}{\gamma \Sigma}+N \frac{k_{\mathrm{B}} T}{\gamma \Sigma}\left(\ln \frac{\eta}{\left(1-\eta / \eta_{\mathrm{CP}}^{\xi}\right)}-1\right),
$$

where $\eta, \eta_{\mathrm{CP}}^{\xi}$ and $\Delta E$ have been defined above while $k_{\mathrm{B}}$ is the Boltzmann constant. We express $F^{\xi}$ in terms of $\gamma \Sigma$, with $\gamma$ the fluid-fluid surface tension, and $\Sigma$ the surface area of the particle. The second term on the right side of Eq. (3.2) is added in order to include entropic effects. It consists of the free energy of an ideal gas [124] plus the extra term $-\left(N k_{\mathrm{B}} T /(\gamma \Sigma)\right) \ln \left(1-\eta / \eta_{\mathrm{CP}}^{\xi}\right)$, which ensures the free energy to diverge at the close-packed density $\eta_{\mathrm{CP}}^{\xi}$ of phase $\xi$. Note that we include in our model entropic effects only in an approximated way, without any explicit reference to the particle shape and orientation entropy. Nevertheless, as we will show, this approximation reproduces remarkably well results, especially in the limit $\gamma \Sigma \gg k_{\mathrm{B}} T$. This is the limit of low temperatures or large particles, where the free energy (3.2) is dominated by the capillary contribution $\Delta E^{\xi}$ while the entropic term only contributes significantly close to the close-packed limit $\eta \rightarrow \eta_{\mathrm{CP}}^{\xi}$.

By considering $k_{\mathrm{B}} T /(\gamma \Sigma) \neq 0$ in Eq. (3.2), we map out the phase diagrams depicted in Fig. 3.13 for $\cos \theta \in\{0.2,0.35,0.4\}$. Here we represent the particle density $\eta$ on the horizontal axis, and the dimensionless temperature $k_{\mathrm{B}} T /(\gamma \Sigma)$ on the left vertical axis. The right vertical axis represents the corresponding value of the particle size $L$ at room temperature $(T=300 K)$ using a typical surface tension $\gamma=0.01 \mathrm{~N} \mathrm{~m}^{-1}$. We find that our results reproduce remarkably well the experimental observations in Refs. [31,32]. Indeed for $\mu \mathrm{m}$-sized $L$ we find that, by decreasing $\theta$, first hexagonal $x$ structures and then linear chains $c$ stabilize. In agreement with experiments we also predict regions in which the $x$ and the $c$ phases coexist, as well as the stabilization of "face up" square $s_{\mathrm{f}}$ patterns at very small $\theta \sim 0$. Furthermore we see that for $\cos \theta=0.4$ regions exist in which "edge up" square $s_{\mathrm{e}}$ aggregates stabilize in coexistence with either $f, c$, or $s_{\mathrm{f}}$ phases. For cubes with nm-sized $L$ we also find the presence of $f$-c-x, and $f-c-s_{\mathrm{e}}$ triple-points. Note that in the limit of high temperature $T$ our model for the free energy becomes less accurate and only the phase $f$ survives. For $k_{\mathrm{B}} T /(\gamma \Sigma)=0$, instead, the scenario depicted in Fig. 3.13 coincides with the ground states depicted in Figs. 3.10 and 3.11.

As a final remark we observe that we cannot build a proper phase diagram in the case of $\cos \theta=0.6$, where the capillary interactions become so weak that the phase behaviour is governed by packing entropy among cubes in the adsorbed "face up" $\{100\}$ configuration. A more accurate theoretical model of the free energy that takes into account the particle shape and excluded volume is then required to describe the entropy-driven phase transitions. 


\subsection{Conclusions and Discussion}

In this Chapter, we numerically studied the adsorption and self-assembly of cubic colloidal particles at a fluid-fluid interface, as a function of the Young contact angle $\theta$. For values of $\theta$ close to $\pi / 2$, we confirmed the scenario already found in a series of previous papers: a single cube prefers to adsorb with one vertex pointing upward with respect to the interface plane ("vertex up" configuration) and induces a hexapolar deformation field in the interface height profile which causes a lattice of $N \rightarrow \infty$ adsorbed cubes to assemble into "vertex up" hexagonal structures. At the same time, a cube with $\theta=\pi / 2$ adsorbed with one edge pointing upward ("edge up" configuration) or with one face pointing upward ("face up" configuration), does not induce any deformation in the interface height profile. By decreasing $\theta$, however, we found that the intensity of the hexapolar deformation field induced by a cube in the "vertex up" configuration decreases, while a cube in the "edge up" configuration induces a finite quadrupolar deformation field. The intensity of this quadrupole increases with decreasing $\theta$, and a regime exists in which it causes a lattice of $N \rightarrow \infty$ adsorbed cubes to assemble into stable "edge up" chains and square aggregates. Finally we observed that, by decreasing $\theta$ even further, the cubes give rise to "face up" square patterns, whose stabilization is driven by packing entropy as the capillary interactions become very weak.

Our results are in agreement with the findings of recent experiments performed on $\mu \mathrm{m}-$ sized polystyrene cubes. In particular they give strong theoretical support to the idea of a capillary-driven stabilization of chains of cubic colloids at fluid-fluid interfaces, in addition to the stabilization of other, already found, 2D structures. Our analysis also explains the linear aggregates of "edge up" cubes observed in the case of the cubic nanocrystals (of roughly 5-to$10 \mathrm{~nm}$ size) studied in Refs. [125-127]. Nevertheless for these experiments the true underlying mechanism (capillarity, van der Waals forces, steric interactions due to capping ligands,..) that drives the self-assembly is still under investigation.

We note that chains of cubic colloids have also been observed in systems of magnetic cubes with a fixed magnetic dipole moment and in suspensions of colloidal cubes in an electric field [128-133], inducing dipolar interactions between the cubes. As the physical mechanism behind the chain formation is very different from the capillary-induced chain formation of colloidal cubes at an interface, the structure of the chains can also be very different. Depending on the orientation of the fixed dipole moment of the magnetic cubes, the cubes within the chains can have different orientations, and in the case of dipolar cubes in an electric field, the cubes can even freely rotate along the field axis [133].

Finally several extensions of the work presented in this Chapter could be considered. First it could be interesting to investigate the effects of slightly tuning the shape of the cubic particles (i.e. considering cubes with edges truncated at different levels) on the self-assembly at the interface, as a function of the Young contact angle $\theta$. In addition, the simple model used here for the free energy could be improved to take into account, for instance the orientational entropy of the cubes in each phase. In Refs. $[33,34]$ it was shown that considering this contribution can lead to stable honeycomb and "vertex up" square patterns coexisting with hexagonal ones, for nm-sized cubes with $\theta=\pi / 2$. For smaller $\theta$ it could reveal the presence of coexistence regions between the chains with honeycomb and "vertex up" square patterns. We plan to address these issues in future work. 


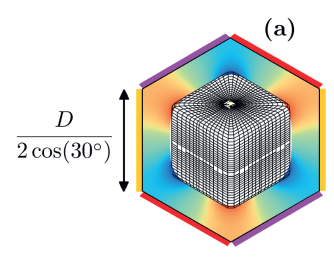

(d)

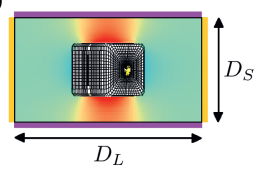

(b)

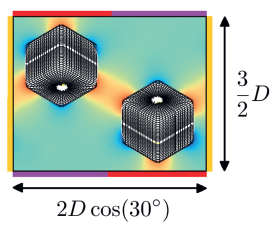

(e)

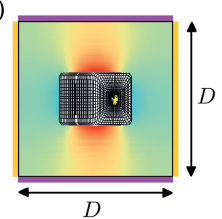

(c)

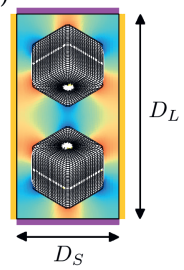

(f)

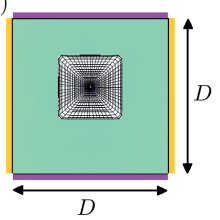

Figure 3.14: Lattice unit cells and the periodic boundary conditions (PBCs) used to define the several phases studied in Section 3.3.

\subsection{Acknowledgments}

The results presented in this Chapter are part of a collaboration with Dr. G. Soligno, who suggested to study the chainlike structures and provided the numerical code in the initial stage of the project.

\subsection{Appendix A: Periodic Boundary Conditions}

In this Appendix we discuss the lattice unit cells and the periodic boundary conditions (PBCs) used to define the several phases studied in Section 3.3. We refer to Fig. 3.14. Each unit cell is centered in $(x=0, y=0)$, and segments represented with the same colour have the same fluid-fluid interface height profile. For the hexagonal phase $x$ (see Fig. $3.14(a)$ ) we use a hexagonal unit cell with sides $D /\left(2 \cos \left(30^{\circ}\right)\right)$, where $D$ is the center-of-mass distance between two nearest-neighbor particles in the lattice. We apply the following PBCs to the cell sides: opposite sides of the hexagonal cell have the same fluid-fluid interface height profile. In the cell there is $N=1$ particle in the $\{111\}$ configuration, and with $\left(x_{1}, y_{1}, \alpha_{1}\right)$ given by $(0,0,0)$. For the honeycomb phase $h$ (see Fig. $3.14(b))$ we use a rectangular cell with sides $2 D \cos \left(30^{\circ}\right)$ and $3 D / 2$, respectively, where $D$ is the center-of-mass distance between two nearest-neighbor particles in the lattice. We apply the following PBCs to the cell sides: the half-side from $\left(-D \cos \left(30^{\circ}\right),-3 D / 4\right)$ to $(0,-3 D / 4)$ has the same fluid-fluid interface height profile of the halfside from $(0,3 D / 4)$ to $\left(D \cos \left(30^{\circ}\right), 3 D / 4\right)$, the half-side from $(0,-3 D / 4)$ to $\left(D \cos \left(30^{\circ}\right),-3 D / 4\right)$ has the same fluid-fluid interface height profile of the half-side from $\left(-D \cos \left(30^{\circ}\right), 3 D / 4\right)$ to $(0,3 D / 4)$, and the two remaining opposite sides of the cell have the same fluid-fluid interface height profile. In the cell there are $N=2$ particles in the $\{111\}$ configuration, and with $\left(x_{i}, y_{i}, \alpha_{i}\right)$, for $i=1,2$, given by $\left(-D \cos \left(30^{\circ}\right) / 2,3 D / 4,0\right)$ and $\left(D \cos \left(30^{\circ}\right) / 2,-3 D / 4, \pi\right)$. For the "vertex up" square phase $s_{\mathrm{v}}$ (see Fig. $3.14(c)$ ) we consider a rectangular unit cell with sides $D_{S}$ and $D_{L}$, respectively, where $D_{S}$ and $D_{L}$ are the center-of-mass distances between two nearest neighbor particles in the dipole-dipole bond direction of the lattice and in the tripoletripole bond direction of the lattice, respectively. We apply the following PBCs to the cell sides: 


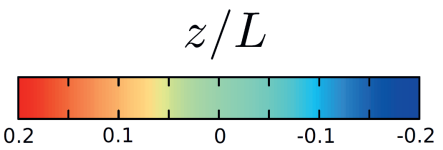

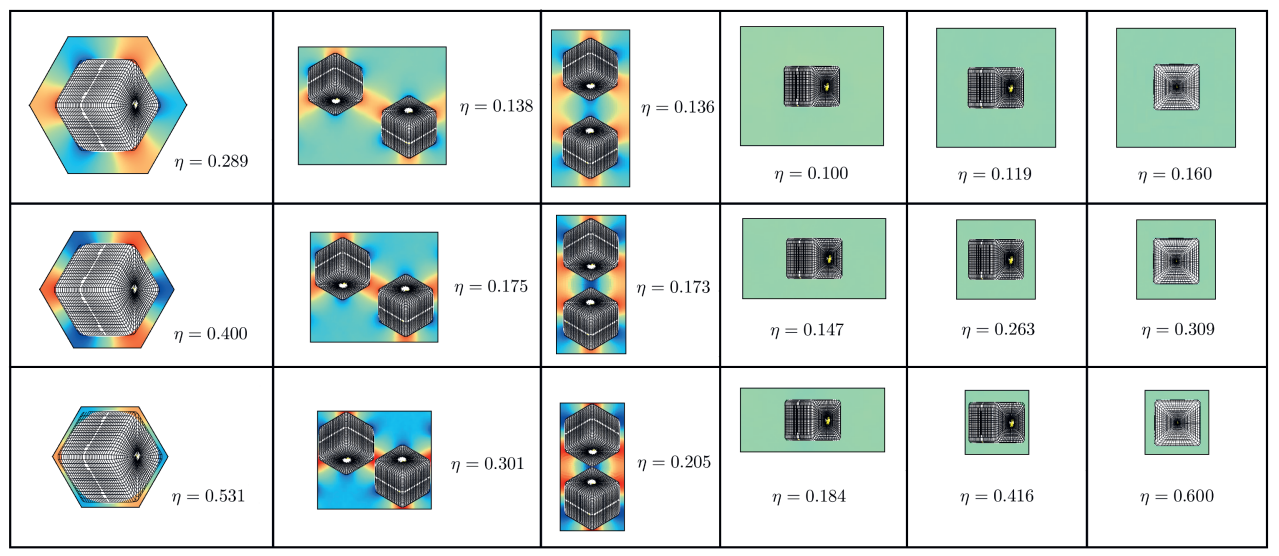

Figure 3.15: Contour plot of the fluid-fluid interface height profile, as obtained by our numerical method, in a unit cell of phase $x, h, s_{\mathrm{v}}, c, s_{\mathrm{e}}$ and $s_{\mathrm{f}}$ (from left to right, respectively) at a fixed Young contact angle $\cos \theta=0.0$. Note that PBCs are applied to the lattice unit cells, as described in Fig. 3.14. Each lattice unit cell is shown for a given packing fraction $\eta$. The plane $z=0$ corresponds to the fluid-fluid interface when no particle is adsorbed. With $L$ we denote the side of the cube.

opposite sides of the square cell have the same fluid-fluid interface height profile. In the cell there are $N=2$ particles in the $\{111\}$ configuration, and with $\left(x_{i}, y_{i}, \alpha_{i}\right)$ for $i=1$, 2 , given by $\left(0,-D_{L} / 2,0\right)$ and $\left(0, D_{L} / 2, \pi\right)$. For the chains phase $c$ (see Fig. $\left.3.14(d)\right)$ we use a rectangular unit cell with sides $D_{S}$ and $D_{L}$, respectively, where $D_{S}$ is the center-of-mass distance between two nearest-neighbor particles of the lattice. We instead choose $D_{L} \equiv D_{L}^{*}=3.0 L$, with $L$ the side length of the cubic particles. $D_{L}^{*}$ corresponds to the smallest distance for which two cubes in the $\{110\}$ configurations do not interact. In this way we describe the $c$ phase as a "gas" of chains that do not interact laterally. We apply the following PBCs to the cell sides: opposite sides of the cell have the same fluid-fluid interface height profile. In the cell there is $N=1$ particle in the $\{110\}$ configuration, and with $\left(x_{1}, y_{1}, \alpha_{1}\right)$ given by $(0,0,0)$. For the "edge up" square phase $s_{\mathrm{e}}$ (see Fig. $3.14(e)$ ) we use a square unit cell with side $D$, where $D$ is the center-of-mass distance between two nearest-neighbor particles of the lattice. We apply the following PBCs to the cell sides: opposite sides of the cell have the same fluid-fluid interface height profile. In the cell there is $N=1$ particle in the $\{110\}$ configuration, and with $\left(x_{1}, y_{1}, \alpha_{1}\right)$ given by $(0,0,0)$. For the "face up" square phase $s_{\mathrm{f}}$, (see Fig. $3.14(f)$ ) we use a square unit cell with sides $D$, where $D$ is the center-of-mass distance between two nearest-neighbor particles of the lattice. We apply the following PBCs to the cell sides: opposite sides of the cell have the same fluid-fluid interface height profile. In the cell there is $N=1$ particle in the $\{100\}$ configuration, and with $\left(x_{1}, y_{1}, \alpha_{1}\right)$ given by $(0,0,0)$.

By tuning $D$ for the phases $x, h, s_{\mathrm{e}}$, and $s_{\mathrm{f}}$, and $D_{S}, D_{L}$ for the phases $s_{\mathrm{v}}$ and $c$, we regulate the lattice spacing, and therefore the particle packing fraction $\eta$ in the lattice. $\eta$ is given by $\eta=2 /\left(\sqrt{3} D^{2}\right)$ for the phase $x$, by $\eta=4 /\left(3 \sqrt{3} D^{2}\right)$ for the phase $h$, by $\eta=1 /\left(D_{S} D_{L}\right)$ for the 

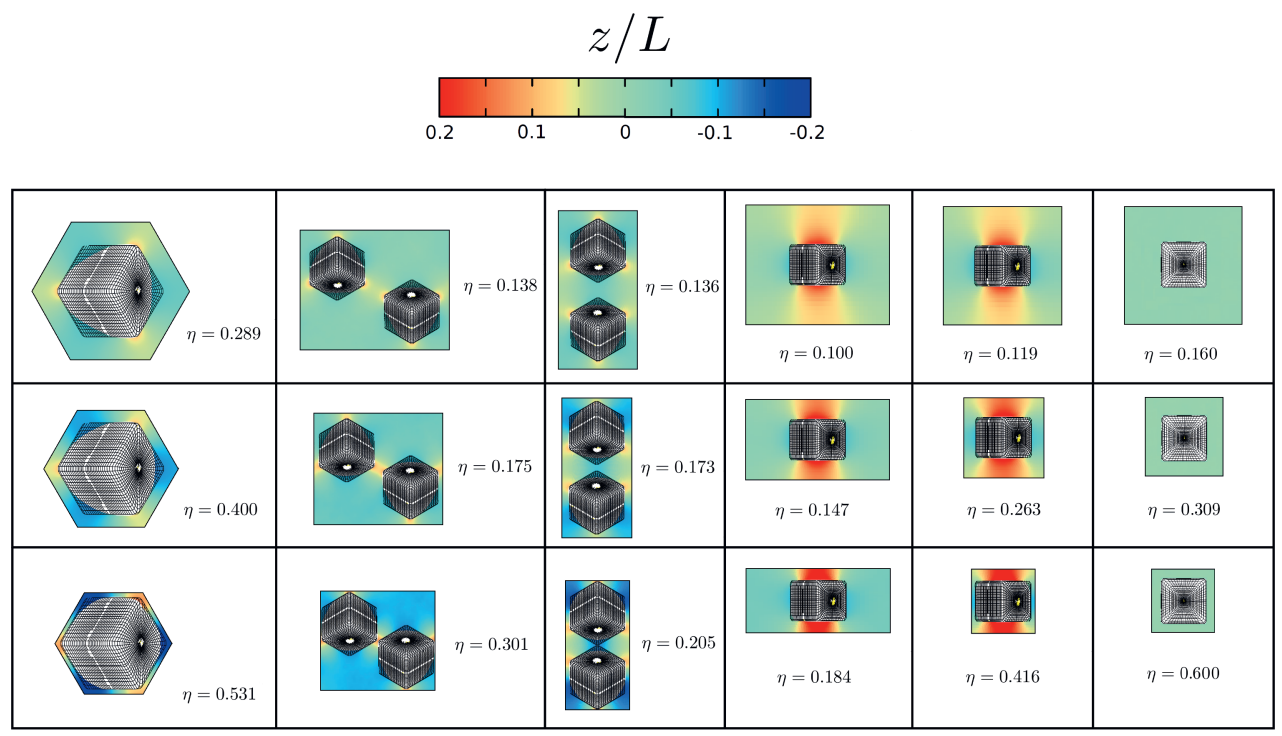

Figure 3.16: Contour plot of the fluid-fluid interface height profile, as obtained by our numerical method, in a unit cell of phase $x, h, s_{\mathrm{V}}, c, s_{\mathrm{e}}$ and $s_{\mathrm{f}}$ (from left to right, respectively) at a fixed Young contact angle $\cos \theta=0.4$. Note that PBCs are applied to the lattice unit cells, as described in Fig. 3.14. Each lattice unit cell is shown for a given packing fraction $\eta$. The plane $z=0$ corresponds to the fluid-fluid interface when no particle is adsorbed. With $L$ we denote the side of the cube.

phases $s_{\mathrm{v}}$ and $c$, and by $\eta=1 /\left(D^{2}\right)$ for the phases $s_{\mathrm{e}}$ and $s_{\mathrm{f}}$.

Examples of the application of the PBCs discussed in this Appendix are shown in Figs. (3.15) and (3.16), respectively. 


\section{4}

\section{A Landau-de Gennes theory for twist-bend and splay-bend nematic phases of colloidal suspensions of bent rods}

We develop a phenomenological Landau-de Gennes (LdG) theory for lyotropic colloidal suspensions of bent rods using a $Q$-tensor expansion of the chemical-potential dependent grand potential. In addition, we introduce a bend flexoelectric term, coupling the polarization and the divergence of the $Q$-tensor, to study the stability of uniaxial $(N)$, twist-bend $\left(N_{\mathrm{TB}}\right)$, and splay-bend $\left(N_{\mathrm{SB}}\right)$ nematic phases of colloidal bent rods. We first show that a mapping can be found between the LdG theory and the Oseen-Frank theory. By breaking the degeneracy between the splay and bend elastic constants, we find that the LdG theory predicts either an $N-N_{\mathrm{TB}}-N_{\mathrm{SB}}$ or an $N-N_{\mathrm{SB}}-N_{\mathrm{TB}}$ phase sequence upon increasing the particle concentration. Finally we employ our theory to study the first-order $N-N_{\text {TB }}$ phase transition, for which we find that the bend elastic constant $K_{33}$ as well as its renormalized version $K_{33}^{\text {eff }}$ remain positive at the transition, whereas $K_{33}^{\text {eff }}$ vanishes at the nematic spinodal. We connect these findings to recent simulation results. 


\subsection{Introduction}

Bent-core liquid crystals are mesophases formed by molecules with a "banana-like" shape [49]. In the simplest liquid crystal phase, i.e. the uniaxial nematic $(N)$ phase, the long axes of the bent-core molecules are preferentially aligned along a common direction, the so-called nematic director $\hat{\mathbf{n}}$, and the transverse orientations of the molecules are randomly oriented in the plane perpendicular to $\hat{\mathbf{n}}$. In addition to the $N$ phase with orientational order of the main molecular axis, the molecular shape can stabilize a nematic phase with polar order, in which the transverse orientations exhibit a net alignment in a direction perpendicular to $\hat{\mathbf{n}}$. In 1969 Meyer [82] argued that polar order of the transverse directions couples to the bend deformations of the nematic director $\hat{\mathbf{n}}$ through a mechanism called the bend flexoelectric effect. Polar order and the bend flexoelectric effect may occur in liquid crystals due to electrostatic polarization, but may also arise due to the molecular shape in systems of bent-core molecules in absence of electric fields. As a result, it is particularly easy to induce bend deformations in the nematic director field of bent-core liquid crystals. Many years later, Dozov [51] noted that the bend elastic constant $K_{33}$ can be very small for bent-core liquid crystals, yielding a low energy cost for bend deformations. In addition, Dozov speculated that $K_{33}$ could also become negative in certain bent-core liquid crystals. In this case, higher-order terms in the derivatives of the nematic director field (beyond linear elasticity) should be included in the free energy in order to stabilize the system. The competition between the putative negative $K_{33}$ term and the positive higher-order terms would favor spontaneous bend deformations. Interestingly, however, the theoretical work of Dozov did not consider any polar order. Since it is impossible to extend a pure bend deformation in three-dimensional space, Meyer as well as Dozov predicted that the uniaxial $N$ phase can either become unstable with respect to a spatially modulated twist-bend nematic $\left(N_{\mathrm{TB}}\right)$ phase, characterized by a heliconical variation with bend and twist deformations in the molecular orientation (see Fig. $4.1(\mathrm{a})$ ), or to a modulated splay-bend nematic $\left(N_{\mathrm{SB}}\right)$ phase, characterized by alternating domains of splay and bend [50,51] (see Fig. 4.1 (b)). Quantitatively, Dozov's theory, based on the Oseen-Frank elastic theory, predicts that the uniaxial $N$ phase becomes unstable to the formation of $N_{\mathrm{TB}}$ or $N_{\mathrm{SB}}$ phases at a critical point corresponding to $K_{33}=0$, where the system either stabilizes an $N_{\text {TB }}$ phase if $K_{11}>2 K_{22}$, or an $N_{\mathrm{SB}}$ phase if $K_{11}<2 K_{22}$, with $K_{11}$ and $K_{22}$ the splay and twist elastic constants, respectively [51].

Recently, Selinger and collaborators [52,53,134] suggested that the presence of polar order could provide the simplest explanation not only for the formation of spatially modulated phases, in agreement with Meyer, but also for the negative bend elastic constant $K_{33}$ proposed by Dozov. These authors introduced a Landau theory that combines the Oseen-Frank free energy for the nematic director $\hat{\mathbf{n}}$, the polar order $\mathbf{P}$ perpendicular to $\hat{\mathbf{n}}$, and a coupling between polar order and bend deformations. By minimizing the free energy with respect to polar order, they obtained Dozov's effective free energy in terms of only the nematic director field $\hat{\mathbf{n}}$ with renomalized elastic constants. In this picture, $K_{33}$ remains always positive while its renormalized version $K_{33}^{\text {eff }}$ decreases in magnitude and vanishes at a critical point where the uniaxial $N$ phase becomes unstable with respect to the $N_{\mathrm{TB}}$ or $N_{\mathrm{SB}}$ phase. Interestingly, they also found the same criterion for the relative stability of the spatially modulated phases calculated by Dozov [53], i.e. $K_{11}<2 K_{22}$ for a $N_{\mathrm{SB}}$ phase and $K_{11}>2 K_{22}$ for a $N_{\mathrm{TB}}$ phase. Finally, Selinger's theory has been extended [54,135-138] to a mesoscopic Landau-de Gennes $(\mathrm{LdG})$ theory where the director $\hat{\mathbf{n}}$ is replaced by a second rank, symmetric and traceless tensor $\mathbf{Q}(\mathbf{r})$ with components $Q_{\alpha \beta}(\mathbf{r})$ where $\alpha, \beta=1,2,3$ represent the Cartesian coordinates.

For completeness, we also mention that theories have been developed for bent-core liquid 
crystals that do not involve spontaneous polar order or a negative bend elastic constant [55-60]. Additionally, molecular field approaches [64,139-149] for bent-core liquid crystals exist, of which several $[139,145,147-149]$ support the idea of a softening of the bend elastic constant before the onset of polar order in bent-core liquid crystals, in agreement with Selinger et al. [52,53,134]

Much research in recent years has been focused on thermotropic bent-core mesogens that become liquid crystalline upon lowering the temperature. Very recently, various routes have been developed to synthesize lyotropic colloidal model systems of bent-core molecules, e.g. silica rods with a sharp kink [61-63] or smoothly curved SU-8 rods [66]. The liquid crystalline behavior of these colloidal systems is driven by concentration and has been studied by simulations and microscopic theories. Using Onsager theory [36], a first-order uniaxial $N$ to $N_{\mathrm{TB}}$ phase transition has been predicted recently in a system of hard curved particles at sufficiently high particle concentrations [64], which has been confirmed in computer simulations [64,65] on systems of hard bent spherocylinders. In addition, this simulation study showed that the $N$ $N_{\mathrm{TB}}$ phase transition is followed by a second-order $N_{\mathrm{TB}}-N_{\mathrm{SB}}$ phase transition in a polydisperse system of hard bent spherocylinders and in a system of hard curved particles.

In this Chapter, we extend the existing LdG theories of thermotropic bent-core liquid crystals to lyotropic liquid crystals in order to develop a framework to describe the recent findings of Refs. [64,65]. To this end, we introduce a chemical-potential dependent grand potential based on a $Q$-tensor expansion and a bend flexoelectric term coupling the polarization and the divergence of the $Q$-tensor [54,135-138]. We first show that a mapping can be found between the LdG theory and the Oseen-Frank theory of Selinger [53,134]. We then show, by breaking the degeneracy between the splay and bend elastic constants, that the LdG theory predicts a series of second-order phase transitions between periodically modulated nematic phases, reproducing what was found in Ref. [65]. Finally we employ our theory to study the first-order $N-N_{\text {TB }}$ phase transition observed in simulations. We find that while the LdG theory predicts that $K_{33}>0$ and $K_{33}^{\text {eff }}=0$ at a second-order $N-N_{\text {TB }}$ phase transition, it also predicts that $K_{33}$ as well as $K_{33}^{\text {eff }}$ remain positive at a first-order $N-N_{\text {TB }}$ transition, whereas $K_{33}^{\text {eff }}$ vanishes at the nematic spinodal.

As a final introductory remark it is worth mentioning that the splay-bend nematic phase considered in this Chapter differs from the so-called splay nematic $\left(N_{\mathrm{S}}\right)$ phase considered in Refs. $[138,150,151]$. This $N_{\mathrm{S}}$ phase is characterized by a modulation perpendicular to the average director while the $N_{\mathrm{SB}}$ phase is characterized by a spatial modulation parallel to the global nematic director. Moreover the onset of the $N_{\mathrm{S}}$ phase is driven by a softening of the renormalized splay elastic constant $K_{11}^{\mathrm{eff}}$ rather than of the renormalized bend elastic constant $K_{33}^{\mathrm{eff}}$.

The outline of this Chapter is as follows: Section 4.2 describes our LdG theory. In Section 4.3 we briefly review the isotropic-nematic phase transition of hard rods within this framework, that will be used as a reference system throughout the Chapter. In Section 4.4 we investigate possible phase sequences of the spatially modulated phases. The first-order $N-N_{\mathrm{TB}}$ transition is studied in Section 4.5 and the renormalized elastic constants are derived in Section 4.6. Finally we present our conclusions and a discussion in Section 4.7.

\subsection{Landau-de Gennes theory}

LdG theory is based on the hypothesis that equilibrium properties of a thermodynamic system can be found from a variational Helmholtz (or Gibbs) free energy $F$, constructed as an expansion 


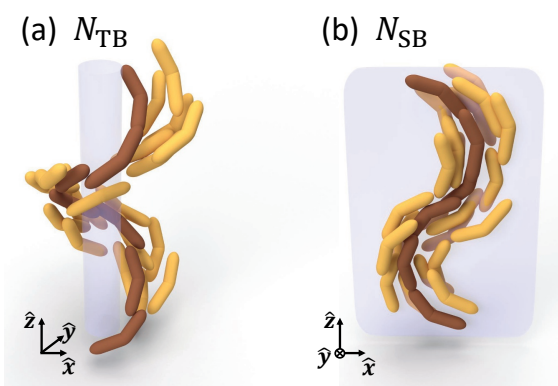

Figure 4.1: (a) Twist-bend nematic $\left(N_{\mathrm{TB}}\right)$ phase characterized by a heliconical variation of the particles orientation along the $z$-axis. (b) Splay-bend nematic $\left(N_{\mathrm{SB}}\right)$ phase characterized by alternating domains of splay and bend in the $x-z$ plane.

in powers of a suitable order parameter. A restriction on the expansion is that it must be stable against an unlimited growth of the order parameter. It is well-known [76] that the orientational order of three-dimensional nematic liquid crystals can be described by a secondrank, symmetric, traceless tensor field, $\mathbf{Q}(\mathbf{r})$ with cartesian components $Q_{\alpha \beta}(\mathbf{r})$ for $\alpha, \beta=1,2,3$, which vanishes in the isotropic $(I)$ phase and thus serves as an order parameter for the $N$ phase. The eigenvector of $\mathbf{Q}$ corresponding to the maximum modulus of a nondegenerate eigenvalue defines the nematic director $\hat{\mathbf{n}}$ of the system. The variational LdG free energy $F$ for ordinary, non-chiral nematics is constructed from frame-invariant contractions of $Q_{\alpha \beta}$ and spatial derivatives $\partial_{\lambda} Q_{\alpha \beta}$ such as $Q_{\alpha \beta} Q_{\beta \alpha}, Q_{\alpha \beta} Q_{\beta \lambda} Q_{\lambda \alpha}$, etc., with phenomenological coefficients that contain the dependence on the thermodynamic state (pressure, temperature). Usually for thermotropic liquid crystals, only the quadratic term of the Landau expansion changes sign as a function of temperature, which drives the phase transition $[79,152,153]$.

In contrast to "ordinary" nematics, a proper characterization of orientational order exhibited by bent-core liquid-crystal phases requires additional order parameters. In the case of theories based on the flexoelectric effect, not only the tensor field $\mathbf{Q}(\mathbf{r})$ is required, but also a vector field $\mathbf{P}(\mathbf{r})$ with cartesian components $P_{\alpha}(\mathbf{r})$ that describes the polar order in a direction perpendicular to $\hat{\mathbf{n}}$. In the $I$ phase $\mathbf{Q}=\mathbf{0}$ and $\mathbf{P}=\mathbf{0}$; in the uniaxial $N$ phase $\mathbf{Q} \neq \mathbf{0}$ and $\mathbf{P}=\mathbf{0}$; and in the spatially modulated nematic phases $\mathbf{Q} \neq \mathbf{0}$ and $\mathbf{P} \neq \mathbf{0}$. General $O(3)$-symmetric extensions of the free energy $F$ that contain additionally lowest order couplings with $\mathbf{P}$ and its derivatives $\partial_{\alpha} P_{\beta}$ have been developed in Refs. [54,135-138]. However, these expansions are only suitable for thermotropic systems that become liquid crystalline as a function of temperature. In contrast, lyotropic systems become ordered as a function of density and are not conveniently described by the Helmholtz free energy $F$. A naive remedy for this problem would be to replace the temperature in $F$ by the density $\rho$, but this cannot capture the density jumps that are found at first-order transitions, which for the $I-N$ phase transition can be as large as $25 \%$ [154]. The density discontinuity at the $I-N$ transition is instead exhibited by microscopic theories, such as Onsager theory.

Here we follow Ref. [80] and set up a Landau expansion for lyotropics for which we will use the grand potential $\Omega$ rather than the Helmholtz (or Gibbs) free energy $F$. By using $\Omega$, the expansion parameters will depend on the chemical potential $\mu$, and the density jumps will naturally be encoded through the relation $\partial(\Omega / V) /\left.\partial \mu\right|_{V, T}=-\rho$, with $V$ the volume of the system and $\rho$ the average density. Only the quadratic term $Q_{\alpha \beta} Q_{\beta \alpha}$ has a $\mu$-dependent 
prefactor that changes sign to drive the phase transition. This procedure is easier to use than, for example, the phase-field-crystal method of Ref. [155], which produces terms that also explicitly depend on density, for which also an Euler-Lagrange equation for $\rho$ needs to be solved, in addition to the one for $\mathbf{Q}$.

We consider a system of hard bent rods modeled as curved or kinked rods of contour length $L$ and diameter $D$, at chemical potential $\mu$ in a macroscopic volume $V$ at fixed temperature $T$. We write the LdG grand potential as

$$
\Delta \Omega(\mathbf{Q}, \mathbf{P})=\int_{V} d \mathbf{r}\left[\Delta \omega_{b}+\omega_{e}+\omega_{P}\right]
$$

where $\Delta \omega_{b} \equiv \Delta \omega_{b}(\mathbf{Q} ; \mu)$ is the excess bulk grand potential density with respect to the $I$ state, $\omega_{e} \equiv \omega_{e}(\mathbf{Q}, \nabla \mathbf{Q})$ describes elastic deformations and surface tension effects, and $\omega_{P} \equiv$ $\omega_{P}(\mathbf{Q}, \mathbf{P}, \nabla \mathbf{Q}, \nabla \mathbf{P})$ contains additionally lowest order couplings between $\mathbf{Q}$ and the polarization field $\mathbf{P}$ and its derivatives $\partial_{\alpha} P_{\beta}$.

We expand the bulk contribution in units of $\beta^{-1}=k_{B} T$ with $k_{B}$ the Boltzmann constant, until fourth order in $\mathbf{Q}$, which gives us

$$
\beta B_{2} \Delta \omega_{b}(\mathbf{Q} ; \mu)=\frac{2}{3} a \beta\left(\mu^{*}-\mu\right) Q_{\alpha \beta} Q_{\beta \alpha}-\frac{4}{3} b Q_{\alpha \beta} Q_{\beta \lambda} Q_{\lambda \alpha}+\frac{4}{9} d Q_{\alpha \beta} Q_{\beta \alpha} Q_{\lambda \rho} Q_{\rho \lambda},
$$

where we use Einstein's summation convention for repeated indices throughout this Chapter. The second virial coefficient in the isotropic fluid phase is given by $B_{2}=\pi L^{2} D / 4$ in the limit $L \gg D$ and is included in our definition to render the Landau coefficients $a, b$ and $d$ conveniently dimensionless. For simplicity we assume them to be independent of $\mu$. We also introduce $\mu^{*}$, the chemical potential at which the quadratic term changes sign, i.e. it defines the spinodal of the $I-N$ transition. A stable $I$ phase at $\mu<\mu^{*}$ requires $a>0$, the stability of the expansion (4.2) with respect to an unlimited growth of $\mathbf{Q}$ requires that $d>0$ while $b>0$ allows us to describe a first-order $I-N$ transition to a state with $\mathbf{Q} \neq \mathbf{0}$. Throughout we will satisfy these stability criteria.

For the terms in gradients of $\mathbf{Q}$ we only retain terms up to the square gradients in $\mathbf{Q}$, and we consider only one of the possible invariants that involve a coupling between the order parameter $\mathbf{Q}$ and quadratic gradient in $\mathbf{Q}$ to break the degeneracy between the splay and bend elastic constants $K_{11}$ and $K_{33}$ [81]. We thus write

$$
\beta B_{2} \omega_{e}(\mathbf{Q}, \nabla \mathbf{Q})=\frac{2}{9} l_{1}\left(\partial_{\alpha} Q_{\beta \lambda}\right)\left(\partial_{\alpha} Q_{\beta \lambda}\right)+\frac{2}{9} l_{2}\left(\partial_{\alpha} Q_{\alpha \lambda}\right)\left(\partial_{\beta} Q_{\beta \lambda}\right)-\frac{2}{9} l_{3} Q_{\alpha \beta}\left(\partial_{\gamma} Q_{\alpha \gamma}\right)\left(\partial_{\xi} Q_{\beta \xi}\right),
$$

where we omitted another second-order term in $\nabla \mathbf{Q}$, that scales with $\left(\partial_{\alpha} Q_{\beta \lambda}\right)\left(\partial_{\lambda} Q_{\beta \alpha}\right)$ because it can be written as a linear combination of a surface term and the elastic terms already included in the expansion (4.3). We express the expansion parameters $l_{1}, l_{2}$ and $l_{3}$ in units of $L^{2}$ throughout the paper. We note again that we have chosen only one of the possible couplings between $\mathbf{Q}$ and $\nabla \mathbf{Q}$ to break the degeneracy between $K_{11}$ and $K_{33}$ [81]. This choice is arbitrary, also other terms could have been considered or even more terms could have been included. Since all couplings add a contribution proportional to $S^{3}$ to the elastic constants, the predictions of the theory are not affected by this choice.

Expressing $\mathbf{Q}(\mathbf{r})$ in terms of a scalar order parameter $S(\mathbf{r})$ and a nematic director field $\mathbf{n}(\mathbf{r})$,

$$
Q_{\alpha \beta}(\mathbf{r})=\frac{3}{2} S(\mathbf{r})\left(n_{\alpha}(\mathbf{r}) n_{\beta}(\mathbf{r})-\frac{1}{3} \delta_{\alpha \beta}\right),
$$


we can relate the parameters $l_{1}, l_{2}$ and $l_{3}$ to the Oseen-Frank elastic constants through $\beta D K_{11}=$ $4 S^{2}\left(2 l_{1}+l_{2}-S l_{3}\right) /\left(\pi L^{2}\right), \beta D K_{22}=8 S^{2} l_{1} /\left(\pi L^{2}\right)$, and $\beta D K_{33}=4 S^{2}\left(2 l_{1}+l_{2}+(S / 2) l_{3}\right) /\left(\pi L^{2}\right)$. These relations can be found by comparing the elastic expansion (4.3) using the expression (4.4) with the Oseen-Frank elastic energy [74,75]

$$
F=\frac{1}{2} \int d \mathbf{r}\left[K_{11}(\nabla \cdot \hat{\mathbf{n}})^{2}+K_{22}(\hat{\mathbf{n}} \cdot \nabla \times \hat{\mathbf{n}})^{2}+K_{33}|\hat{\mathbf{n}} \times(\nabla \times \hat{\mathbf{n}})|^{2}\right],
$$

where $K_{11}, K_{22}$ and $K_{33}$ are the splay, twist and bend elastic constants, respectively. We assume $l_{1}, l_{2}$ and $l_{3}$ to be independent of $\mu$.

Finally we expand $\omega_{P}$ up to sixth order in $\mathbf{P}$, and write

$$
\begin{aligned}
\beta B_{2} \omega_{P}(\mathbf{Q}, \mathbf{P}, \nabla \mathbf{Q}, \nabla \mathbf{P})= & e_{2} P_{\alpha}\left(\delta_{\alpha \beta}+\frac{2}{S_{0}} Q_{\alpha \beta}\right) P_{\beta}+e_{4} P_{\alpha} P_{\alpha} P_{\beta} P_{\beta}-\lambda P_{\alpha}\left(\partial_{\beta} Q_{\alpha \beta}\right)+\kappa\left(\partial_{\alpha} P_{\beta}\right)\left(\partial_{\alpha} P_{\beta}\right) \\
& +e_{6} P_{\alpha} P_{\alpha} P_{\beta} P_{\beta} P_{\gamma} P_{\gamma},
\end{aligned}
$$

with coefficients $e_{2}, e_{4}, e_{6}, \kappa, \lambda$ and $S_{0}$. Throughout the Chapter we will express $\lambda$ and $\kappa$ in terms of $L$ and $L^{2}$, respectively. Stability in the dilute limit requires $e_{2}>0$ while stability with respect to an unlimited growth of $\mathbf{P}$ requires that $e_{6}>0$. The coefficients $2 / S_{0}, \lambda$, and $\kappa$ represent the strength of the coupling between $\mathbf{Q}$ and $\mathbf{P}$ fields, the flexoelectric coupling between $\mathbf{P}$ and gradients in $\mathbf{Q}$, and a polar elastic constant, respectively. In order to describe a favored polarization perpendicular to the nematic director, leading to bend flexoelectricity, we set $S_{0}>0$ [54]. Finally, we allow $e_{4}$ to be positive as well as negative.

Our LdG expansion is very similar to those of Refs. [54,137]. Nevertheless, in contrast with their work, and following the suggestion of Ref. [134], we consider terms up to sixth order in $\mathbf{P}$ and simultaneously allow the coefficient $e_{4}$ of the fourth-order term in $\mathbf{P}$ to be either positive or negative. This choice allows us to describe first-order as well as second-order transitions to the spatially modulated phases [156]. In particular if $e_{4}<0$ we expect first-order phase transitions, while if $e_{4} \geq 0$ we expect second-order phase transitions. Our expansion includes the additional elastic term $Q_{\alpha \beta}\left(\partial_{\gamma} Q_{\alpha \gamma}\right)\left(\partial_{\xi} Q_{\beta \xi}\right)$ to break the degeneracy between the splay and bend elastic constants $K_{11}$ and $K_{33}$ in agreement with Ref. [150]. However, we note that Ref. [150] lacks the term $\left(\partial_{\alpha} Q_{\alpha \lambda}\right)\left(\partial_{\beta} Q_{\beta \lambda}\right)$ and only considers terms up to second-order in $\mathbf{P}$. We also remark that our additional elastic term allows us not only to break the degeneracy between the splay and bend elastic constants but also enables us to change the ratio between the splay and twist elastic constants $K_{11} / K_{22}$ by varying the particle concentration. As will become clear in Section 4.4, this latter condition is important for investigating the possibility of concentration-driven phase transitions between the periodically modulated phases, as was recently found in simulations of hard particles [65].

\section{$4.3 \quad$ I-N transition}

Here we briefly review the LdG theory to describe the $I-N$ transition of uniaxial hard rods as derived in Ref. [80]. As stated in the previous Section, the uniaxial $N$ phase is characterised by $\mathbf{Q} \neq \mathbf{0}$ and $\mathbf{P}=\mathbf{0}$, and hence the $\omega_{P}$ term in the grand potential (4.1) vanishes. We describe the bulk uniaxial $N$ phase by taking $\hat{\mathbf{n}}$ parallel to the $z$-axis. In this case the elastic expansion $\omega_{e}=0$ and $\Delta \Omega / V$ reduces to $\Delta \omega_{b}$. Inserting the tensor order parameter (4.4) with $\hat{\mathbf{n}}=(0,0,1)$ in $(4.2)$, we obtain $\beta B_{2} \Delta \omega_{b}=a \beta\left(\mu^{*}-\mu\right) S^{2}-b S^{3}+d S^{4}$. The Euler-Lagrange 
equation $\partial \Delta \omega_{b} / \partial S=0$ can be solved analytically, in order to find the stable, metastable and unstable phases. We find the solutions

$$
\begin{aligned}
& S_{I}(\mu)=0 ; \\
& S_{N}^{ \pm}(\mu)=\frac{3 b}{8 d}\left(1 \pm \sqrt{1-\frac{32 a d \beta\left(\mu^{*}-\mu\right)}{9 b^{2}}}\right),
\end{aligned}
$$

whose stability can be investigated by analyzing the sign of $\partial^{2} \Delta \omega_{b} / \partial S^{2}$. We first note that from the conditions $\partial^{2} \Delta \omega_{b} /\left.\partial S^{2}\right|_{S=S_{I}}=0, \partial^{2} \Delta \omega_{b} /\left.\partial S^{2}\right|_{S=S_{N}^{+}}=0$ and $\Delta \omega_{b}\left(S_{I}\right)=\Delta \omega_{b}\left(S_{N}^{+}\right)$, we can find the chemical potential $\mu^{*}$ corresponding to the spinodal of the $I$ phase with respect to the $N$ phase, the chemical potential $\beta \mu^{+}=\beta \mu^{*}-9 b /(32 a d)$ corresponding to the spinodal of the $N$ phase with respect to the $I$ phase, and the chemical potential $\beta \mu_{I N}=\beta \mu^{*}-b^{2} /(4 a d)$ corresponding to the $I-N$ transition, respectively. We then find that (i) for $\mu<\mu^{+}$the $I$ phase $\left(S_{I}\right)$ is the stable configuration, (ii) for $\mu^{+}<\mu<\mu_{I N}$ the $I$ phase is stable, $S_{N}^{-}$unstable, and $S_{N}^{+}$metastable, (iii) for $\mu_{I N}<\mu<\mu^{*}$ the $S_{N}^{+}$solution is stable, the $I$ phase is metastable, and $S_{N}^{-}$unstable, (iv) for $\mu>\mu^{*}$ the $S_{N}^{+}$solution is stable, $S_{N}^{-}$is metastable, and the $I$ phase is unstable. The solution $S_{N}^{+}$represents the $N$ phase and, for the sake of simplicity we will use $S_{N}(\mu) \equiv S_{N}^{+}(\mu)$ throughout this Chapter.

In order to describe the $I-N$ transition of hard rods, we first convert the chemical potential $\mu$ to the particle concentration $c=B_{2} \rho$, and then fit the phenomenological coefficients $a, b$, and $d$ to results from Onsager theory [80]. Concerning the first, we introduce the grand potential density of the $I$ state $\omega_{I}$ and define $\omega \equiv \omega_{I}+\Delta \omega_{b}$. From the condition $\partial\left(B_{2} \omega\right) / \partial \mu=-c$, we then find

$$
c(\mu)=c_{I}(\mu)+a S^{2}(\mu),
$$

where the particle concentration of the $I$ phase $c_{I}(\mu)=-\partial\left(B_{2} \omega_{I}\right) / \partial \mu$ can be calculated within Onsager theory, by using an isotropic distribution function, such that $\beta \mu\left(c_{I}\right)=\log \left(c_{I} / 4 \pi\right)+2 c_{I}$ [157]. By inverting this relation, we obtain $c_{I}(\mu)$. For the fit of the phenomenological coefficients $a, b$, and $d$, we exploit the thermodynamic quantities of the system at the $I-N$ phase coexistence. Using Onsager theory for a system of hard rods in the limit $L / D \rightarrow \infty[157,158]$, we find $c_{I}\left(\mu_{I N}\right)=3.290, c\left(\mu_{I N}\right)=4.191, \beta \mu^{*}=6.855, \beta \mu_{I N}=5.241$, and $S_{I N}=0.7992$. Inserting these values into the following expressions

$$
\begin{aligned}
& c\left(\mu_{I N}\right)=c_{I}\left(\mu_{I N}\right)+a S_{I N}^{2}, \\
& \beta \mu_{I N}=\beta \mu^{*}-b^{2} /(4 a d), \\
& S_{I N}=b /(2 d),
\end{aligned}
$$

we obtain $a=1.436, b=5.851$ and $d=3.693$. With this set of coefficients, a plot of $S_{I}$ and $S_{N}$ as a function of the concentration $c$ as defined by Eq. (4.8), allows one to observe that the concentration jump associated with the $I-N$ transition is correctly captured, as shown in Ref. [80]. This density jump is also shown in Fig. 1.6(b) of the main Introduction to this thesis. Unless stated otherwise, we will use these values of $a, b, d$, and $\mu^{*}$ in the following. We will also follow Ref. [80] in fitting the square-gradient coefficients $l_{1}$ and $l_{2}$ by using the surface tension of a planar $I-N$ interface of a system of hard rods with a parallel and perpendicular anchoring, yielding $l_{1}=0.165 L^{2}$ and $l_{2}=0.854 L^{2}$. We thus take a system of hard rods with $L / D \rightarrow \infty$ as a reference system for our study. Alternative reference systems are straightforward to implement provided a sufficient number of quantities is known at the bulk $I-N$ transition. 


\subsection{Spatially modulated phases}

In this Section we study the phase behaviour of lyotropic suspensions of bent rods by employing the LdG theory introduced in Section 4.2 with $e_{6}=0$ and $e_{4}>0$ in Eq. (4.6), i.e. using the formalism for second-order phase transitions. We show that if $l_{3} \neq 0$ in Eq. (4.3), the LdG theory predicts that an $N-N_{\mathrm{TB}}-N_{\mathrm{SB}}$ and an $N-N_{\mathrm{SB}}-N_{\mathrm{TB}}$ phase sequence can be stabilized in this system upon increasing the nematic order, in addition to the $N-N_{\mathrm{TB}}$ and $N-N_{\mathrm{SB}}$ transitions already predicted by the same theory in the case of $l_{3}=0$.

To this end, we describe the $N_{\text {ТВ }}$ phase by a nematic director $\hat{\mathbf{n}}_{\mathrm{TB}}(z)$ precessing around the $z$-axis with a conical angle $\theta$, a pitch $p=2 \pi / q$, and a polarization vector $\mathbf{P}_{\mathrm{TB}}(z)$ perpendicular to $\hat{\mathbf{n}}_{\mathrm{TB}}(z)$, given by $[51,53]$

$$
\begin{aligned}
& \hat{\mathbf{n}}_{\mathrm{TB}}(z)=(\sin \theta \cos (q z), \sin \theta \sin (q z), \cos \theta), \\
& \mathbf{P}_{\mathrm{TB}}(z)=P(\sin (q z),-\cos (q z), 0) .
\end{aligned}
$$

We describe the $N_{\mathrm{SB}}$ phase by a nematic director $\hat{\mathbf{n}}_{\mathrm{SB}}(z)$ and polarization vector $\mathbf{P}_{\mathrm{SB}}(z)$ given by $[51,53]$

$$
\begin{aligned}
& \hat{\mathbf{n}}_{\mathrm{SB}}(z)=(\sin \phi(z), 0, \cos \phi(z)) \\
& \mathbf{P}_{\mathrm{SB}}(z)=P \psi(z)\left(-\cos ^{2} \phi(z), 0, \frac{1}{2} \sin 2 \phi(z)\right),
\end{aligned}
$$

where $\psi(z)=\cos (q z)$ and $\phi(z)=\theta \sin q z$. Observe that $\hat{\mathbf{n}}_{\mathrm{SB}}$ describes alternating domains of splay and bend.

In order to study the stability of these phases, we first find the equilibrium values of $q$ and $\theta$, which can then be inserted into Eq. (4.1). Subsequently, we minimize the obtained $\Omega$ with respect to $S$ and $P$ at fixed $\mu$, i.e. we solve the Euler-Lagrange equations $\partial \Delta \Omega / \partial S=0$ and $\partial \Delta \Omega / \partial P=0$. However, since we are considering second-order transitions from the uniaxial $N$ phase, the dependence of $S$ on $\mu$ is known analytically, given by the $S_{N}(\mu)$ solution of Eq. (4.7). As a consequence, we can perform the stability analysis by minimizing $\Omega$ with respect to $P$ at fixed $S$, i.e. by solving the Euler-Lagrange equation $\partial \Delta \Omega / \partial P=0$. The dependence of $P$ and $S$ on the particle concentration can finally be obtained from the dependence on $\mu$ using the procedure described in Section 4.3.

\subsubsection{Comparison with the Oseen-Frank theory}

As already mentioned in the Introduction, the Oseen-Frank theory of Dozov [51] and Selinger $[53,134]$ predicts a phase transition from a uniaxial $N$ phase to either an $N_{\mathrm{TB}}$ or an $N_{\mathrm{SB}}$ phase. In this subsection, we show that a complete mapping exists between the LdG theory with $l_{3}=0$, i.e. with a degenerate bend and splay elastic constant, and the Oseen-Frank theory of Selinger [53, 134].

To compute the grand potential density of the $N_{\mathrm{TB}}$ phase, we insert $\hat{\mathbf{n}}_{\mathrm{TB}}(z)$ in the tensor order parameter Eq. (4.4) and the resulting $\mathbf{Q}(z)$ together with $\mathbf{P}_{\mathrm{TB}}(z)$ in the grand potential Eq. (4.1). We minimize the obtained grand potential with respect to the wave number $q$ and the tilt angle $\theta$, respectively, and find

$$
q_{\mathrm{TB}}=\frac{3 \lambda \sin \left(2 \theta_{\mathrm{TB}}\right) S P}{8 \kappa P^{2}+4 S^{2}\left(2 l_{1}+l_{2}\right) \sin ^{2} \theta_{\mathrm{TB}}-4 S^{2} l_{2} \sin ^{4} \theta_{\mathrm{TB}}},
$$



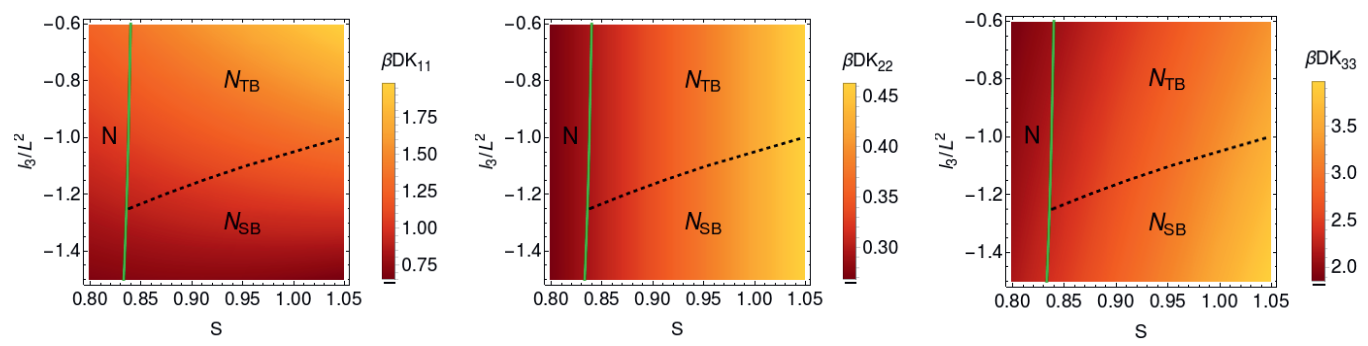

Figure 4.2: Phase diagram as a function of the scalar nematic order parameter $S$ and the coefficient $l_{3}$ for the coefficients $e_{2}=1, S_{0}=0.85, \kappa=0.1 L^{2}$ and $\lambda=0.1 L$, replicated three times with the background color denoting the value of the splay $\left(K_{11}\right)$, twist $\left(K_{22}\right)$, and bend $\left(K_{33}\right)$ elastic constants as indicated by the color bar. The $I-N$ phase transition occurs at $S_{I N}=0.7922$. The green line corresponds to the set of points where the renormalized bend elastic constant $K_{33}^{\text {eff }}$ vanishes and hence the uniaxial $N$ phase becomes unstable with respect to the spatially modulated nematic phases. For $l_{3}>-1.0 L^{2}$ and $l_{3}<-1.25 L^{2}$ only a second-order $N-N_{\mathrm{TB}}$ and $N-N_{\mathrm{SB}}$ phase transition occur as a function of $S$. For $-1.25 L^{2}<l_{3}<-1.0 L^{2}$, the $N-N_{\mathrm{TB}}$ phase transition is followed by a second-order $N_{\mathrm{TB}}-N_{\mathrm{SB}}$ phase transition.

and

$$
\sin ^{2} \theta_{\mathrm{TB}}=\frac{\kappa P^{2}}{S^{2} l_{1}}+\frac{\sqrt{\kappa P^{2}\left(\kappa P^{2}+S^{2} l_{1}\right)}}{S^{2} l_{1}} .
$$

Inserting Eqs. (4.12) and (4.13) back into Eq. (4.1) and approximating for small $P$, we find the grand potential density of the $N_{\mathrm{TB}}$ phase

$$
\begin{aligned}
\frac{\Delta \Omega_{\mathrm{TB}}}{V} & \equiv \frac{\Delta \Omega}{V}-\frac{\Delta \Omega_{N}}{V} \\
& =\left[\frac{e_{2}\left(S_{0}-S\right)}{S_{0}}-\frac{9 \lambda^{2}}{8\left(2 l_{1}+l_{2}\right)}\right] P^{2}+\frac{9 \lambda^{2} \sqrt{\kappa S^{2} l_{1}}}{2 S^{2}\left(2 l_{1}+l_{2}\right)^{2}}|P|^{3}+e_{4} P^{4}+O\left(P^{5}\right),
\end{aligned}
$$

where $\Delta \Omega_{N} / V=a S^{2}\left(\mu^{*}-\mu\right)-b S^{3}+d S^{4}$ is the grand potential density of the $N$ phase.

Analogously, in order to compute the grand potential density of the $N_{\mathrm{SB}}$ phase, we insert the nematic director $\hat{\mathbf{n}}_{\mathrm{SB}}(z)$ into the tensor order parameter Eq. (4.4) and the resulting $\mathbf{Q}(z)$ together with $\mathbf{P}_{\mathrm{SB}}(z)$ into the grand potential Eq. (4.1). In contrast with the case of the $N_{\mathrm{TB}}$ phase, the resulting grand potential density varies periodically as a function of $z$ and hence we average it over a full period $2 \pi / q$ to find

$$
\frac{\Delta \Omega}{V}=\frac{q}{2 \pi} \int_{0}^{\frac{2 \pi}{q}} d z \Delta \omega(z) .
$$

We then minimize the obtained grand potential with respect to the wave number $q$ and the tilt angle $\theta$, respectively, and find

$$
q_{\mathrm{SB}}=\frac{3 \lambda \theta_{\mathrm{SB}}\left(\theta_{\mathrm{SB}}^{2}-8\right) P S}{8\left(4+3 \theta_{\mathrm{SB}}^{2}\right) \kappa P^{2}+16 S^{2}\left(2 l_{1}+l_{2}\right) \theta_{\mathrm{SB}}^{2}},
$$


and

$$
\theta_{\mathrm{SB}}^{2}=\frac{16 \kappa P^{2}}{3 \kappa P^{2}+\sqrt{\kappa P^{2}\left(57 \kappa P^{2}+32 S^{2}\left(2 l_{1}+l_{2}\right)\right)}} .
$$

Inserting Eqs. (4.16) and (4.17) back into Eq. (4.1) and approximating for small $P$, we find for the grand potential density of the $N_{\mathrm{SB}}$ phase

$$
\begin{aligned}
\frac{\Delta \Omega_{\mathrm{SB}}}{V} & \equiv \frac{\Delta \Omega}{V}-\frac{\Delta \Omega_{N}}{V} \\
& =\left[\frac{e_{2}\left(S_{0}-S\right)}{2 S_{0}}-\frac{9 \lambda^{2}}{16\left(2 l_{1}+l_{2}\right)}\right] P^{2}+\frac{9 \lambda^{2} \sqrt{\kappa S^{2}\left(2 l_{1}+l_{2}\right)}}{8 \sqrt{2} S^{2}\left(2 l_{1}+l_{2}\right)^{2}}|P|^{3}+\frac{3 e_{4}}{8} P^{4}+O\left(P^{5}\right) .
\end{aligned}
$$

We observe that for small $P$ both $\Delta \Omega_{\mathrm{TB}}$ and $\Delta \Omega_{\mathrm{SB}}$ vanish at the critical scalar nematic order parameter

$$
S_{c}=S_{0}\left(1-\frac{9 \lambda^{2}}{8 e_{2}\left(2 l_{1}+l_{2}\right)}\right) .
$$

Close to this point we can assume

$$
P_{\mathrm{TB}} \ll 9 \lambda^{2} \sqrt{\kappa S^{2} l_{1}} /\left(2 e_{4} S^{2}\left(2 l_{1}+l_{2}\right)^{2}\right)
$$

and

$$
P_{\mathrm{SB}} \ll 3 \lambda^{2} \sqrt{\kappa S^{2}\left(2 l_{1}+l_{2}\right)} /\left(\sqrt{2} e_{4} S^{2}\left(2 l_{1}+l_{2}\right)^{2}\right),
$$

such that the cubic terms dominate over the quartic terms in Eqs. (4.14) and (4.18). Solving the Euler-Lagrange equations $\partial\left(\Delta \Omega_{\mathrm{TB}} / V\right) / \partial P=0$ and $\partial\left(\Delta \Omega_{\mathrm{SB}} / V\right) / \partial P=0$, we find

$$
P_{\mathrm{TB}}=\frac{4 e_{2} S^{2}\left(2 l_{1}+l_{2}\right)^{2}\left(S-S_{c}\right)}{27 S_{0} \lambda^{2} \sqrt{\kappa S^{2} l_{1}}},
$$

and

$$
P_{\mathrm{SB}}=\frac{8 \sqrt{2} e_{2} S^{2}\left(2 l_{1}+l_{2}\right)^{2}\left(S-S_{c}\right)}{27 S_{0} \lambda^{2} \sqrt{\kappa S^{2}\left(2 l_{1}+l_{2}\right)}},
$$

respectively. Inserting Eqs. (4.22) and (4.23) back in Eqs. (4.14) and (4.18), yields

$$
\frac{\Delta \Omega_{\mathrm{TB}}}{V}=-\frac{16 e_{2}^{3} S^{2}\left(2 l_{1}+l_{2}\right)^{4}\left(S-S_{c}\right)^{3}}{729 S_{0}^{3} \kappa \lambda^{4} l_{1}},
$$

and

$$
\frac{\Delta \Omega_{\mathrm{SB}}}{V}=-\frac{64 e_{2}^{3} S^{2}\left(2 l_{1}+l_{2}\right)^{3}\left(S-S_{c}\right)^{3}}{729 S_{0}^{3} \kappa \lambda^{4}} .
$$

The ratio between the grand potential densities (4.24) and (4.25) is then given by

$$
\frac{\Delta \Omega_{\mathrm{TB}}}{\Delta \Omega_{\mathrm{SB}}}=\frac{2 l_{1}+l_{2}}{4 l_{1}}=\frac{K_{11}}{2 K_{22}},
$$

where we have used that $K_{11}=S^{2}\left(2 l_{1}+l_{2}\right)$ and $K_{22}=S^{2}\left(2 l_{1}\right)$. From Eq. (4.26) and the overal minus signs in Eqs. (4.24) and (4.25), it is clear that at $S=S_{c}$ a second-order $N$ - $N_{\mathrm{TB}}$ occurs if $K_{11}>2 K_{22}$, while a second-order $N-N_{\mathrm{SB}}$ occurs if $K_{11}<2 K_{22}$, in perfect agreement with the findings of Selinger as well as of Dozov [51,53]. As will be shown in Section 4.6, at $S_{c}$ the renormalized elastic constant $K_{33}^{\text {eff }}$ vanishes while $K_{33}$ remains positive. A complete mapping of our LdG theory with the Oseen-Frank theory of Selinger follows. In particular it can be observed that Eqs. (4.22), (4.23), (4.24) and (4.25) strongly resemble the expressions for the free-energy differences given in Refs. [53, 134]. 

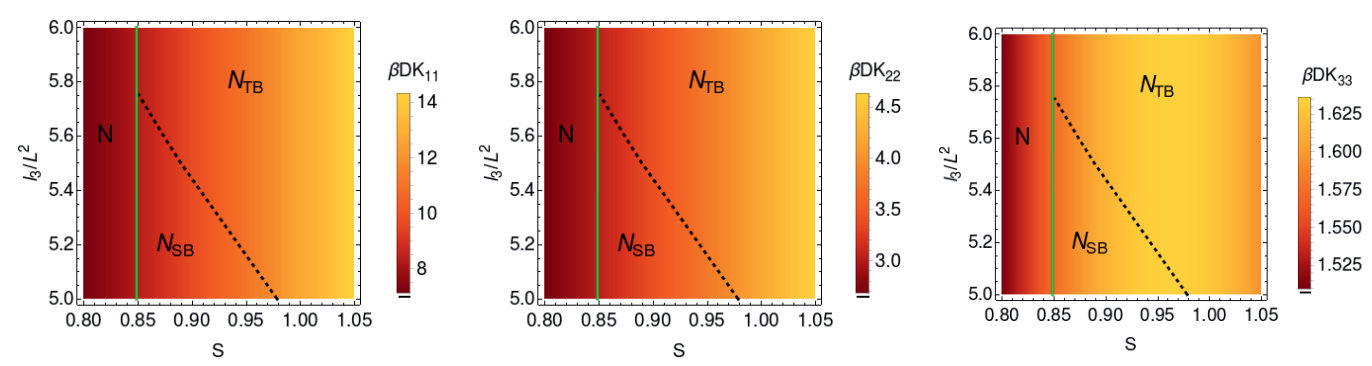

Figure 4.3: Same as Fig. 4.2, but now for $l_{1}=1.65 L^{2}$, and $l_{2}=0.854 L^{2}$. For $l_{3}<5.75 L^{2}$, the second-order $N-N_{\mathrm{SB}}$ transition is followed by a second-order $N_{\mathrm{SB}}-N_{\mathrm{TB}}$ transition.

\subsubsection{Phase Transitions between spatially modulated nematic phases}

The Oseen-Frank theories of Selinger and Dozov cannot describe phase transitions between periodically modulated nematic phases, since in these theories the elastic constants do not explicitly depend on control parameters, in contrast with the LdG framework. However, for $l_{3}=0$ in the case of the LdG theory described in the previous subsection, the elastic constants $K_{11}, K_{22}$ and $K_{33}$ depend on $S$ but the ratio $K_{11} / K_{22}$ is independent of $S$, and consequently the theory predicts only either an $N-N_{\mathrm{TB}}$ or an $N-N_{\mathrm{SB}}$ phase transition. In order to overcome this limitation we consider $l_{3} \neq 0$ in Eq. (4.3). This procedure not only removes the degeneracy between $K_{11}$ and $K_{33}$, but also allows the ratio $K_{11} / K_{22}$ to vary with particle concentration. Extending the computation of the previous subsection (for details see Appendix), we find that if $l_{3} \neq 0$, the ratio of the (negative) grand potential densities of the $N_{\mathrm{TB}}$ and $N_{\mathrm{SB}}$ phase close to the transition point is given by

$$
\frac{\Delta \Omega_{\mathrm{TB}}}{\Delta \Omega_{\mathrm{SB}}}=\frac{2 l_{1}+l_{2}}{4 l_{1}}+\frac{l_{3}}{8 l_{1}} S=\frac{K_{11}}{2 K_{22}}+\frac{3 l_{3}}{8 l_{1}} S
$$

where we have used that $K_{11}=S^{2}\left(2 l_{1}+l_{2}-S l_{3}\right)$ and $K_{22}=S^{2}\left(2 l_{1}\right)$. The critical scalar nematic order parameter at which $\Delta \Omega_{\mathrm{TB}}, \Delta \Omega_{\mathrm{SB}}$, and $K_{33}^{\text {eff }}$ vanish reads

$$
S_{c}=\frac{-4 l_{1}-2 l_{2}+S_{0} l_{3}}{2 l_{3}}+\sqrt{\frac{-9 S_{0} \lambda^{2}}{4 e_{2} l_{3}}+\frac{\left(4 l_{1}+2 l_{2}+S_{0} l_{3}\right)^{2}}{4 l_{3}^{2}}} .
$$

From the linear $S$-dependence of Eq. (4.27) one can deduce that, depending on the values of $l_{1}, l_{2}$ and $l_{3}$, either a second-order $N-N_{\text {TB }}$ phase transition occurs at $S_{c}$ followed by a secondorder $N_{\mathrm{TB}}-N_{\mathrm{SB}}$ phase transition at $S>S_{c}$, or a second-order $N-N_{\mathrm{SB}}$ transition occurs at $S_{c}$ succeeded by a second-order $N_{\mathrm{SB}}-N_{\mathrm{TB}}$ transition at $S>S_{c}$. To illustrate this, we map out the phase diagram as a function of the scalar nematic order parameter $S$ and coefficient $l_{3}$, for the coefficients $e_{2}=1, S_{0}=0.85, \kappa=0.1 L^{2}$ and $\lambda=0.1 L$. In Fig. 4.2, we display the resulting phase diagram three times with the background color denoting the values of the splay, twist, bend elastic constants as indicated by the color bar. The $I-N$ transition occurs at a nematic order parameter value of $S_{I N}=0.7922$. The green line in Fig. 4.2 corresponds to the set of 

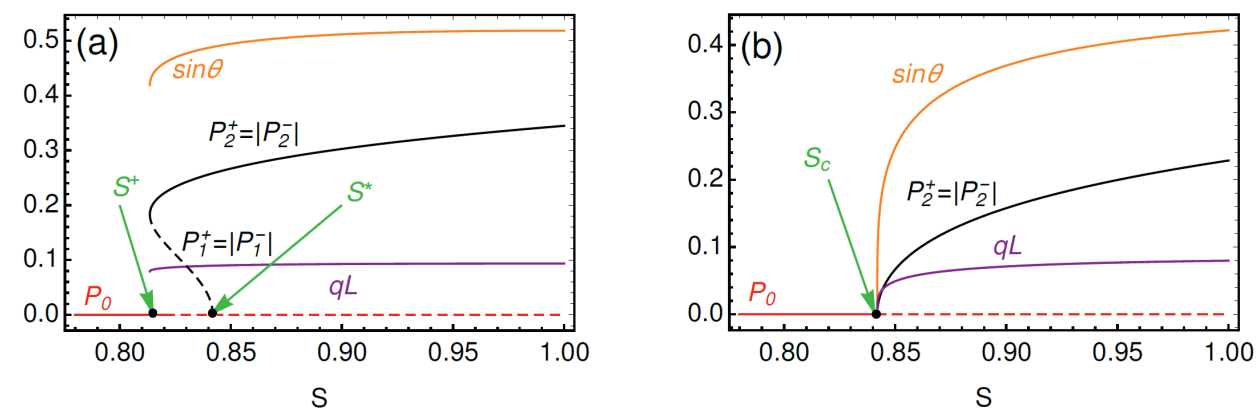

Figure 4.4: Amplitude of the polar vector $P$ and the equilibrium wave number $q$, and tilt angle $\theta$ as given by Eqs. (4.29), (4.12) and (4.13), respectively, as a function of the scalar order parameter $S$ for the coefficients $e_{2}=1, S_{0}=0.85, \lambda=0.08 L, \kappa=0.1 L^{2}, e_{6}=10$ and $e_{4}=-1(a)$ or $e_{4}=1(b)$. The uniaxial $N$ solution $P_{0}(S)=0$ is represented by a red full line when it is stable, and by a red dashed line when it is metastable. The $N_{\mathrm{TB}}$ solution $P_{2}^{ \pm}(S)$ is represented by a black full line, while the metastable solutions $P_{1}^{ \pm}(S)$ are represented by black dashed lines. The equilibrium $q$ and $\theta$ are represented in orange and violet, respectively. In $(a)$ the spinodal of the $N_{\mathrm{TB}}$ phase with respect to the uniaxial $N$ phase is at $S^{+}=0.826$, while the spinodal of the uniaxial $N$ phase with respect to the $N_{\text {TB }}$ phase is at $S^{*}=0.847$. In $(b)$ the second-order $N-N_{\text {TB }}$ transition occurs at $S_{c}=0.847$. We observe that $S^{*}=S_{c}$, i.e. the nematic spinodal of a first-order $N-N_{\mathrm{TB}}$ phase transition becomes the transition point if the $N-N_{\text {TB }}$ transition is second-order.

points where the renormalized $K_{33}^{\text {eff }}$ vanishes and hence the uniaxial $N$ phase becomes unstable with respect to the spatially modulated phases. For $l_{3}>-1.0 L^{2}$ and $l_{3}<-1.25 L^{2}$ only a second-order $N-N_{\mathrm{TB}}$ and a $N-N_{\mathrm{SB}}$ phase transition occur, respectively, as a function of $S$. For $-1.25 L^{2} \leq l_{3} \leq-1.0 L^{2}$, instead, the $N-N_{\mathrm{TB}}$ phase transition is followed by a second-order $N_{\mathrm{TB}}-N_{\mathrm{SB}}$ phase transition.

To map out the phase diagram of Fig. 4.2 , we have used the coefficients $l_{1}=0.165 L^{2}$ and $l_{2}=0.854 L^{2}$, obtained from a fit to the results from Onsager theory of hard rods, as stated in Section 4.3. For a different choice, $l_{1}=1.65 L^{2}$ and $l_{2}=0.854 L^{2}$, the phase sequence of $N-N_{\mathrm{TB}}-N_{\mathrm{SB}}$ can be replaced by a $N-N_{\mathrm{SB}}-N_{\mathrm{TB}}$ phase sequence, as shown in Fig. 4.3. Again, the $I-N$ phase transition occurs at $S_{I N}=0.7922$, the green line corresponds to the set of points where the uniaxial $N$ phase becomes unstable with respect to the spatially modulated phases, and the background color denotes the values of $K_{11}, K_{22}$, and $K_{33}$. We clearly observe from Fig. 4.3 that for $l_{3}<5.75 L^{2}$, the second-order $N-N_{\mathrm{SB}}$ phase transition is followed by a second-order $N_{\mathrm{SB}}-N_{\mathrm{TB}}$ transition.

\subsection{First-order $\mathrm{N}-\mathrm{N}_{\mathrm{TB}}$ phase transition}

We now consider the LdG theory of Section 4.2 with $e_{6}>0$ and allow $e_{4}$ to change sign in order to describe the first-order $N-N_{\text {TB }}$ phase transition recently found in Onsager theory and simulations $[64,65]$. For the sake of simplicity we set $l_{3}=0$ corresponding to a degenerate splay and bend elastic constant. We again start by inserting the nematic director $\hat{\mathbf{n}}_{\mathrm{TB}}(z)$ into the nematic tensor order parameter Eq. (4.4) and by inserting the resulting $\mathbf{Q}(z)$ together with 

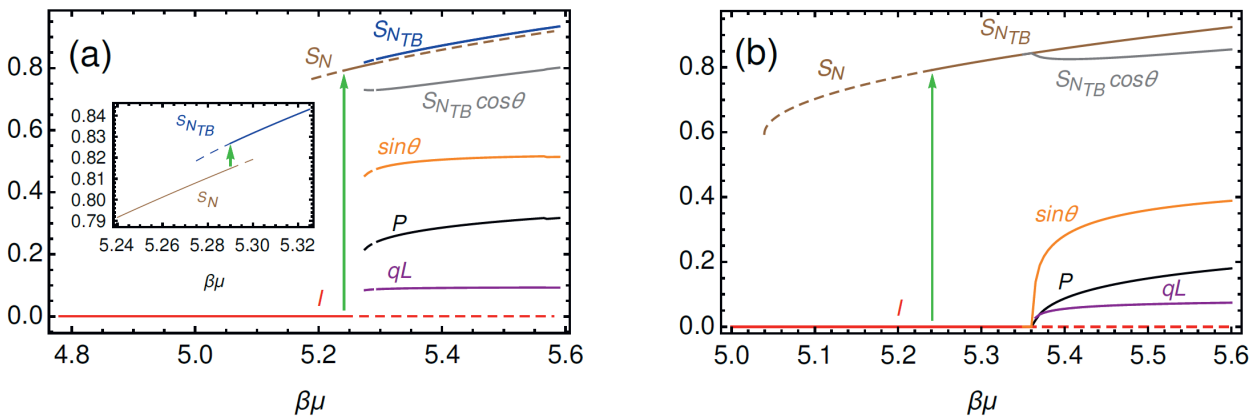

Figure 4.5: Scalar nematic order parameter (brown and blue) and amplitude of the polarity $P$ (black) as a function of the chemical potential $\beta \mu$, for the coefficients $e_{2}=1, \lambda=0.08 L, \kappa=0.1 L^{2}, e_{6}=$ $10, S_{0}=0.85$, and $e_{4}=-1(a)$ or $e_{4}=1(b)$. The equilibrium $q$ and $\theta$ are represented in orange and violet, respectively. In $(a)$ the "Onsager"-type first-order $I-N$ transition at $\beta \mu_{I N}=5.241$ is followed by a weakly first-order $N-N_{\mathrm{TB}}$ transition at $\beta \mu_{N N_{\mathrm{TB}}}=5.293$. In $(b)$ the "Onsager"-type first-order $I-N$ transition is instead followed by a continuous second-order $N-N_{\mathrm{TB}}$ transition at $\beta \mu_{N N_{\mathrm{TB}}}=5.36$.
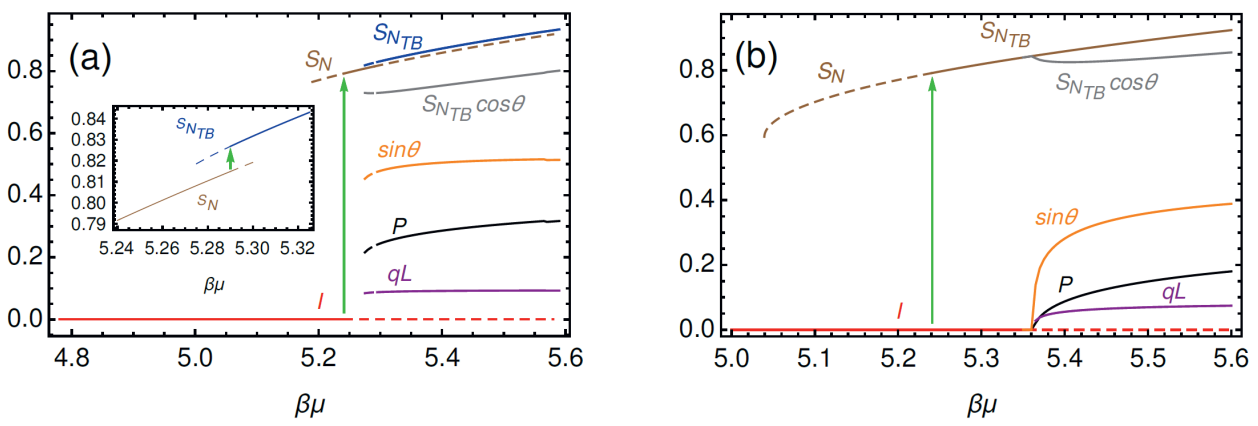

Figure 4.6: Scalar nematic order parameter (brown and blue) and amplitude of the polarity $P$ (black) as a function of the particle concentration $c=B_{2} \rho$, for the coefficients $e_{2}=1, \lambda=0.08 L, \kappa=$ $0.1 L^{2}, e_{6}=10, S_{0}=0.85$, and $e_{4}=-1(a)$ or $e_{4}=1(b)$. The equilibrium $q$ and $\theta$ are represented in orange and violet, respectively. In $(a)$ the "Onsager"-type first-order $I-N$ transition with coexisting concentrations $c_{I}=3.290$ and $c_{N}=4.191$, is followed by a weakly first-order $N-N_{\mathrm{TB}}$ transition with coexisting concentrations $c_{N}=4.265$ and $c_{N_{\mathrm{TB}}}=4.295$, i.e. a density jump on the order of $1 \%$. The inset shows a zoom of the discontinuous jump in the scalar order parameter at the $N-N_{\mathrm{TB}}$ transition. In (b) the "Onsager"-type first-order $I-N$ transition is instead followed by a continuous second-order $N-N_{\mathrm{TB}}$ transition at $c_{N}=c_{N_{\mathrm{TB}}}=4.36$. 

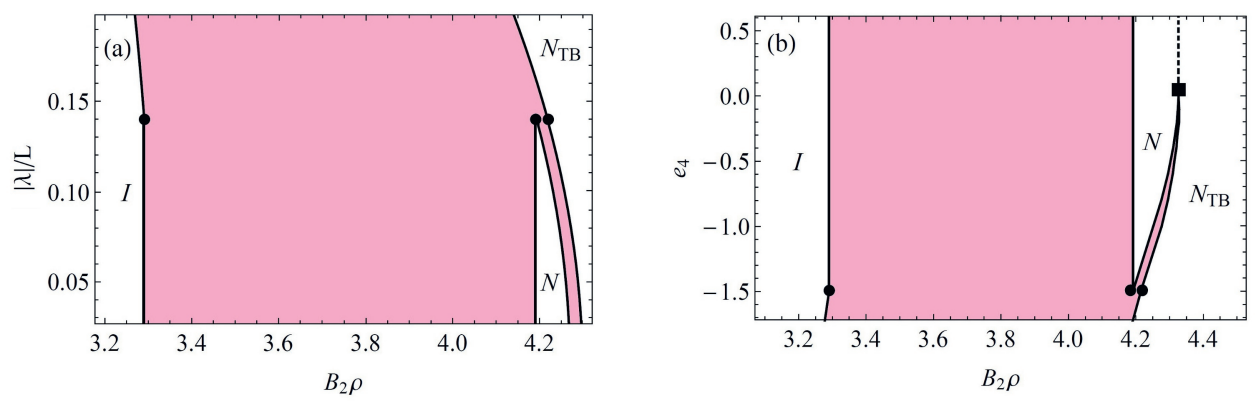

Figure 4.7: (a) Phase diagram as a function of the particle concentration $c=B_{2} \rho$ and the modulus of the flexoelectric coupling coefficient $|\lambda| / L$, for the coefficients $e_{2}=1, S_{0}=0.85, e_{4}=-1, \kappa=0.1 L^{2}$ and $e_{6}=10$. For a weak flexoelectric coupling $(|\lambda|<0.14 L)$, an "Onsager"-type first-order $I-N$ transition is followed by a weakly first-order $N-N_{\mathrm{TB}}$ transition while for strong coupling $(|\lambda|>0.14 L)$, a direct strongly first-order $I-N_{\mathrm{TB}}$ transition occurs. A $I-N-N_{\mathrm{TB}}$ triple point stabilizes at $|\lambda|=0.14 L$. $(b)$ Phase diagram as a function of the particle concentration $c=B_{2} \rho$ and the coefficient $e_{4}$, for the coefficients $e_{2}=1, \lambda=0.08 L, \kappa=0.1 L^{2}, e_{6}=10$ and $S_{0}=0.85$. For $-1.5<e_{4}<0$, the "Onsager"type first-order $I-N$ transition is followed by a weakly first-order $N-N_{\mathrm{TB}}$ phase transition while a strongly first-order $I-N_{\mathrm{TB}}$ transition occurs for $e_{4}<-1.5$. A $I-N-N_{\mathrm{TB}}$ triple point stabilizes at $e_{4}=-1.5$. The line of first-order $N-N_{\mathrm{TB}}$ transitions ends in a tricritical point $\left(c=4.3, e_{4}=0\right)$, from where it continues as a second-order $N-N_{\mathrm{TB}}$ transition. In $(a)$ as well as in $(b)$ the circles indicate triple points, the square indicates the tricritical point, the pink regions represent coexistence regions, and the dashed line indicates a second-order transition. 
$\mathbf{P}_{\mathrm{TB}}(z)$ in the grand potential Eq. (4.1). Minimizing the obtained grand potential with respect to the wave number $q$ and the tilt angle $\theta$, we find the expressions (4.12) and (4.13) already found in Section 4.4. We perform a stability analysis inserting these expressions into the grand potential Eq. (4.1) and minimize the resulting $\Omega$ with respect to $S$ and $P$ at fixed $\mu$, i.e. we solve the Euler-Lagrange equations $\partial \Delta \Omega / \partial S=0$ and $\partial \Delta \Omega / \partial P=0$. Analytically solving this system is cumbersome, since the two equations take the form of polynomials of third and fifth order, respectively, with a nonzero constant term. For this reason we directly minimize the grand potential $\Omega$ using a simulated annealing algorithm [70]. In this way we obtain $S$ and $P$ as a function of $\mu$. Note that in contrast with the situation discussed in Section 4.4 we cannot perform a stability analysis by minimizing $\Omega$ with respect to $P$ at fixed $S$, i.e. by solving the Euler-Lagrange equation $\partial \Delta \Omega / \partial P=0$. A jump in $S$ is expected at a first-order $N-N_{\mathrm{TB}}$ phase transition, and while we know the expression of $S_{N}$ as a function of $\mu$, we do not know $S_{N_{\mathrm{TB}}}$ as a function of $\mu$ analytically. Nevertheless, valuable insight can yet be obtained from the expression for $P$ as a function of $S$. For example, expressions can be derived for the spinodal of the uniaxial $N$ phase with respect to $N_{\mathrm{TB}}$, and for the spinodal of the $N_{\mathrm{TB}}$ phase with respect to the uniaxial $N$ phase. Hence, the limits of stability of the $N$ and $N_{\text {TB }}$ phases are analytically known. For small $P$ the solutions of the Euler-Lagrange equation $\partial \Delta \Omega / \partial P=0$ are given by

$$
\begin{aligned}
& P_{0}(S)=0 \\
& P_{1}^{ \pm}(S)= \pm \sqrt{\frac{-16 e_{4}-\sqrt{\gamma(S)}}{48 e_{6}}} \\
& P_{2}^{ \pm}(S)= \pm \sqrt{\frac{-16 e_{4}+\sqrt{\gamma(S)}}{48 e_{6}}}
\end{aligned}
$$

with $\gamma(S)=256 e_{4}^{2}-96 e_{6}\left(8 e_{2}-8 S e_{2} / S_{0}-9 \lambda^{2} /\left(2 l_{1}+l_{2}\right)\right)$. The solution $P_{0}(S)$ corresponds to the uniaxial $N$ phase. If $e_{4}<0$, we find for increasing $S$ a jump from the uniaxial $N$ phase corresponding to $P_{0}(S)$ to the two (equivalent) solutions $P_{2}^{ \pm}(S)$, while the solutions $P_{1}^{ \pm}(S)$ are always metastable. The solutions $P_{2}^{ \pm}(S)$ represent the $N_{\mathrm{TB}}$ phase with equilibrium wave vector $q$ and equilibrium angle $\theta$ given by Eqs. (4.12) and (4.13), respectively. The spinodal of the $N_{\text {TB }}$ phase with respect to the uniaxial $N$ phase, given by the condition $\partial^{2} \Delta \Omega /\left.\partial^{2} P\right|_{P=P_{2}^{ \pm}}=0$, is at

$$
S^{+}=S_{0}\left(1-\frac{9 \lambda^{2}}{8 e_{2}\left(2 l_{1}+l_{2}\right)}-\frac{e_{4}^{2}}{4 e_{2} e_{6}}\right),
$$

while the spinodal of the uniaxial $N$ phase with respect to the $N_{\text {TB }}$ phase, given by the condition $\partial^{2} \Delta \Omega /\left.\partial^{2} P\right|_{P=P_{0}}=0$, is at

$$
S^{*}=S_{0}\left(1-\frac{9 \lambda^{2}}{8 e_{2}\left(2 l_{1}+l_{2}\right)}\right) .
$$

If instead $e_{4} \geq 0$, we find a second-order phase transition from the $N$ phase with $P_{0}(S)=0$ to the $N_{\mathrm{TB}}$ phase with $P_{2}^{ \pm}(S)$ at $S_{c}=S_{0}\left(1-9 \lambda^{2} /\left(8 e_{2}\left(2 l_{1}+l_{2}\right)\right)\right)$, while the solutions $P_{1}^{ \pm}(S)$ are imaginary and hence unphysical. Note that the nematic spinodal of the first-order $N$ $N_{\text {TB }}$ phase transition coincides with the transition point of the second-order $N-N_{\mathrm{TB}}$ transition. Furthermore the transition point of the second-order $N-N_{\text {TB }}$ transition found in this Section coincides with the one found in Section 4.4.

Exemplarily, we show the analytical solutions for the amplitude of the polar vector $P$ (see (4.29)) and the equilibrium wave vector $q$ denoted in orange and tilt angle $\theta$ in violet as given 
by Eqs. (4.12) and (4.13) in Fig. 4.4 as a function of the scalar nematic order parameter $S$ for the coefficients $e_{2}=1, S_{0}=0.85, \lambda=0.08 L, \kappa=0.1 L^{2}, e_{6}=10$ and $e_{4}=-1$ (Fig. 4.4(a)) and $e_{4}=1$ (Fig. 4.4(b)). The uniaxial $N$ solution $P_{0}(S)=0$ is represented by a red full line when it is stable, and by a red dashed line when it is metastable. The $N_{\mathrm{TB}}$ solution $P_{2}^{ \pm}(S)$ is represented by a black full line, while the metastable solutions $P_{1}^{ \pm}(S)$ are represented by black dashed lines. We find that in $(a)$ the spinodal of the $N_{\mathrm{TB}}$ phase with respect to the uniaxial $N$ phase is at $S^{+}=0.826$, while the spinodal of the uniaxial $N$ phase with respect to the $N_{\mathrm{TB}}$ phase is at $S^{*}=0.847$. In $(b)$ the second-order $N-N_{\text {TB }}$ transition occurs at $S_{c}=0.847$. We note that $S^{*}=S_{c}$, i.e. the nematic spinodal of a first-order $N-N_{\mathrm{TB}}$ phase transition becomes the transition point if the $N-N_{\mathrm{TB}}$ transition is second-order. In addition, we find that in the case of a second-order $N-N_{\text {TB }}$ phase transition, the equilibrium $q$ and $\theta$ tend to zero at the transition, in agreement with Refs. [53,139].

The solutions $S(\mu)$ and $P(\mu)$ as obtained by directly minimizing $\Omega$ are plotted in Fig. 4.5. In addition, we plot $\theta, q$ and $S \cos \theta$ as a function of $\mu$ in the same figure. The dependence on chemical potential in Fig. 4.5 is then converted to concentration in Fig. 4.6. If $e_{4}=-1$, upon increasing $\mu$ we find the "Onsager"-type first-order $I-N$ phase transition described in Section 4.3 , followed by a weakly first-order $N-N_{\mathrm{TB}}$ phase transition at $\beta \mu_{N N_{\mathrm{TB}}}=5.293$, where the nematic order parameter jumps from $S_{N}=0.814$ to $S_{N_{\mathrm{TB}}}=0.836$. The coexisting densities are $c_{N}=4.265$ and $c_{N_{\text {Тв }}}=4.295$, i.e. a density jump on the order of $1 \%$. Interestingly, we also find jumps in $P, \theta, q$ and $S_{N} \cos \theta$, which is to be contrasted with a second-order $N$ $N_{\text {TB }}$ phase transition. For instance, upon increasing the chemical potential $\mu$ for the parameter $e_{4}=1$, we find an "Onsager"-type $I-N$ phase transition and subsequently a second-order $N-N_{\mathrm{TB}}$ transition at $\beta \mu_{N N_{\mathrm{TB}}}=5.36$, where $S_{N}\left(\mu_{N N_{\mathrm{TB}}}\right)=S_{N_{\mathrm{TB}}}\left(\mu_{N N_{\mathrm{TB}}}\right)=0.847, c_{N}=c_{N_{\mathrm{TB}}}=4.36$, and $P=\theta=q=0$, so no jumps at all.

Finally, we map out two phase diagrams of Figs. $4.7(a)$ and $4.7(b)$. In Fig. $4.7(a)$ we plot the phase diagram as a function of the particle concentration $c=B_{2} \rho$ and the modulus of the flexoelectric coupling coefficient $|\lambda|$ for the same coefficients as above, i.e. $e_{2}=1, S_{0}=$ $0.85, e_{4}=-1, \kappa=0.1 L^{2}$ and $e_{6}=10$. The pink regions represent two-phase coexistence regions. At $|\lambda|<0.14 L$, the phase diagram features the "Onsager"-type first-order $I-N$ transition followed by a weakly first-order $N-N_{\mathrm{TB}}$ transition at higher densities. At $|\lambda|>0.14 L$, however, we find a direct first-order $I-N_{\mathrm{TB}}$ transition of which the coexisting densities decrease with increasing $|\lambda|$. The two regimes are separated by an $I-N-N_{\mathrm{TB}}$ triple point at $|\lambda|=0.14 L$. It is important to observe that the $I-N_{\mathrm{TB}}$ transition remains always first-order such that we never find a second-order $I-N_{\mathrm{TB}}$ transition. Moreover at $\lambda=0$ a first-order phase transition is found from a uniaxial $N$ phase to an $N_{\text {TB }}$ phase with an infinite pitch, i.e. a polar nematic phase. In Fig. 4.7(b), instead, we build a phase diagram as a function of particle concentration $c=B_{2} \rho$ and coefficient $e_{4}$ of the $|P|^{4}$ term that we allow to be negative because of the presence of a stabilising (positive) $|P|^{6}$ term in the grand potential. We fix the other coefficients to the values as employed above, i.e. $e_{1}=1, \lambda=0.08 L, \kappa=0.1 L^{2}, e_{6}=10$ and $S_{0}=0.85$. Again the pink regions represent bulk coexistence regions. We note that we have chosen a value of $\lambda$ such that the $I-N$ transition is followed by an $N-N_{\mathrm{TB}}$ transition at $e_{4}=-1$ according to Fig. $4.7(a)$. For $-1.5<e_{4}<0$, we find the "Onsager"-type first-order $I-N$ transition followed by a weakly first-order $N-N_{\mathrm{TB}}$ transition. We observe, however, a direct strongly first-order $I-N_{\mathrm{TB}}$ transition for $e_{4}<-1.5$ and an $I-N-N_{\mathrm{TB}}$ triple point at $e_{4}=-1.5$. Furthermore, we find that the line of first-order $N-N_{\mathrm{TB}}$ transitions ends in a tricritical point at $\left(c=4.3, e_{4}=0\right)$. At $e_{4}>0$ the $N-N_{\text {TB }}$ transition ceases to be first-order and becomes second-order as illustrated by the dashed line. 
As a final observation, we mention that the formalism introduced in this Section should also hold for the $N_{\mathrm{SB}}$ phase. Despite reasonable efforts, but not exhaustive, we did not find a set of coefficients for which the $N_{\mathrm{SB}}$ phase is more stable than the $N_{\mathrm{TB}}$ one. However, since in simulations and Onsager theory only an $N-N_{\text {TB }}$ phase transition was found, we focused on the latter one. Very interestingly, the nematic spinodal of such a putative $N-N_{\mathrm{SB}}$ transition would coincide with the nematic spinodal of the $N-N_{\mathrm{TB}}$ phase found here. Furthermore, by breaking the degeneracy between $K_{11}$ and $K_{33}$, i.e. by setting $l_{3} \neq 0$ in Eq. (4.3), the first-order $N-N_{\mathrm{TB}}$ phase transition described in this section could be followed by a first-order $N_{\mathrm{TB}}-N_{\mathrm{SB}}$ phase transition.

\subsection{Renormalized elastic constants}

The flexoelectric coupling term $-\lambda P_{\alpha} \partial_{\beta} Q_{\beta \alpha}$ of Eq. (4.6) affects the elastic response of polar nematic phases, which translates into renormalized elastic constants. In order to quantify the renormalization of the elastic constants we follow the procedure of Refs. [49,53]. First we solve the Euler-Lagrange equation $\partial\left(\beta B_{2} \omega_{P}\right) / \partial P_{\rho}=0$. By using Eq. (4.4) for the tensor order parameter $\mathbf{Q}$, the fact that $\mathbf{P}$ and $\hat{\mathbf{n}}$ are perpendicular, i.e. $P_{\alpha} n_{\alpha}=0$, and that $P_{\alpha} P_{\alpha}=0$ in the uniaxial $N$ phase, we find

$$
P_{\rho}=\frac{\lambda S_{0}}{2 e_{2}\left(S_{0}-S\right)} \partial_{\gamma} Q_{\rho \gamma}
$$

which upon insertion into Eq. (4.6), with $l_{3}=0$ for convenience, yields

$$
\beta B_{2} \omega_{P}=-\frac{\lambda^{2} S_{0}\left(S_{0}-2 S\right)}{4 e_{2}\left(S_{0}-S\right)^{2}}\left(\partial_{\gamma} Q_{\gamma \alpha}\right)\left(\partial_{\beta} Q_{\beta \alpha}\right)+\frac{\lambda^{2} S_{0}}{2 e_{2}\left(S_{0}-S\right)^{2}}\left(\partial_{\gamma} Q_{\gamma \alpha}\right) Q_{\alpha \beta}\left(\partial_{\xi} Q_{\xi \beta}\right)
$$

In the final step we neglected the terms $e_{4} P_{\alpha} P_{\alpha} P_{\beta} P_{\beta}, \kappa\left(\partial_{\alpha} P_{\beta}\right)\left(\partial_{\alpha} P_{\beta}\right)$ and $e_{6} P_{\alpha} P_{\alpha} P_{\beta} P_{\beta} P_{\gamma} P_{\gamma}$ in Eq. (4.6) because they do not contribute to the linear elasticity as they contain derivatives of the nematic director of order higher than two. It follows that the form of the renormalized elastic constant is the same for second-order transitions $\left(e_{4}>0, e_{6} \geq 0\right)$ as well as for first-order ones $\left(e_{4}<0, e_{6}>0\right)$. Using that $n_{i}\left(\partial_{j} n_{i}\right)=\left(\partial_{j} n_{i}\right) n_{i}=0$ and that

$$
\begin{aligned}
\left(\partial_{\alpha} n_{\beta}\right)\left(\partial_{\alpha} n_{\beta}\right) & =(\nabla \cdot \hat{\mathbf{n}})^{2}+[\hat{\mathbf{n}} \cdot(\nabla \times \hat{\mathbf{n}})]^{2}+|\hat{\mathbf{n}} \times(\nabla \times \hat{\mathbf{n}})|^{2} \\
& -\nabla \cdot[\hat{\mathbf{n}}(\nabla \cdot \hat{\mathbf{n}})+\hat{\mathbf{n}} \times(\nabla \times \hat{\mathbf{n}})],
\end{aligned}
$$

we find

$$
\begin{aligned}
\beta B_{2}\left(\omega_{e}+\omega_{P}\right) & =\left[l_{1}+\frac{1}{2} l_{2}-\frac{S_{0}-4 S}{S_{0}-S} \xi(S)\right] S^{2}(\nabla \cdot \hat{\mathbf{n}})^{2}+\left[l_{1}+\frac{1}{2} l_{2}-\xi(S)\right] S^{2}|\hat{\mathbf{n}} \times(\nabla \times \hat{\mathbf{n}})|^{2} \\
& +\frac{1}{3}\left[2 l_{2}+\frac{10 S-3 S_{0}}{S_{0}-S} \xi(S)\right] S(\nabla \cdot \hat{\mathbf{n}})(\nabla S \cdot \hat{\mathbf{n}})+\left[\frac{1}{6} l_{2}-\frac{S_{0}-3 S}{S_{0}-S} \xi(S)\right](\nabla S \cdot \hat{\mathbf{n}})^{2} \\
& +\left[\frac{1}{3} l_{2}-\xi(S)\right]|\nabla S|^{2}+l_{1} S^{2}\left\{[\hat{\mathbf{n}} \cdot(\nabla \times \hat{\mathbf{n}})]^{2}-\nabla \cdot[\hat{\mathbf{n}}(\nabla \cdot \hat{\mathbf{n}})+\hat{\mathbf{n}} \times(\nabla \times \hat{\mathbf{n}})]\right\},
\end{aligned}
$$




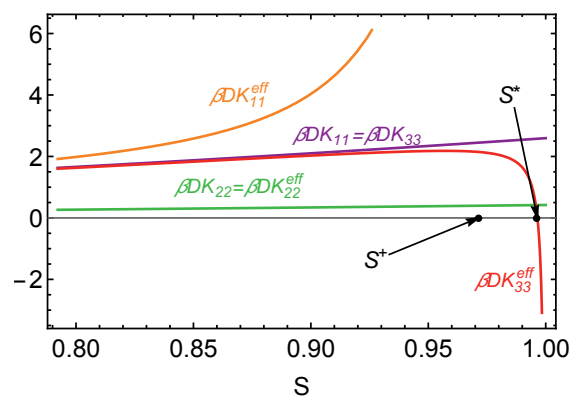

Figure 4.8: Bare $\left(K_{11}, K_{22}, K_{33}\right)$ and renormalized $\left(K_{11}^{\text {eff }}, K_{22}^{\text {eff }}, K_{33}^{\text {eff }}\right)$ splay, twist and bend elastic constants as a function of the scalar nematic order parameter $S$, for the fixed values of the coefficients $e_{2}=1, S_{0}=1, \lambda=0.08 L$ and $e_{6}=10$. While $K_{11}^{\text {eff }}, K_{11}=K_{33}$ and $K_{22}^{\text {eff }}=K_{22}$ increase monotonically with $S, K_{33}^{\text {eff }}$ starts to decrease beyond a certain value of $S$, until it becomes zero at $S=S_{0}(1-$ $\left.9 \lambda^{2} /\left(8 e_{2}\left(2 l_{1}+l_{2}\right)\right)\right)$. This nematic order parameter value $S_{c}$ is where the $N-N_{\mathrm{TB}}$ transition occurs if it is second-order, while it is the spinodal $S^{*}$ of the $N$ phase with respect to the $N_{\text {TB }}$ one in the case of a first-order $N-N_{\mathrm{TB}}$ transition. $S^{+}$indicates the spinodal of the $N_{\mathrm{TB}}$ phase with respect to the uniaxial $N$ one.

with $\xi(S)=9 \lambda^{2} S_{0} /\left(16 e_{2}\left(S_{0}-S\right)\right)$. Comparing Eq. (4.35) with the Oseen-Frank elastic energy (4.5) gives us the renormalized splay $\left(K_{11}^{\mathrm{eff}}\right)$, twist $\left(K_{22}^{\mathrm{eff}}\right)$, and bend $\left(K_{33}^{\mathrm{eff}}\right)$ elastic constants

$$
\begin{aligned}
& \beta D K_{11}^{\mathrm{eff}}=\beta D K_{11}-\frac{8 S^{2}}{\pi L^{2}} \frac{S_{0}-4 S}{S_{0}-S} \xi(S), \\
& \beta D K_{22}^{\mathrm{eff}}=\beta D K_{22}, \\
& \beta D K_{33}^{\mathrm{eff}}=\beta D K_{33}-\frac{8 S^{2}}{\pi L^{2}} \xi(S),
\end{aligned}
$$

where $K_{11}, K_{22}$ and $K_{33}$ are the bare splay, twist and bend elastic constants discussed in Section 4.2. Eq. (4.36) directly reveals the flexoelectric coupling as the source of the renormalization, since $\lambda=0$ gives $\xi(S)=0$ and hence no renormalization at all. For $\lambda \neq 0$, we observe, however, that the renormalization procedure breaks the degeneracy between the renormalized splay and bend elastic constants, so even though $K_{11}=K_{33}$, we have nevertheless that $K_{11}^{\text {eff }} \neq K_{33}^{\text {eff }}$. The renormalization does not affect the twist elastic constant, for which $K_{22}=K_{22}^{\text {eff }}$. The elastic constants $K_{11}^{\text {eff }}, K_{22}^{\text {eff }}=K_{22}$ and $K_{11}=K_{33}$ increase monotonically with $S$, while the renormalized bend elastic constant $K_{33}^{\text {eff }}$ starts to decrease beyond a certain value of $S$ until it becomes zero at $S_{c}=S_{0}\left(1-9 \lambda^{2} /\left(8 e_{2}\left(2 l_{1}+l_{2}\right)\right)\right)$. As discussed in Sections 4.4 and 4.5 , the second-order $N-N_{\text {TB }}$ phase transition occurs at a nematic order parameter value $S_{c}$, whereas in the case of a first-order transition the spinodal of the $N$ phase with respect to the $N_{\mathrm{SB}}$ phase is located at $S_{c}$. As a consequence, our result is in agreement with Selinger's one in the case of a second-order $N-N_{\mathrm{TB}}$ transition, while in the case of a first-order transition we find that at the transition point both $K_{33}>0$ and $K_{33}^{\text {eff }}>0$. In the latter case $K_{33}^{\text {eff }}$ becomes zero at the nematic spinodal. The bare as well as the renormalized elastic constants are plotted as a function of the scalar order parameter $S$ in Fig. 4.8, for the coefficients $e_{2}=1, S_{0}=1, \lambda=0.08 L$ and $e_{6}=10$. The monotonic increase of $K_{11}^{\text {eff }}, K_{22}^{\text {eff }}=K_{22}$ and $K_{11}=K_{33}$ with $S$, together with the simultaneous softening of $K_{33}^{\text {eff }}$ can be observed in the figure. The picture is qualitatively the same for $l_{3} \neq 0$, with $K_{33}^{\text {eff }}$ vanishing at $S_{c}$ as given by Eq. (4.28). 


\subsection{Conclusions and discussion}

In this Chapter we have developed a phenomenological LdG theory for lyotropic suspensions of bent hard rods, using a $Q$-tensor expansion of the chemical-potential dependent grand potential. In addition, we introduce a bend flexoelectric term that couples the polarization and the divergence of the $Q$-tensor, to study the stability of uniaxial $(N)$, twist-bend $\left(N_{\mathrm{TB}}\right)$ and splaybend $\left(N_{\mathrm{SB}}\right)$ nematic phases of colloidal bent rods as a function of particle concentration. We first showed that our LdG theory can be mapped onto an Oseen-Frank theory. Subsequently, by breaking the degeneracy between the splay and bend elastic constants, we find that the LdG theory predicts either an $N-N_{\mathrm{TB}}-N_{\mathrm{SB}}$ or an $N-N_{\mathrm{SB}}-N_{\mathrm{TB}}$ phase sequence upon increasing the particle concentration. We have mapped out several phase diagrams as a function of the particle concentration that can be used as guidelines for experiments, simulations and microscopic theories. In addition, we have focused on the first-order $N-N_{\mathrm{TB}}$ phase transition. Our model is able to reproduce the discontinuous jumps associated with this transition, including the density jump and the discontinuities in the polarization and nematic order. Moreover, in contrast with the case of a second-order $N-N_{\text {TB }}$ phase transition where the bend elastic constant $K_{33}$ is positive while its renormalized version $K_{33}^{\text {eff }}$ vanishes, we have found that $K_{33}^{\text {eff }}$ vanishes at the nematic spinodal in the case of a first-order $N-N_{\text {TB }}$ transition, so that $K_{33}$ as well as $K_{33}^{\text {eff }}$ remain positive at the actual transition point. This finding appears to be general and could help in understanding the problem of the softening of the elastic constants in systems with spontaneous polar order and of the mechanism driving the onset of the spatially modulated phases in bent-core liquid crystals.

Finally, it is interesting to employ the LdG theory to study confinement effects of these spatially modulated nematic phases and to investigate the structure of the interface between a coexisting $N_{\mathrm{TB}}$ or $N_{\mathrm{SB}}$ phase with either an isotropic, uniaxial nematic, or with a substrate. We will postpone this to future work.

\subsection{Acknowledgements}

We acknowledge help by Dr. S. Paliwal with the numerical calculations and by A. Grau Carbonell in the realization of Fig. 4.1.

\subsection{Appendix A: Grand potential density of the $N_{\mathrm{TB}}$ and $N_{\mathrm{SB}}$ phases for $l_{3} \neq 0$.}

In order to compute the grand potential density of the $N_{\mathrm{TB}}$ phase, we insert

$$
\hat{\mathbf{n}}_{\mathrm{TB}}=(\sin \theta \cos (q z), \sin \theta \sin (q z), \cos \theta)
$$

in the tensor order parameter $Q_{\alpha \beta}(\mathbf{r})=\frac{3}{2} S(\mathbf{r})\left(n_{\alpha}(\mathbf{r}) n_{\beta}(\mathbf{r})-\frac{1}{3} \delta_{\alpha \beta}\right)$, and the resulting $\mathbf{Q}$, together with $\mathbf{P}_{\mathrm{TB}}=(P \sin (q z),-P \cos (q z), 0)$, in the grand potential given by Eq. (4.1). We minimize the obtained grand potential with respect to the wave number $q$ and the tilt angle $\theta$, respectively, and find

$$
q_{\mathrm{TB}}=\frac{3 \lambda \sin \left(2 \theta_{\mathrm{TB}}\right) S P}{8 \kappa P^{2}+4 S^{2}\left(2 l_{1}+l_{2}\right) \sin ^{2} \theta_{\mathrm{TB}}-4 l_{2} S^{2} \sin ^{4} \theta_{\mathrm{TB}}+2 S^{3} l_{3}\left(\sin ^{2} \theta_{\mathrm{TB}}-\sin ^{4} \theta_{\mathrm{TB}}\right)},
$$


and

$$
\sin ^{2} \theta_{\mathrm{TB}}=\frac{\kappa P^{2}}{S^{2} l_{1}}+\frac{\sqrt{\kappa P^{2}\left(\kappa P^{2}+S^{2} l_{1}\right)}}{S^{2} l_{1}} .
$$

Inserting (4.38) and (4.39) back into Eq. (4.1), and approximating for small $P$, the grand potential density in the $N_{\mathrm{TB}}$ phase is given by

$$
\begin{aligned}
\frac{\Delta \Omega_{\mathrm{TB}}}{V} & \equiv \frac{\Delta \Omega}{V}-\frac{\Delta \Omega_{N}}{V} \\
& =\left[\frac{e_{2}\left(S_{0}-S\right)}{S_{0}}-\frac{9 \lambda^{2}}{4\left(4 l_{1}+2 l_{2}+S l_{3}\right)}\right] P^{2}+\frac{18 \lambda^{2} \sqrt{\kappa S^{2} l_{1}}}{S^{2}\left(4 l_{1}+2 l_{2}+S l_{3}\right)^{2}}|P|^{3}+e_{4} P^{4}+O\left(P^{5}\right)
\end{aligned}
$$

where $\Delta \Omega_{N} / V=a S^{2}\left(\mu^{*}-\mu\right)-b S^{3}+d S^{4}$ is the grand-potential density of the $N$ phase.

Analogously, in order to compute the grand potential density of the $N_{\mathrm{SB}}$ phase, we insert the nematic director $\hat{\mathbf{n}}_{\mathrm{SB}}=(\sin (\theta \sin (q z)), 0, \cos (\theta \sin (q z)))$ into the tensor order parameter $Q_{\alpha \beta}(\mathbf{r})=\frac{3}{2} S(\mathbf{r})\left(n_{\alpha}(\mathbf{r}) n_{\beta}(\mathbf{r})-\frac{1}{3} \delta_{\alpha \beta}\right)$, and the resulting $\mathbf{Q}$, together with

$$
\mathbf{P}_{\mathrm{SB}}=\left(-P \cos (q z) \cos (\theta \sin (q z)), 0, \frac{1}{2} P \cos (q z) \sin (2 \theta \sin (q z))\right),
$$

into the grand potential given by Eq. (4.1), and average it over a full period $2 \pi / q$. We then minimize the obtained grand potential with respect to the wave number $q$ and the tilt angle $\theta$, respectively, and find

$$
q_{\mathrm{SB}}=\frac{3 \lambda \theta_{\mathrm{SB}}\left(\theta_{\mathrm{SB}}^{2}-8\right) P S}{8\left(4+3 \theta_{\mathrm{SB}}^{2}\right) \kappa P^{2}+16 S^{2}\left(2 l_{1}+l_{2}+S l_{3}\right) \theta_{\mathrm{SB}}^{2}},
$$

and

$$
\theta_{\mathrm{SB}}^{2}=\frac{16 \kappa P^{2}}{3 \kappa P^{2}+\sqrt{\kappa P^{2}\left(57 \kappa P^{2}+16 S^{2}\left(4 l_{1}+2 l_{2}+S l_{3}\right)\right)}} .
$$

Inserting Eqs. (4.42) and (4.43) back into Eq. (4.1) and approximating for small $P$, the grand potential density of the $N_{\mathrm{SB}}$ phase reads

$$
\begin{aligned}
\frac{\Delta \Omega_{S B}}{V} & \equiv \frac{\Delta \Omega}{V}-\frac{\Delta \Omega_{N}}{V} \\
& =\left[\frac{e_{2}\left(S_{0}-S\right)}{2 S_{0}}-\frac{9 \lambda^{2}}{8\left(4 l_{1}+2 l_{2}+S l_{3}\right)}\right] P^{2}-\frac{9 \lambda^{2} \sqrt{\kappa S^{2}\left(4 l_{1}+2 l_{2}+S l_{3}\right)}}{4 S^{2}\left(4 l_{1}+2 l_{2}+S l_{3}\right)^{2}}|P|^{3} \\
& +\frac{3}{8} e_{4} P^{4}+O\left(P^{5}\right)
\end{aligned}
$$

where $\Delta \Omega_{N} / V=a S^{2}\left(\mu^{*}-\mu\right)-b S^{3}+d S^{4}$ is again the grand potential density of the $N$ phase.

We observe that for small $P$ both $\Delta \Omega_{\mathrm{TB}}$ and $\Delta \Omega_{\mathrm{SB}}$ vanish at the critical point $S_{c}$ given by Eq. (4.28). Close to this point we can assume $P_{\mathrm{TB}} \ll 18 \lambda^{2} \sqrt{\kappa S^{2} l_{1}} /\left(e_{4} S^{2}\left(4 l_{1}+2 l_{2}+\right.\right.$ $\left.S l_{3}\right)^{2}$ ) and $P_{\mathrm{SB}} \ll 6 \lambda^{2} \sqrt{\kappa S^{2}\left(4 l_{1}+2 l_{2}+S l_{3}\right)} /\left(e_{4} S^{2}\left(4 l_{1}+2 l_{2}+S l_{3}\right)^{2}\right)$, such that the cubic terms dominate over the quartic terms in Eqs. (4.40) and (4.44). Solving the Euler-Lagrange equations $\partial\left(\Delta \Omega_{\mathrm{TB}} / V\right) / \partial P=0$ and $\partial\left(\Delta \Omega_{\mathrm{SB}} / V\right) / \partial P=0$, we find

$$
P_{\mathrm{TB}}=\frac{\left(9 \lambda^{2} S_{0}-4 e_{2}\left(S_{0}-S\right)\left(4 l_{1}+2 l_{2}+S l_{3}\right)\right)\left(4 l_{1}+2 l_{2}+S l_{3}\right) S^{2}}{108 \lambda^{2} l_{1} S_{0} \sqrt{\kappa S^{2} l_{1}}},
$$




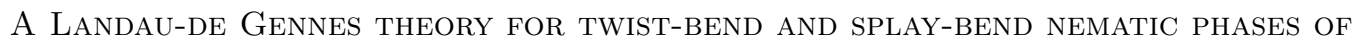
COLLOIDAL SUSPENSIONS OF BENT RODS

and

$$
P_{\mathrm{SB}}=\frac{\left(9 \lambda^{2} S_{0}-4 e_{2}\left(S_{0}-S\right)\left(4 l_{1}+2 l_{2}+S l_{3}\right)\right) \sqrt{\kappa S^{2}\left(4 l_{1}+2 l_{2}+S l_{3}\right)}}{27 \lambda^{2} \kappa S_{0}},
$$

respectively. Inserting these back in Eqs. (4.40) and (4.44) gives close to the transition

$$
\frac{\Delta \Omega_{\mathrm{TB}}}{V}=\frac{\left(-9 \lambda^{2} S_{0}+4 e_{2}\left(S_{0}-S\right)\left(4 l_{1}+2 l_{2}+S l_{3}\right)\right)^{3}\left(4 l_{1}+2 l_{2}+S l_{3}\right) S^{2}}{46656 \kappa \lambda^{2} l_{1} S_{0}^{3}}
$$

and

$$
\frac{\Delta \Omega_{\mathrm{SB}}}{V}=\frac{\left(-9 \lambda^{2} S_{0}+4 e_{2}\left(S_{0}-S\right)\left(4 l_{1}+2 l_{2}+S l_{3}\right)\right)^{3} S^{2}}{5832 \kappa \lambda^{2} S_{0}^{3}},
$$

respectively. The ratio between the grand potential densities (4.47) and (4.48) is then given by Eq. (4.27). 



\section{Evidence of density modulations in the splay-bend nematic phase of colloidal suspensions of bent rods}

The twist-bend nematic and splay-bend nematic phases were theoretically predicted by Meyer in 1976 and by Dozov in 2001 for bent-core particles that favor spontaneous bend deformations in the nematic director field. Here, we investigate the repercussion of the splay distortions in the nematic director field on the density in splay-bend nematic phases. To this aim, we employ the Landau-de Gennes theory introduced in Chapter 4 of this thesis, that naturally involves a coupling between the nematic order parameter $S$ and the particle density $\rho$. We find a spatially modulating $S(z)$ and hence a one-dimensional density modulation in the splay-bend phase. As a consequence, the splay-bend phase has actually the key characteristics of a smectic rather than a nematic phase. By contrast we find that $S$ does not vary in space in the twist-bend phase, which is therefore a true nematic phase. Our results are in agreement with recent simulation findings. 


\subsection{Introduction}

During the past decades, much research in liquid crystal science has focused on mesophases formed by thermotropic mesogens with a "banana-like" or bent-core shape, that favor spontaneous bend deformations in the nematic director field [49]. As a pure bend deformation cannot uniformly fill the 3D space, Meyer [50] and later independently Dozov [51] argued that the local bend deformations have to be accompanied by either a twist or splay deformation. In the former case they theoretically postulated the stabilization of a spatially modulated twist-bend nematic $\left(N_{\mathrm{TB}}\right)$ phase, displaying a heliconical variation with bend and twist deformation in the molecular orientation. In the latter, they predicted the stabilization of a spatially modulated splay-bend nematic $\left(N_{\mathrm{SB}}\right)$ phase, characterized by alternating domains of splay and bend.

After its theoretical prediction, the $N_{\text {TB }}$ phase was experimentally observed in several molecular systems [159-163], and has meanwhile become well-established as a new type of orientationally ordered fluid phase. By contrast, the $N_{\mathrm{SB}}$ phase turns out to be elusive in experiments. However, more recently, simulations have revealed that an $N_{\mathrm{SB}}$ phase can be stable in systems of hard bent spherocylinders, provided the smectic ( $\mathrm{Sm}$ ) phase is destabilized either by polydispersity in the particle length or by curvature in the particle shape [65]. Inspired by these simulation results, a stable $N_{\mathrm{SB}}$ phase has indeed been experimentally observed, for the first time in a system of colloidal smoothly curved SU-8 rods [66] and in a system of polydisperse bent colloidal silica rods [67].

Despite these observations, many doubts subsist on the existence and the true nature of the $N_{\mathrm{SB}}$ phase. First, the splay-bend nematic phase has never been observed in systems of thermotropic bent-core mesogens, and only a huge variety of smectic phases has been found [49,164,165]. Moreover, the $N_{\mathrm{SB}}$ phases found in simulations of hard curved rods display density modulations, such that the nematic nature of the $N_{\mathrm{SB}}$ phase has been questioned. The presence of these density modulations in splay-bend phases has also been predicted by a Maier-Saupe theory [166].

In this Chapter we investigate the nature of the $N_{\mathrm{SB}}$ phase displayed in colloidal systems, by employing the Landau-de Gennes ( $\mathrm{LdG}$ ) theory that we have introduced in Chapter 4 of this thesis for lyotropic curved rods. We re-examine the theoretical predictions of Meyer [50] and Dozov [51] and show that the predicted $N-N_{\mathrm{SB}}$ transition is strictly speaking an $N-\mathrm{Sm}$ transition. In fact, we show that the splay deformations in the $N_{\mathrm{SB}}$ phase are accompanied by periodic density modulations, and hence the splay-bend phase exhibits positional order next to orientational ordering, in agreement with the simulation findings of Refs. $[65,166]$.

Meyer and Dozov explained the origin of bend deformations, and the concomitant onset of spatially modulated director fields in bent-core liquid crystals, on the basis of two different extensions of Oseen-Frank type theories for the nematic director field $\hat{\mathbf{n}}$. Meyer invoked that bend deformations originate from the spontaneous polar ordering of the particles which is, according to this framework, coupled to bend deformations in the nematic director field $\hat{\mathbf{n}}$, through a mechanism called the bend flexoelectric effect [82]. By contrast, Dozov did not consider any polar order and explained the bend distortions from purely elastic considerations, by speculating that the bend elastic constant $K_{33}$ in the Oseen-Frank energy of certain bentcore liquid crystals could become negative. Bend deformations would then arise spontaneously from the competition between the negative $K_{33}$ term and the positive higher-order elastic terms in the Oseen-Frank free energy. Recently Selinger [53] pursued Meyer's idea further and introduced a Landau theory that combines the Oseen-Frank free energy for the nematic director $\hat{\mathbf{n}}$, the polar order $\mathbf{P}$ perpendicular to $\hat{\mathbf{n}}$, and the coupling between polar order and 
bend deformations. Very interestingly he suggested that the presence of polar order could provide the simplest explanation for the negative $K_{33}$ proposed by Dozov. By minimizing the free energy with respect to the polar order, Selinger obtained Dozov's effective free energy in terms of only the nematic director field $\hat{\mathbf{n}}$ with renormalized elastic constants. In this picture, $K_{33}$ remains always positive, while its renormalized version $K_{33}^{\text {eff }}$ decreases in magnitude and vanishes at a critical point where the uniaxial $N$ phase becomes unstable with respect to either an $N_{\mathrm{TB}}$ or an $N_{\mathrm{SB}}$ phase. Quantitatively, in agreement with Dozov, Selinger's model predicts a second order $N-N_{\text {TB }}$ phase transition if $K_{11}>2 K_{22}$, or a second order $N-N_{\mathrm{SB}}$ phase transition if $K_{11}<2 K_{22}$, with $K_{11}$ and $K_{22}$ the splay and twist elastic constants, respectively.

Interestingly, none of the theoretical treatments so far involves the scalar nematic order parameter $S$ and the density $\rho$. In this Chapter we employ the $\mathrm{LdG}$ theory introduced in Chapter 4 of this thesis for lyotropic liquid crystals, that does involve a coupling between $S$ and $\rho$. We show that the $N_{\mathrm{SB}}$ phase has a periodic spatially modulating $S(z)$. By contrast with existing LdG theories for thermotropics $[54,135,138,167]$, our theory provides a natural coupling between orientational and positional order. The spatially modulated scalar order parameter $S$ in the $N_{\mathrm{SB}}$ phase thus yields a one-dimensional density modulation, such that the $N_{\mathrm{SB}}$ phase has actually the key characteristics of a smectic rather than a nematic phase. This is not the case, however, for the $N_{\text {TB }}$ phase, for which we find that $S$ does not vary in space, such that the $N_{\mathrm{TB}}$ phase is a true nematic phase.

This Chapter is organized as follows: in Section 5.2 we quickly review our theory. In particular we show how an extended version of the model used in Chapter 4 is able to detect the presence of density modulations. In Section 5.3 we present the results of our analysis. Finally, in Section 5.4 we present our conclusions and a discussion.

\subsection{Theory}

We extend Selinger's theory [53] to a mesoscopic LdG theory where the director $\hat{\mathbf{n}}$ is replaced by the standard second-rank, symmetric, and traceless tensor $\mathbf{Q}(\mathbf{r})$ with Cartesian components $Q_{\alpha \beta}(\mathbf{r})$ for $\alpha, \beta=x, y, z$. The eigenvector of $\mathbf{Q}$, corresponding to the maximum modulus of a non-degenerate eigenvalue, defines the nematic director $\hat{\mathbf{n}}$ of the system [76]. A vector field $\mathbf{P}(\mathbf{r})$ with Cartesian coordinates $P_{\gamma}(\mathbf{r})$ for $\gamma=x, y, z$ describes the polar order in a direction perpendicular to $\hat{\mathbf{n}}$. In order to describe lyotropic systems that become ordered with increasing density, we follow Ref. [80] and set up a Landau expansion for which we will use the chemicalpotential dependent grand potential $\Omega(\mu)$ rather than the temperature dependent Helmholtz (or Gibbs) free energy $F(T)$. More specifically, for a system of hard bent rods modeled as curved or kinked rods of contour length $L$ and diameter $D$, at chemical potential $\mu$ in a macroscopic volume $V$, and at fixed temperature $T$, we write the excess-over-isotropic LdG grand potential as $\Delta \Omega(\mathbf{Q}, \mathbf{P})=\int_{V} d \mathbf{r} \Delta \omega$. The excess grand potential density $\Delta \omega$ is given by

$$
\begin{aligned}
\beta B_{2} \Delta \omega= & \frac{2}{3} a \beta\left(\mu^{*}-\mu\right) Q_{\alpha \beta} Q_{\beta \alpha}-\frac{4}{3} b Q_{\alpha \beta} Q_{\beta \lambda} Q_{\lambda \alpha}+\frac{4}{9} d Q_{\alpha \beta} Q_{\beta \alpha} Q_{\lambda \rho} Q_{\rho \lambda}+\frac{2}{9} l_{1}\left(\partial_{\alpha} Q_{\beta \lambda}\right)\left(\partial_{\alpha} Q_{\beta \lambda}\right) \\
& +\frac{2}{9} l_{2}\left(\partial_{\alpha} Q_{\alpha \lambda}\right)\left(\partial_{\beta} Q_{\beta \lambda}\right)+e_{2} P_{\alpha}\left(\delta_{\alpha \beta}+\frac{2}{S_{0}} Q_{\alpha \beta}\right) P_{\beta}+e_{4} P_{\alpha} P_{\alpha} P_{\beta} P_{\beta}-\lambda P_{\alpha}\left(\partial_{\beta} Q_{\alpha \beta}\right) \\
& +\kappa\left(\partial_{\alpha} P_{\beta}\right)\left(\partial_{\alpha} P_{\beta}\right),
\end{aligned}
$$

where, throughout the Chapter, we use Einstein's summation convention for repeated indices, and where $a, b, d, l_{1}, l_{2}, S_{0}, e_{4}, \lambda$ and $\kappa$ are phenomenological parameters. Observe that only the 
quadratic term $Q_{\alpha \beta} Q_{\beta \alpha}$ has a $\mu$-dependent prefactor that changes sign at the nematic spinodal $\mu^{*}$ and drives the phase transitions. We express $\Delta \omega$ in units of $\beta^{-1}=k_{B} T$ with $k_{B}$ the Boltzmann constant, and in units of $B_{2}=\pi L^{2} D / 4$ the second virial coefficient of long needles in the isotropic $(I)$ fluid phase, which is a convenient scaling volume for systems of rodlike particles. Stability of the grand potential expansion in the dilute limit requires $a>0$ and $e_{2}>0$, while stability with respect to an unlimited growth of $\mathbf{Q}, \mathbf{P}$ and $\partial_{\alpha} P_{\beta}$ requires that $d>0, e_{4}>0$, and $\kappa>0$, respectively. The sign of the $b$ term determines the nature of the $I-N$ transition, rodlike for $b>0$, platelike for $b<0$. The coefficients $2 / S_{0}$ and $\kappa$ represent the Q-P coupling and the flexoelectric $\mathbf{P}-\nabla \mathbf{Q}$ coupling, respectively. In order to describe a favored polarization perpendicular to the nematic director, leading to a bend flexoelectric effect, we set $S_{0}>0$. Finally the coefficients $l_{1}$ and $l_{2}$ are related to the Oseen-Frank elastic constants $[74,75]$ through the relations $\beta D K_{11}=\beta D K_{33}=4 S^{2}\left(2 l_{1}+l_{2}\right) /\left(\pi L^{2}\right)$ and $\beta D K_{22}=8 S^{2} l_{1} /\left(\pi L^{2}\right)$. As shown in Chapter 4, a complete mapping exists between our LdG theory and the Oseen-Frank theory of Selinger [53].

Minimization of the grand potential (5.1) with respect to $\mathbf{Q}$ at $\mathbf{P} \equiv 0, \nabla \mathbf{Q} \equiv \mathbf{0}$, and $\nabla \mathbf{P} \equiv \mathbf{0}$, predicts a first order $I-N$ transition at $\beta \mu_{I N} \equiv \beta \mu^{*}-b^{2} /(4 a d)$. The nematic order parameter equals $S_{I}(\mu)=0$ for $\mu \leq \mu_{I N}$, and

$$
S_{N}(\mu)=\frac{3 b}{8 d}\left(1+\sqrt{1-\frac{32 a d \beta\left(\mu^{*}-\mu\right)}{9 b^{2}}}\right)
$$

for $\mu>\mu_{I N}$, with a jump at $\mu=\mu_{I N}$. In order to describe the "Onsager"-type $I-N$ phase transition of uniaxial hard needles [157] we follow Ref. [80] and set $a=1.436, b=5.851, d=$ 3.693, and $\beta \mu^{*}=6.855$. The $I-N$ transition occurs at $\beta \mu=5.241$. As shown in Chapter 4 , the uniaxial $N$ phase becomes unstable with respect to an $N_{\mathrm{TB}}$ or $N_{\mathrm{SB}}$ phase at a critical $\mu$ corresponding to $K_{33}^{\mathrm{eff}}=0$, where the system either stabilizes an $N_{\mathrm{TB}}$ phase, if $l_{1}$ and $l_{2}$ are such that $K_{11}>2 K_{22}$, or an $N_{\mathrm{SB}}$ phase, if $l_{1}$ and $l_{2}$ are such that $K_{11}<2 K_{22}$.

The $N_{\mathrm{SB}}$ phase is described by an achiral nematic director and polarization vector

$$
\begin{aligned}
\hat{\mathbf{n}}_{\mathrm{SB}}(z) & =(\sin \phi(z), 0, \cos \phi(z)), \\
\mathbf{P}_{\mathrm{SB}}(z) & =P \psi(z)\left(-\cos ^{2} \phi(z), 0, \frac{1}{2} \sin 2 \phi(z)\right),
\end{aligned}
$$

where $\psi(z)=\cos (q z)$ and $\phi(z)=\theta \sin (q z)$, with $P, q$ and $\theta$ variational parameters that characterize the polarization amplitude, the wave number and the tilt angle, respectively. The $N_{\mathrm{TB}}$ phase is described by a chiral nematic director and polarization vector

$$
\begin{aligned}
& \hat{\mathbf{n}}_{\mathrm{TB}}(z)=(\sin \theta \cos (q z), \sin \theta \sin (q z), \cos \theta), \\
& \mathbf{P}_{\mathrm{TB}}(z)=P(\sin (q z), \cos (q z), 0) .
\end{aligned}
$$

The $N-N_{\mathrm{TB}}$ as well as $N-N_{\mathrm{SB}}$ phase transitions proceed in both cases without any jump in $S(\mu)$. As a consequence, $S(\mu)$ coincides with the $S_{N}(\mu)$ of Eq. (5.2), in both cases.

To have a complete description of the phase behavior displayed by lyotropic systems, the particle density $\rho$ rather than the chemical potential $\mu$ is of interest. We employ the thermodynamic identity $\partial(\Omega / V) /\left.\partial \mu\right|_{V, T}=-\rho$ to perform this conversion. To this end, we introduce the grand potential density $\omega_{I}$ of the isotropic $I$ state and define $\omega \equiv \omega_{I}+\Delta \omega_{b}$, where the excess bulk grand potential $\Delta \omega_{b}$ is defined as

$$
\beta B_{2} \Delta \omega_{b}=\frac{2}{3} a \beta\left(\mu^{*}-\mu\right) Q_{\alpha \beta} Q_{\beta \alpha}-\frac{4}{3} b Q_{\alpha \beta} Q_{\beta \lambda} Q_{\lambda \alpha}+\frac{4}{9} d Q_{\alpha \beta} Q_{\beta \alpha} Q_{\lambda \rho} Q_{\rho \lambda} .
$$


From $\partial\left(B_{2} \omega\right) / \partial \mu=-c$, with $c \equiv B_{2} \rho$ the dimensionless particle concentration, we find

$$
c(\mu)=c_{I}(\mu)+a S^{2}(\mu) .
$$

The particle concentration of the $I$ phase, $c_{I}(\mu)=-\partial\left(B_{2} \omega_{I}\right) / \partial \mu$, can be calculated within Onsager theory, by using an isotropic distribution function, such that $\beta \mu\left(c_{I}\right)=\log \left(c_{I} / 4 \pi\right)+2 c_{I}$ [157]. By inverting this relation we obtain $c_{I}(\mu)$ straightforwardly. Together with Eq. (5.6), we can determine $c$, for every value of $\mu$ for which $S(\mu)$ is known.

We observe that Eq. (5.6) not only allows us to convert a dependence on the chemical potential in a dependence on the particle density, but also expresses a coupling between the scalar order parameter $S$ and the particle concentration $c$. In this Chapter we employ this coupling to obtain insight in the structure of the $N_{\mathrm{SB}}$ phase.

The nematic order parameter $S$ is not involved in the Oseen-Frank theories of Dozov [51] and Selinger [53], while it has been assumed spatially constant in LdG theories for thermotropics $[138,167]$. Analogously we have assumed $S$ spatially constant in Chapter 4 . Here we investigate the possibility of a spatially dependent scalar order parameter in the spatially modulated phases. As becomes clear from Eq. (5.6), analyzing the spatial dependence of $S$ has an important physical meaning since it can reveal the presence of spatial modulations in the particle concentration; a phase with a one dimensional density modulation is a smectic phase rather than a nematic one.

We consider here a $z$-dependent nematic order parameter $S(z)$ in the definition of the tensorial order paramter $\mathbf{Q}$, i.e.

$$
Q_{\alpha \beta}(z)=\frac{3}{2} S(z)\left(n_{\alpha}(z) n_{\beta}(z)-\frac{\delta_{\alpha \beta}}{3}\right),
$$

as well as a spatial modulation $P(z)$ of the amplitude of the polar vectors $\mathbf{P}_{\mathrm{SB}}$ and $\mathbf{P}_{\mathrm{TB}}$ in Eqs. (5.3) and (5.4). We perform a full minimization of the grand potential (5.1), by first looking for the equilibrium wave number and tilt angle, i.e. we solve the equations $\partial \Delta \Omega / \partial q=0$ and $\partial \Delta \Omega / \partial \theta=0$. We then insert the obtained $q(\mu)$ and $\theta(\mu)$ back into the grand potential $\Delta \Omega$, and solve, at fixed chemical potential $\mu$, the system of Euler-Lagrange equations

$$
\begin{aligned}
\frac{\delta \Delta \Omega}{\delta S(z)} & =\partial_{z} \frac{\delta \Delta \Omega}{\delta\left(\partial_{z} S(z)\right)}, \\
\frac{\delta \Delta \Omega}{\delta P(z)} & =\partial_{z} \frac{\delta \Delta \Omega}{\delta\left(\partial_{z} P(z)\right)} .
\end{aligned}
$$

For the $N_{\mathrm{TB}}$ phase, we denote the extremal solutions by $q_{\mathrm{TB}}(\mu), \theta_{\mathrm{TB}}(\mu), S_{\mathrm{TB}}(\mu ; z)$ and $P_{\mathrm{TB}}(\mu ; z)$. For the $N_{\mathrm{SB}}$ phase, we denote the extremal solutions by $q_{\mathrm{SB}}(\mu), \theta_{\mathrm{SB}}(\mu), S_{\mathrm{SB}}(\mu ; z)$ and $P_{\mathrm{SB}}(\mu ; z)$.

\subsection{Results}

We first consider the coefficients $l_{1}=0.165 L^{2}, l_{2}=0.427 L^{2}, e_{2}=1, S_{0}=0.85, e_{4}=0.1, \kappa=$ $0.00001 L^{2}$, and $\lambda=0.07 L$. It follows that $K_{11} / K_{22}=2.294$ and that $\Delta \Omega_{\mathrm{TB}}<\Delta \Omega_{\mathrm{SB}}$ for every $\mu$. Moreover $\Delta \Omega_{\mathrm{TB}}-\Delta \Omega_{N}$ changes sign at $\beta \mu=5.363 \equiv \beta \mu_{N N_{\mathrm{TB}}}$ where an $N-N_{\mathrm{TB}}$ phase transition takes place. We plot $S_{\mathrm{TB}}$ and $P_{\mathrm{TB}}$ as a function of $z$ for several values of the chemical potential $\mu \equiv \mu_{N N_{\mathrm{TB}}}+\Delta \mu$, in Figs. 5.1(a) and 5.1(c), respectively. We consider $z$ to vary in the range $\in\left[0,2 \pi / q_{\mathrm{TB}}\right]$. We clearly find from Figs. 5.1(a) and 5.1(c) that $S_{\mathrm{TB}}$ and $P_{\mathrm{TB}}$ are 

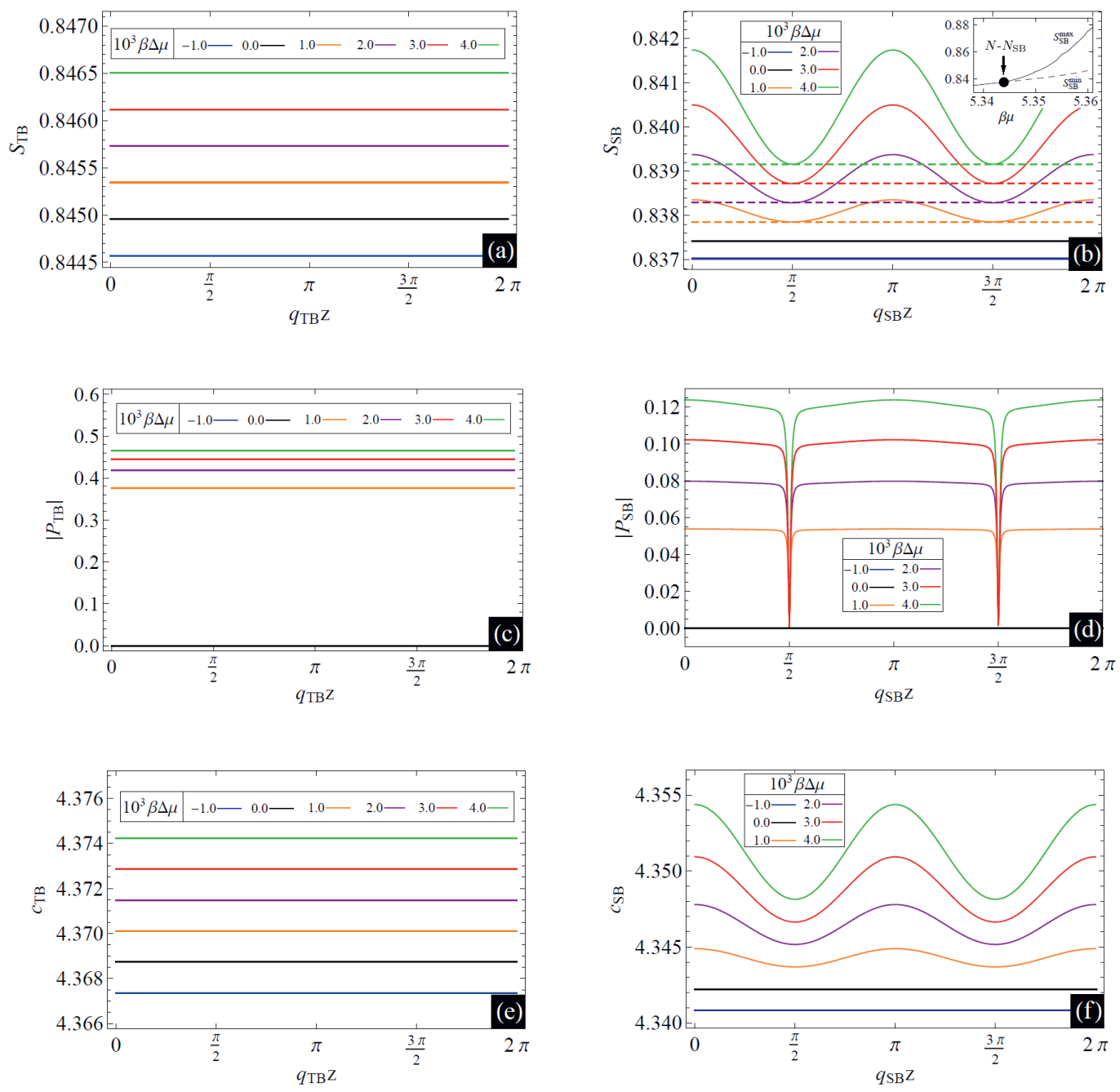

Figure 5.1: Nematic order parameter $S_{\mathrm{TB}}(a)$, modulus of the polarity $P_{\mathrm{TB}}(c)$, and particle concentration $c_{\mathrm{TB}}(e)$, in the $N_{\mathrm{TB}}$ phase, as a function of $z$, for several values of $\Delta \mu \equiv \mu-\mu_{N N_{\mathrm{TB}}}$. Nematic order parameter $S_{\mathrm{SB}}(b)$, modulus of the polarity $P_{\mathrm{SB}}(d)$, and particle concentration $c_{\mathrm{SB}}$ $(f)$, in the $N_{\mathrm{SB}}$ phase, as a function of $z$, for several values of $\Delta \mu \equiv \mu-\mu_{N N_{\mathrm{SB}}}$. While in $(a),(c)$ and $(e)$ we choose $l_{2}=0.427 L^{2}$, in $(b),(d)$ and $(f)$ we choose $l_{2}=0.0427 L^{2}$. The other coefficients are $l_{1}=0.165 L^{2}, e_{2}=1, S_{0}=0.85, e_{4}=0.1, \kappa=0.00001 L^{2}$ and $\lambda=0.07 L$, in all the cases. We observe that $S_{\mathrm{TB}}, P_{\mathrm{TB}}$, and $c_{\mathrm{TB}}$ remain constant along $z$ for each $\mu$. By contrast, we observe that $S_{\mathrm{SB}}, P_{\mathrm{SB}}$, and $c_{\mathrm{SB}}$ are constant along $z$ for $\mu \leq \mu_{N N_{\mathrm{SB}}}$, while they display modulations along $z$ for $\mu>\mu_{N N_{\mathrm{SB}}}$. 

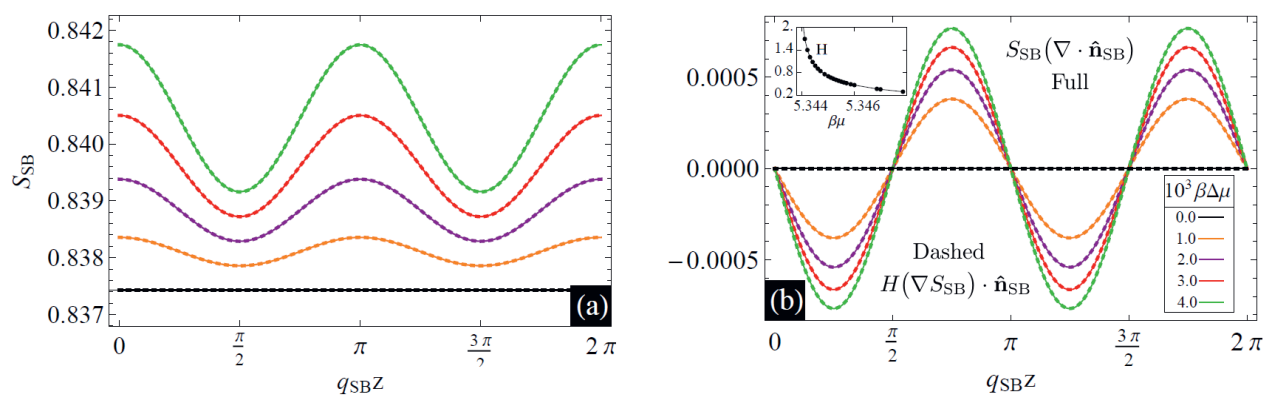

Figure 5.2: (a) With points and lines we plot the same $S_{\mathrm{SB}}(z)$ profiles depicted in Fig. 5.1(b), and a fit of the numerical results, respectively. The fitting function corresponds to Eq. (5.9) with coefficients given in Table 5.1. (b) With full and dashed lines we plot $S_{\mathrm{SB}}\left(\nabla \cdot \hat{\mathbf{n}}_{\mathrm{SB}}\right)$ and $H\left(\nabla S_{\mathrm{SB}}\right) \cdot \hat{\mathbf{n}}_{\mathrm{SB}}$, respectively, as a function of $z$, for the same values of the coefficients and of the chemical potential as in Figs. $5.1(b), 5.1(d)$ and $5.1(f) . H$ is a constant that varies with $\mu$, as showed in the inset of $(b)$. At each $\mu$, the relation $S_{\mathrm{SB}}\left(\nabla \cdot \hat{\mathbf{n}}_{\mathrm{SB}}\right)=H\left(\nabla S_{\mathrm{SB}}\right) \cdot \hat{\mathbf{n}}_{\mathrm{SB}}$ holds.

constant along $z$, for every $\mu$. In particular we find that $S_{\mathrm{TB}}(\mu)$ coincides with the $S_{N}(\mu)$ of Eq. (5.2). We then convert the $S_{\mathrm{TB}}(z)$ profile depicted in Fig. $5.1(a)$ into the $c_{\mathrm{TB}}(z)$ profile depicted in Fig. 5.1(e). As can be clearly observed, $c_{\mathrm{TB}}$ is constant along $z$. We conclude, as expected, that spatial modulations in twist and bend do not affect the nematic ordering.

Subsequently, we set $l_{2}=0.0427 L^{2}$. It follows that $K_{11} / K_{22}=1.129$ and that $\Delta \Omega_{\mathrm{SB}}<$ $\Delta \Omega_{\mathrm{TB}}$ for every $\mu$. Moreover $\Delta \Omega_{\mathrm{SB}}-\Delta \Omega_{N}$ changes sign at $\beta \mu=5.34386 \equiv \beta \mu_{N N_{\mathrm{SB}}}$, where an $N-N_{\mathrm{SB}}$ phase transition takes place. We plot $S_{\mathrm{SB}}$ and $P_{\mathrm{SB}}$ as a function of $z$ for several values of the chemical potential $\mu \equiv \mu_{N N_{\mathrm{SB}}}+\Delta \mu$, in Figs. 5.1(b) and 5.1(d), respectively. We consider $z$ to vary in the range $\in\left[0,2 \pi / q_{\mathrm{SB}}\right]$. By contrast with the case of the $N_{\mathrm{TB}}$ phase, we find that, while for $\mu \leq \mu_{N N_{\mathrm{SB}}} S_{\mathrm{SB}}$ and $P_{\mathrm{SB}}$ are constant along $z$, for $\mu>\mu_{N N_{\mathrm{SB}}}$ they start to display modulations. In particular we find that, close to the $N-N_{\mathrm{SB}}$ transition point, $S_{N}(\mu)$ coincides with the minimum of the $S_{\mathrm{SB}}(\mu)$ profile. In the inset of Fig. $5.1(b)$ we plot the maximum $S_{\mathrm{SB}}^{\max }$ and the minimum $S_{\mathrm{SB}}^{\min }$ of the $S_{\mathrm{SB}}$ profile, as a function of $\mu$. We find that the amplitude of $S_{\mathrm{SB}}$ increases with the chemical potential. Finally, we convert the $S_{\mathrm{SB}}(z)$ profile depicted in Fig. $5.1(b)$ into the $c_{\mathrm{SB}}(z)$ profile depicted in Fig. $5.1(f)$. In analogy with $S_{\mathrm{SB}}$ and $P_{\mathrm{SB}}$ we observe that, also $c_{\mathrm{SB}}$ displays modulations along $z$, for $\mu>\mu_{N N_{\mathrm{SB}}}$. It follows that the $N_{\mathrm{SB}}$ phase has all the characteristics of a smectic (or lamellar) phase.

Table 5.1: Coefficients $\theta_{S}$ of the functional form (5.9), as a function of $\mu$, obtained through fitting the numerical results.

\begin{tabular}{ll}
\hline $10^{3} \beta \Delta \mu$ & $\theta_{S}$ \\
\hline 0.0 & 0.0 \\
1.0 & 0.0345137 \\
2.0 & 0.0510039 \\
3.0 & 0.0651242 \\
4.0 & 0.0784338 \\
\hline
\end{tabular}

We now argue that the density modulations depicted in Fig. 5.1 $(f)$ are coupled with the splay deformations of the nematic director field $\hat{\mathbf{n}}_{\mathrm{SB}}$. To this aim, we first note (see Fig. 5.2(a)) 

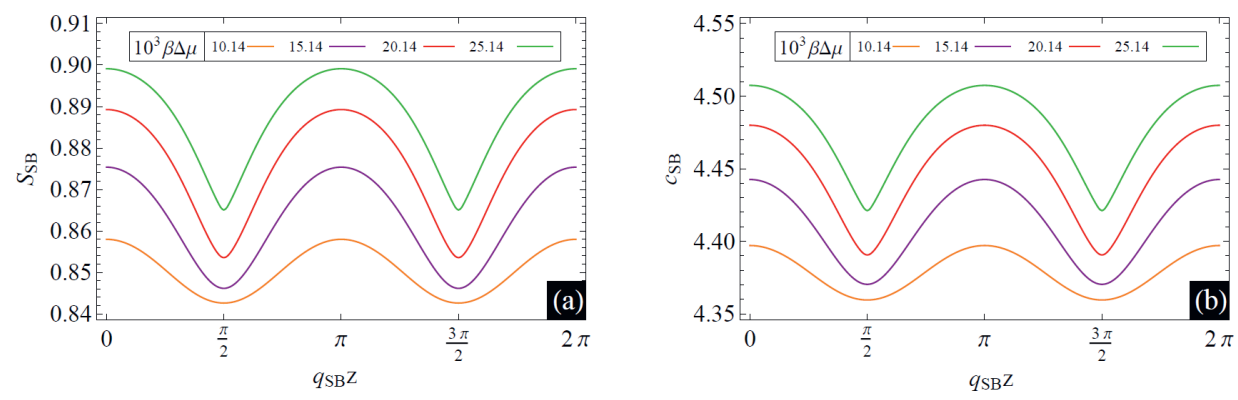

Figure 5.3: Same as Figs. 5.1(b) and 5.1(f), but now for larger values of $\Delta \mu \equiv \mu-\mu_{N N_{\mathrm{SB}}}$.

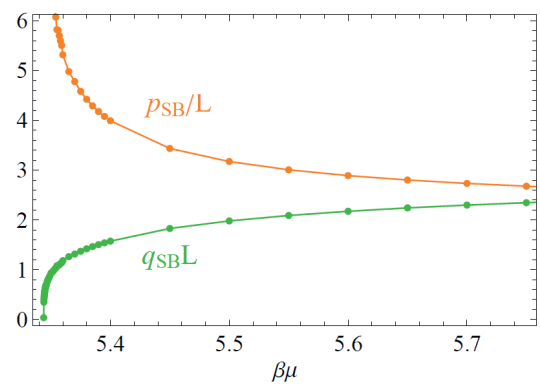

Figure 5.4: Equilibrium wave number $q_{\mathrm{SB}}$ and equilibrium pitch $p_{\mathrm{SB}} \equiv 2 \pi / q_{\mathrm{SB}}$ in the $N_{\mathrm{SB}}$ phase, as a function of the chemical potential $\mu$, for the same coefficients as in Figs. 5.1(b), 5.1(d) and 5.1(f).

that the $S_{\mathrm{SB}}(z)$ profiles numerically obtained in Fig. 5.1(b) fit very well with the functional form

$$
S_{\mathrm{SB}}(z)=S_{\mathrm{SB}}^{\max } \cos \left(\theta_{S} \sin q_{\mathrm{SB}} z\right),
$$

where, for several $\Delta \mu, \theta_{S}$ is reported in Table 5.1. Then, for the same values of $\mu$ considered in Fig. $5.1(b)$, we plot in Fig. $5.2(b), S_{\mathrm{SB}}\left(\nabla \cdot \hat{\mathbf{n}}_{\mathrm{SB}}\right)$ and $H\left(\nabla S_{\mathrm{SB}}\right) \cdot \hat{\mathbf{n}}_{\mathrm{SB}}$ as a function of $z$, with full and dashed lines, respectively. We observe that $H$ is a spatial constant that varies with $\mu$, as shown in the inset of Fig. 5.2(b). We observe that for each $\mu$, the condition $S_{\mathrm{SB}}\left(\nabla \cdot \hat{\mathbf{n}}_{\mathrm{SB}}\right)=H\left(\nabla S_{\mathrm{SB}}\right) \cdot \hat{\mathbf{n}}_{\mathrm{SB}}$ holds, from which we can derive (see Appendix A)

$$
\nabla \cdot \hat{\mathbf{n}}_{\mathrm{SB}}(z)=\frac{H}{2} \frac{\nabla c_{\mathrm{SB}}(z)}{\left(c_{\mathrm{SB}}(z)-c_{I}\right)} \cdot \hat{\mathbf{n}}_{\mathrm{SB}}(z)
$$

through Eq. (5.6). We observe that an expression similar to the one of Eq. (5.10) was found by de Gennes [168] and Taratuta and Meyer [169] for polymer nematics. It expresses the coupling between concentration gradients and splay deformations, given by $\nabla \cdot \hat{\mathbf{n}}_{\mathrm{SB}}$, which implies that there can not be splay deformation without a change in local density.

Finally, we find that the shape of $S_{\mathrm{SB}}(z)$ and $c_{\mathrm{SB}}(z)$ depicted in Figs. $5.1(b)$ and $5.1(f)$, changes by considering higher values of the chemical potential $\mu$. To show this, we plot $S_{\mathrm{SB}}(z)$ and $c_{\mathrm{SB}}(z)$ for several values of $\mu \gg \mu_{N N_{\mathrm{SB}}}$ in Figs. 5.3(a) and 5.3(b), respectively. We observe that the density modulations displayed by the $N_{\mathrm{SB}}$ phase along $z$ in the regime $\mu \rightarrow \mu_{N N_{\mathrm{SB}}}^{+}$is different from the one in the regime $\mu \gg \mu_{N N_{\mathrm{SB}}}$. In particular, we find that in the latter regime 
$S_{\mathrm{SB}}$ does not fit with the functional form (5.9), and that the relation (5.10) does not hold anymore. We also observe that in the two regimes, the $N_{\mathrm{SB}}$ is characterized by different values of the equilibrium pitch $p_{\mathrm{SB}} \equiv 2 \pi / q_{\mathrm{SB}}$. As shown in Fig. 5.4 , while $p_{\mathrm{SB}} \gg L$ for $\mu \rightarrow \mu_{N N_{\mathrm{SB}}}^{+}$, $p_{\mathrm{SB}} \sim L$ for $\mu \gg \mu_{N N_{\mathrm{SB}}}$.

\subsection{Conclusions and discussion}

In this Chapter we have employed the LdG theory introduced in Chapter 4 of this thesis for lyotropic colloidal suspensions of bent rods, to investigate the effect of spatial distortions in the nematic director field on the density of the twist-bend and splay-bend nematic phases. In contrast with the existing Oseen-Frank theories [50,51,53], our theory allows us to analyze the spatial dependence of the nematic order parameter $S$, in addition to that of the nematic director field $\hat{\mathbf{n}}$. Moreover, by contrast with LdG theories for thermotropic liquid crystals [54, $135,138,167]$, our theory provides a natural coupling between $S$ and the particle density $\rho$. We show that the $N_{\mathrm{SB}}$ phase is characterized by a spatially modulated $S(z)$, and hence by a onedimensional density modulation $\rho(z)$. It follows that the originally predicted $N-N_{\mathrm{SB}}$ transition is strictly speaking a $N$-Sm transition. In the case of an $N_{\text {TB }}$ phase, we find that $S$ is constant in space such that this phase does not display any density modulation, and is a true nematic phase. Moreover we find that, close to the $N-N_{\mathrm{SB}}$ transition point, the density modulations are coupled with splay deformations in the nematic director field $\hat{\mathbf{n}}$.

In addition it is interesting to also investigate the $N_{\mathrm{TB}}-N_{\mathrm{SB}}$ phase transition within this framework. By using both simulations and a generalized Maier-Saupe mean-field theory [166], Chiappini and Dijkstra showed that, in systems of curved rods, an $N_{\mathrm{TB}}-N_{\mathrm{SB}}$ phase transition occurs continuously, via an intermediate elliptical analogue of the $N_{\mathrm{TB}}$ phase which they have named twist-splay-bend $\left(N_{\mathrm{TSB}}\right)$ phase. In analogy with the $N_{\mathrm{SB}}$ phase, they have shown that the $N_{\text {TSB }}$ phase displays density modulations, such that the appearance of density modulations would be inherently associated with splay deformations in the nematic director field $\hat{\mathbf{n}}$. As shown in Chapter 4 of this thesis, an $N_{\mathrm{TB}}-N_{\mathrm{SB}}$ transition is described by our LdG theory if the degeneracy between the $K_{11}$ and $K_{33}$ elastic constants is removed by adding a term that involves the coupling between the order parameter $\mathbf{Q}$ and quadratic gradient in $\mathbf{Q}$, to the expansion (5.1). The LdG theory and the minimization procedure presented in this Chapter can be employed to verify the existence and the true nature of the putative $N_{\text {TSB }}$ phase. We plan to address these issues in future work.

\subsection{Appendix A: Derivation of Eq. (5.10)}

As shown in Fig. 5.2(b) a spatial constant $H$ can be found, at each $\mu$, such that

$$
S_{\mathrm{SB}}\left(\nabla \cdot \hat{\mathbf{n}}_{\mathrm{SB}}\right)=H\left(\nabla S_{\mathrm{SB}}\right) \cdot \hat{\mathbf{n}}_{\mathrm{SB}} \cdot
$$

By using the thermodynamic identity (5.6), we can write 


$$
\begin{aligned}
\frac{\nabla S_{\mathrm{SB}}}{S_{\mathrm{SB}}} & =\frac{2 S_{\mathrm{SB}}\left(\nabla S_{\mathrm{SB}}\right)}{2 S_{\mathrm{SB}}^{2}} \\
& =\frac{\nabla S_{\mathrm{SB}}^{2}}{2 S_{\mathrm{SB}}^{2}} \\
& =\frac{\nabla\left(\frac{c_{\mathrm{SB}}(z)-c_{I}}{a}\right)}{2 \frac{c_{\mathrm{SB}}(z)-c_{I}}{a}} \\
& =\frac{\nabla c(z)}{2\left(c_{\mathrm{SB}}(z)-c_{I}\right)} .
\end{aligned}
$$

Inserting (5.12) into (5.11) we find Eq. (5.10). 


\section{References}

[1] P. G. de Gennes, Soft matter, Rev. Mod. Phys. 64, 645 (1992).

[2] R. Brown, A brief account of microscopical observations made in the months of june, july and august 1827, on the particles contained in the pollen of plants; and on the general existence of active molecules in organic and inorganic bodies, The Philosophical Magazine 4, 161 (1828).

[3] A. Einstein, Über die von der molekularkinetischen theorie der wärme geforderte bewegung von in ruhenden flü̈sigkeiten suspendierten teilchen, Ann. Phys. (Leipzig) 17, 549 (1905).

[4] J. Perrin, Mouvement brownien et réalité moléculaire, Ann. Chim. Phys. 18, 5 (1909).

[5] G. L. Hunter and E. R. Weeks, The physics of the colloidal glass transition, Reports on Progress in Physics 75, 066501 (2012).

[6] F. Sciortino and P. Tartaglia, Glassy colloidal systems, Advances in Physics 54, 471 (2005).

[7] A. Yethiraj and A. van Blaaderen, A colloidal model system with an interaction tunable from hard sphere to soft and dipolar, Nature 421, 513 (2003).

[8] S. C. Glotzer and M. J. Solomon, Anisotropy of building blocks and their assembly into complex structures, Nature Materials 6, 557 (2007).

[9] A. van Blaaderen and P. Wiltzius, Real-space structure of colloidal hard-sphere glasses, Science 270, 1177 (1995).

[10] W. Ramsden, Separation of Solids in the Surface-Layers of Solutions and "Suspensions" ( Observations on Surface-Membranes, Bubbles, Emulsions, and Mechanical Coagulation ). , Proceedings of the Royal Society of London 72, 156 (1903).

[11] S. U. Pickering, Emulsions, J. Chem. Soc. 91, 2001 (1907).

[12] P. Pieranski, Two-Dimensional Interfacial Colloidal Crystals, Phys. Rev. Lett. 45, 569 (1980).

[13] P. G. de Gennes, Wetting: statics and dynamics, Rev. Mod. Phys. 57, 827 (1985).

[14] P. A. Kralchevsky and K. Nagayama, Capillary interactions between particles bound to interfaces, liquid films and biomembranes, Advances in Colloid and Interface Science 85, 145 (2000).

[15] R. McGorty, J. Fung, D. Kaz, and V. N. Manoharan, Colloidal self-assembly at an interface, Materials Today 13, 34 (2010).

[16] S. Sacanna, W. K. Kegel, and A. P. Philipse, Thermodynamically stable pickering emulsions, Physical Review Letters 98, 13 (2007).

[17] K. Stratford, R. Adhikari, I. Pagonabarraga, J.-C. Desplat, and M. E. Cates, Colloidal Jamming at Interfaces: A Route to Fluid-Bicontinuous Gels, Science 309, 2198 (2005). 
[18] S. Sacanna and D. J. Pine, Shape-anisotropic colloids: Building blocks for complex assemblies, Current Opinion in Colloid \& Interface Science 16, 96 (2011).

[19] E. P. Lewandowski, J. A. Bernate, A. Tseng, P. C. Searson, and K. J. Stebe, Oriented assembly of anisotropic particles by capillary interactions, Soft Matter 5, 886 (2009).

[20] E. P. Lewandowski, M. Cavallaro, L. Botto, J. C. Bernate, V. Garbin, and K. J. Stebe, Orientation and Self-Assembly of Cylindrical Particles by Anisotropic Capillary Interactions, Langmuir 26, $15142(2010)$.

[21] L. Botto, E. P. Lewandowski, M. Cavallaro, and K. J. Stebe, Capillary interactions between anisotropic particles, Soft Matter 8, 9957 (2012).

[22] A. B. Liu, N. Sharifi-Mood, and K. J. Stebe, Capillary Assembly of Colloids: Interactions on Planar and Curved Interfaces, Annual Review of Condensed Matter Physics 9, 283 (2018).

[23] B. Madivala, J. Fransaer, and J. Vermant, Self-Assembly and Rheology of Ellipsoidal Particles at Interfaces, Langmuir 25, 2718 (2009).

[24] S. Dasgupta, M. Katava, M. Faraj, T. Auth, and G. Gompper, Capillary Assembly of Microscale Ellipsoidal, Cuboidal, and Spherical Particles at Interfaces, Langmuir 30, 11873 (2014).

[25] S. Dasgupta, T. Auth, and G. Gompper, Nano- and microparticles at fluid and biological interfaces, Journal of Physics: Condensed Matter 29, 373003 (2017).

[26] B. J. Park and D. Lee, Configuration of nonspherical amphiphilic particles at a fluid-fluid interface, Soft Matter 8, 7690 (2012).

[27] B. J. Park and D. Lee, Equilibrium Orientation of Nonspherical Janus Particles at Fluid-Fluid Interfaces, ACS Nano 6, 782 (2012).

[28] L. C. Bradley, W.-H. Chen, K. J. Stebe, and D. Lee, Janus and patchy colloids at fluid interfaces, Current Opinion in Colloid \& Interface Science 30, 25 (2017).

[29] F. Chang, Patchy colloids with orthogonal functionality, PhD thesis, Utrecht University, 2019.

[30] C. Anzivino, F. Chang, G. Soligno, R. van Roij, W. K. Kegel, and M. Dijkstra, Equilibrium configurations and capillary interactions of Janus dumbbells and spherocylinders at fluid-fluid interfaces, Soft Matter 15, 2638 (2019).

[31] Q. Song, M. Steuber, S. I. Druzhinin, and H. Schönherr, Tailored Combinatorial Microcompartments through the Self-Organization of Microobjects: Assembly, Characterization, and Cell Studies, Angewandte Chemie International Edition 58, 5246 (2019).

[32] Q. Song and H. Schönherr, Control of Orientation, Formation of Ordered Structures, and SelfSorting of Surface-Functionalized Microcubes at the Air-Water Interface, Langmuir 35, 6742 (2019).

[33] G. Soligno, M. Dijkstra, and R. van Roij, Self-Assembly of Cubes into 2D Hexagonal and Honeycomb Lattices by Hexapolar Capillary Interactions, Phys. Rev. Lett. 116, 258001 (2016).

[34] G. Soligno, M. Dijkstra, and R. van Roij, Self-assembly of cubic colloidal particles at fluid-fluid interfaces by hexapolar capillary interactions, Soft Matter 14, 42 (2018).

[35] F. C. Bawden, N. W. Pirie, J. D. Bernal, and I. Fankuchen, Liquid Crystalline Substances from Virus-infected Plants, Nature 138, 1051 (1936). 
[36] L. Onsager, The effects of shape on the interaction of colloidal particles, Annals of the New York Academy of Sciences 51, 627 (1949).

[37] D. Frenkel, H. N. W. Lekkerkerker, and A. Stroobants, Thermodynamic stability of a smectic phase in a system of hard rods, Nature 332, 822 (1988).

[38] A. Stroobants, H. N. W. Lekkerkerker, and D. Frenkel, Evidence for smectic order in a fluid of hard parallel spherocylinders, Phys. Rev. Lett. 57, 1452 (1986).

[39] Z. Dogic and S. Fraden, Smectic phase in a colloidal suspension of semiflexible virus particles, Phys. Rev. Lett. 78, 2417 (1997).

[40] D. Frenkel, Columnar ordering as an excluded-volume effect, Liquid Crystals 5, 929 (1989).

[41] J. A. C. Veerman and D. Frenkel, Relative stability of columnar and crystalline phases in a system of parallel hard spherocylinders, Phys. Rev. A 43, 4334 (1991).

[42] S. Belli, S. Dussi, M. Dijkstra, and R. van Roij, Density functional theory for chiral nematic liquid crystals, Phys. Rev. E 90, 020503 (2014).

[43] S. Dussi, S. Belli, R. van Roij, and M. Dijkstra, Cholesterics of colloidal helices: Predicting the macroscopic pitch from the particle shape and thermodynamic state, The Journal of Chemical Physics 142, 074905 (2015).

[44] E. Grelet and S. Fraden, What is the origin of chirality in the cholesteric phase of virus suspensions?, Phys. Rev. Lett. 90, 198302 (2003).

[45] M. J. Freiser, Ordered states of a nematic liquid, Phys. Rev. Lett. 24, 1041 (1970).

[46] C. Tschierske and D. J. Photinos, Biaxial nematic phases, J. Mater. Chem. 20, 4263 (2010).

[47] S. Belli, A. Patti, M. Dijkstra, and R. van Roij, Polydispersity stabilizes biaxial nematic liquid crystals, Phys. Rev. Lett. 107, 148303 (2011).

[48] P. I. C. Teixeira, A. J. Masters, and B. M. Mulder, Biaxial nematic order in the hard-boomerang fluid, Molecular Crystals and Liquid Crystals Science and Technology. Section A. Molecular Crystals and Liquid Crystals 323, 167 (1998).

[49] A. Jákli, O. D. Lavrentovich, and J. V. Selinger, Physics of liquid crystals of bent-shaped molecules, Rev. Mod. Phys. 90, 45004 (2018).

[50] R. B. Meyer, Structural problems in liquid crystal physics, in Molecular Fluids, edited by R. Balian and G. Weill, Les Houches Summer School in Theoretical Physics, pages 271-343, Gordon and Breach, New York, 1976.

[51] I. Dozov, On the spontaneous symmetry breaking in the mesophases of achiral banana-shaped molecules, Europhysics Letters 56, 247 (2001).

[52] S. Dhakal and J. V. Selinger, Statistical mechanics of splay flexoelectricity in nematic liquid crystals, Phys. Rev. E 81, 31704 (2010).

[53] S. M. Shamid, S. Dhakal, and J. V. Selinger, Statistical mechanics of bend flexoelectricity and the twist-bend phase in bent-core liquid crystals, Phys. Rev. E 87, 52503 (2013).

[54] S. M. Shamid, D. W. Allender, and J. V. Selinger, Predicting a polar analog of chiral blue phases in liquid crystals, Phys. Rev. Lett. 113, 237801 (2014). 
[55] E. G. Virga, Double-well elastic theory for twist-bend nematic phases, Phys. Rev. E 89, 52502 (2014).

[56] G. Barbero, L. R. Evangelista, M. P. Rosseto, R. S. Zola, and I. Lelidis, Elastic continuum theory: towards understanding of the twist-bend nematic phases, Phys. Rev. E 92, 30501 (2015).

[57] R. S. Zola, G. Barbero, I. Lelidis, M. P. Rosseto, and L. R. Evangelista, A continuum description for cholesteric and nematic twist-bend phases based on symmetry considerations, Liquid Crystals 44, 24 (2017).

[58] I. Lelidis and G. Barbero, Nematic phases with spontaneous splay-bend deformation: standard elastic description, Liquid Crystals 43, 208 (2016).

[59] E. I. Kats and V. V. Lebedev, Landau theory for helical nematic phases, JETP Letters 100, 110 (2014).

[60] T. C. Lubensky and L. Radzihovsky, Theory of bent-core liquid-crystal phases and phase transitions, Phys. Rev. E 66, 31704 (2002).

[61] Y. Yang, H. Pei, G. Chen, K. T. Webb, L. J. Martinez-Miranda, I. K. Lloyd, Z. Lu, K. Liu, and Z. Nie, Phase behaviors of colloidal analogs of bent-core liquid crystals, Science Advances 4 (2018).

[62] Y. Yang, G. Chen, L. J. Martinez-Miranda, H. Yu, K. Liu, and Z. Nie, Synthesis and liquidcrystal behavior of bent colloidal silica rods, Journal of the American Chemical Society 138, 68 (2016).

[63] F. Hagemans, R. K. Pujala, D. S. Hotie, D. M. Thies-Weesie, D. A. De Winter, J. D. Meeldijk, A. Van Blaaderen, and A. Imhof, Shaping silica rods by tuning hydrolysis and condensation of silica precursors, Chemistry of Materials 31, 521 (2019).

[64] C. Greco and A. Ferrarini, Entropy-driven chiral order in a system of achiral bent particles, Phys. Rev. Lett. 115, 147801 (2015).

[65] M. Chiappini, T. Drwenski, R. van Roij, and M. Dijkstra, Biaxial, twist-bend, and splay-bend nematic phases of banana-shaped particles revealed by lifting the "smectic blanket", Phys. Rev. Lett. 123, 68001 (2019).

[66] C. Fernández-Rico, M. Chiappini, T. Yanagishima, H. de Sousa, D. G. A. L. Aarts, M. Dijkstra, and R. P. A. Dullens, Shaping colloidal bananas to reveal biaxial, splay-bend nematic, and smectic phases, Science 369, 950 (2020).

[67] R. Kotni, A. Grau-Carbonell, M. Chiappini, M. Dijkstra, and A. van Blaaderen, Splay-bend nematic phases of bent colloidal silica rods induced by polydispersity (unpublished).

[68] G. Soligno, M. Dijkstra, and R. van Roij, The equilibrium shape of fluid-fluid interfaces: Derivation and a new numerical method for Young's and Young-Laplace equations, The Journal of Chemical Physics 141, 244702 (2014).

[69] G. Soligno, Droplets, capillary interactions and self-assembly from the equilibrium shape of fluid-fluid interfaced, PhD thesis, Utrecht University, 2017.

[70] S. Kirkpatrick, C. D. Gelatt, and M. P. Vecchi, Optimization by simulated annealing, Science 220, 671 (1983). 
[71] R. Evans, The nature of the liquid-vapour interface and other topics in the statistical mechanics of non-uniform, classical fluids, Advances in Physics 28, 143 (1979).

[72] D. Frenkel and B. Smith, Understanding Molecular Simulations: From Algorithms to Applications, Academic Press, 2002.

[73] M. P. Allen and D. J. Tildesley, Computer Simulation of Liquids, Oxford University Press, 2017.

[74] C. W. Oseen, The theory of liquid crystals, Trans. Faraday Soc. 29, 883 (1933).

[75] F. C. Frank, I. Liquid crystals. On the theory of liquid crystals, Discuss. Faraday Soc. 25, 19 (1958).

[76] P.-G. de Gennes and J. Prost, The Physics of Liquid Crystals, Oxford University Press, 1993.

[77] S.-K. Ma, Statistical Mechanics, World Scientific, 1985.

[78] A. B. Harris, R. D. Kamien, and T. C. Lubensky, Molecular chirality and chiral parameters, Rev. Mod. Phys. 71, 1745 (1999).

[79] P. G. D. Gennes, Short range order effects in the isotropic phase of nematics and cholesterics, Molecular Crystals and Liquid Crystals 12, 193 (1971).

[80] J. C. Everts, M. T. J. J. M. Punter, S. Samin, P. van der Schoot, and R. van Roij, A Landau-de Gennes theory for hard colloidal rods: defects and tactoids, The Journal of Chemical Physics 144, 194901 (2016).

[81] K. Schiele and S. Trimper, On the elastic constants of a nematic liquid crystal, Physica Status Solidi (b) 118, 267 (1983).

[82] R. B. Meyer, Piezoelectric effects in liquid crystals, Phys. Rev. Lett. 22, 918 (1969).

[83] F. Bresme and M. Oettel, Nanoparticles at fluid interfaces, Journal of Physics: Condensed Matter 19, 413101 (2007).

[84] D. Vella and L. Mahadevan, The "Cheerios effect", American Journal of Physics 73, 817 (2005).

[85] D. Y. C. Chan, J. D. Henry, and L. R. White, The interaction of colloidal particles collected at fluid interfaces, Journal of Colloid and Interface Science 79, 410 (1981).

[86] E. P. Lewandowski, P. C. Searson, and K. J. Stebe, Orientation of a Nanocylinder at a Fluid Interface, The Journal of Physical Chemistry B 110, 4283 (2006).

[87] D. Ershov, J. Sprakel, J. Appel, M. A. Cohen Stuart, and J. van der Gucht, Capillarity-induced ordering of spherical colloids on an interface with anisotropic curvature, Proceedings of the National Academy of Sciences 110, 9220 (2013).

[88] R. Di Leonardo, F. Saglimbeni, and G. Ruocco, Very-Long-Range Nature of Capillary Interactions in Liquid Films, Phys. Rev. Lett. 100, 106103 (2008).

[89] J. N. Israelachvili, Intermolecular and surface forces, Academic Press London; San Diego, 1991.

[90] N. Bowden, A. Terfort, J. Carbeck, and G. M. Whitesides, Self-Assembly of Mesoscale Objects into Ordered Two-Dimensional Arrays, Science 276, 233 (1997).

[91] J. C. Loudet, A. M. Alsayed, J. Zhang, and A. G. Yodh, Capillary Interactions Between Anisotropic Colloidal Particles, Phys. Rev. Lett. 94, 18301 (2005). 
[92] D. Stamou, C. Duschl, and D. Johannsmann, Long-range attraction between colloidal spheres at the air-water interface: The consequence of an irregular meniscus, Phys. Rev. E 62, 5263 (2000).

[93] S. Coertjens, P. Moldenaers, J. Vermant, and L. Isa, Contact Angles of Microellipsoids at Fluid Interfaces, Langmuir 30, 4289 (2014).

[94] M. Zanini and L. Isa, Particle contact angles at fluid interfaces: pushing the boundary beyond hard uniform spherical colloids, Journal of Physics: Condensed Matter 28, 313002 (2016).

[95] G. Morris, K. Hadler, and J. Cilliers, Particles in thin liquid films and at interfaces, Current Opinion in Colloid \& Interface Science 20, 98 (2015).

[96] N. Sharifi-Mood, I. B. Liu, and K. J. Stebe, Capillary interactions on fluid interfaces: Opportunities for directed assembly, Soft Matter Self-Assembly 193, 165 (2016).

[97] T. G. Anjali and M. G. Basavaraj, Shape-Induced Deformation, Capillary Bridging, and SelfAssembly of Cuboids at the Fluid-Fluid Interface, Langmuir 33, 791 (2017).

[98] M. Cavallaro, L. Botto, E. P. Lewandowski, M. Wang, and K. J. Stebe, Curvature-driven capillary migration and assembly of rod-like particles, Proceedings of the National Academy of Sciences 108, 20923 (2011).

[99] C. Casagrande, P. Fabre, E. Raphaël, and M. Veyssié, "Janus Beads": Realization and behaviour at water/oil interfaces, Europhysics Letters, 251.

[100] A. Kumar, B. J. Park, F. Tu, and D. Lee, Amphiphilic Janus particles at fluid interfaces, Soft Matter 9, 6604 (2013).

[101] B. J. Park, C.-H. Choi, S.-M. Kang, K. E. Tettey, C.-S. Lee, and D. Lee, Double Hydrophilic Janus Cylinders at an Air-Water Interface, Langmuir 29, 1841 (2013).

[102] D. W. Kang, W. Ko, B. Lee, and B. J. Park, Effect of Geometric and Chemical Anisotropy of Janus Ellipsoids on Janus Boundary Mismatch at the Fluid-Fluid Interface, Materials 9 (2016).

[103] L. Isa, N. Samudrala, and E. R. Dufresne, Adsorption of Sub-Micron Amphiphilic Dumbbells to Fluid Interfaces, Langmuir 30, 5057 (2014).

[104] K.-H. Roh, D. C. Martin, and J. Lahann, Biphasic Janus particles with nanoscale anisotropy, Nature Materials 4, 759 (2005).

[105] J.-W. Kim, R. J. Larsen, and D. A. Weitz, Synthesis of Nonspherical Colloidal Particles with Anisotropic Properties, Journal of the American Chemical Society 128, 14374 (2006).

[106] J.-W. Kim, D. Lee, H. C. Shum, and D. A. Weitz, Colloid Surfactants for Emulsion Stabilization, Advanced Materials 20, 3239 (2008).

[107] Q. Xie, G. B. Davies, and J. Harting, Direct Assembly of Magnetic Janus Particles at a Droplet Interface, ACS Nano 11, 11232 (2017).

[108] J. de Graaf, M. Dijkstra, and R. van Roij, Triangular tessellation scheme for the adsorption free energy at the liquid-liquid interface: Towards nonconvex patterned colloids, Phys. Rev. E 80, 51405 (2009).

[109] J. de Graaf, M. Dijkstra, and R. van Roij, Adsorption trajectories and free-energy separatrices for colloidal particles in contact with a liquid-liquid interface, The Journal of Chemical Physics 132, 164902 (2010). 
[110] A. R. Morgan, N. Ballard, L. A. Rochford, G. Nurumbetov, T. S. Skelhon, and S. A. F. Bon, Understanding the multiple orientations of isolated superellipsoidal hematite particles at the oilwater interface, Soft Matter 9, 487 (2013).

[111] W. van der Stam, A. P. Gantapara, Q. A. Akkerman, G. Soligno, J. D. Meeldijk, R. van Roij, M. Dijkstra, and C. de Mello Donega, Self-Assembly of Colloidal Hexagonal Bipyramid- and Bifrustum-Shaped ZnS Nanocrystals into Two-Dimensional Superstructures, Nano Letters 14, 1032 (2014).

[112] B. Peng, G. Soligno, M. Kamp, B. de Nijs, J. de Graaf, M. Dijkstra, R. van Roij, A. van Blaaderen, and A. Imhof, Site-specific growth of polymers on silica rods, Soft Matter 10, 9644 (2014).

[113] N. Ballard and S. A. F. Bon, Equilibrium orientations of non-spherical and chemically anisotropic particles at liquid-liquid interfaces and the effect on emulsion stability, Journal of Colloid and Interface Science 448, 533 (2015).

[114] K. A. Brakke, The Surface Evolver, Experimental Mathematics 1, 141 (1992).

[115] M. Vis, J. Opdam, I. S. J. van 't Oor, G. Soligno, R. van Roij, R. H. Tromp, and B. H. Erné, Water-in-Water Emulsions Stabilized by Nanoplates, ACS Macro Letters 4, 965 (2015).

[116] I. Dević, G. Soligno, M. Dijkstra, R. van Roij, X. Zhang, and D. Lohse, Sessile Nanodroplets on Elliptical Patches of Enhanced Lyophilicity, Langmuir 33, 2744 (2017).

[117] M. Kamp, G. Soligno, F. Hagemans, B. Peng, A. Imhof, R. van Roij, and A. van Blaaderen, Regiospecific Nucleation and Growth of Silane Coupling Agent Droplets onto Colloidal Particles, The Journal of Physical Chemistry C 121, 19989 (2017).

[118] T. G. Anjali and M. G. Basavaraj, Contact angle and detachment energy of shape anisotropic particles at fluid-fluid interfaces, Journal of Colloid and Interface Science 478, 63 (2016).

[119] E. M. Furst, Directing colloidal assembly at fluid interfaces, Proceedings of the National Academy of Sciences 108, 20853 (2011).

[120] R. McGorty, J. Fung, D. Kaz, and V. N. Manoharan, Colloidal self-assembly at an interface, Materials Today 13, 34 (2010).

[121] A. Böker, J. He, T. Emrick, and T. P. Russell, Self-assembly of nanoparticles at interfaces, Soft Matter 3, 1231 (2007).

[122] Q. Xie, G. B. Davies, F. Günther, and J. Harting, Tunable dipolar capillary deformations for magnetic Janus particles at fluid-fluid interfaces, Soft Matter 11, 3581 (2015).

[123] M. Grzelczak, J. Vermant, E. M. Furst, and L. M. Liz-Marzán, Directed self-assembly of nanoparticles, ACS Nano 4, 3591 (2010).

[124] P. M. Chaikin and T. C. Lubensky, Principles of Condensed Matter Physics, Cambridge University Press, 1995.

[125] W. H. Evers, B. Goris, S. Bals, M. Casavola, J. de Graaf, R. van Roij, M. Dijkstra, and D. Vanmaekelbergh, Low-Dimensional Semiconductor Superlattices Formed by Geometric Control over Nanocrystal Attachment, Nano Letters 13, 2317 (2013).

[126] M. P. Boneschanscher et al., Long-range orientation and atomic attachment of nanocrystals in 2D honeycomb superlattices, Science 344, 1377 (2014). 
[127] J. J. Geuchies et al., In situ study of the formation mechanism of two-dimensional superlattices from PbSe nanocrystals, Nature Materials 15, 1248 (2016).

[128] J. G. Donaldson and S. S. Kantorovich, Directional self-assembly of permanently magnetised nanocubes in quasi two dimensional layers, Nanoscale 7, 3217 (2015).

[129] J. G. Donaldson, P. Linse, and S. S. Kantorovich, How cube-like must magnetic nanoparticles be to modify their self-assembly?, Nanoscale 9, 6448 (2017).

[130] P. Linse, Quasi-2d fluids of dipolar superballs in an external field, Soft Matter 11, 3900 (2015).

[131] L. Rossi, J. G. Donaldson, J. M. Meijer, A. V. Petukhov, D. Kleckner, S. S. Kantorovich, W. T. Irvine, A. P. Philipse, and S. Sacanna, Self-organization in dipolar cube fluids constrained by competing anisotropies, Soft Matter 14, 1080 (2018).

[132] L. Abelmann, T. A. G. Hageman, P. A. Löthman, M. Mastrangeli, and M. C. Elwenspoek, Three-dimensional self-assembly using dipolar interaction, Science Advances 6 (2020).

[133] H. R. Vutukuri, F. Smallenburg, S. Badaire, A. Imhof, M. Dijkstra, and A. Van Blaaderen, An experimental and simulation study on the self-assembly of colloidal cubes in external electric fields, Soft Matter 10, 9110 (2014).

[134] Z. Parsouzi et al., Fluctuation modes of a twist-bend nematic liquid crystal, Phys. Rev. X 6 , 21041 (2016).

[135] L. Longa and G. Pająk, Modulated nematic structures induced by chirality and steric polarization, Phys. Rev. E 93, 40701 (2016).

[136] L. Longa and W. Tomczyk, Twist-bend nematic phase in the presence of molecular chirality, Liquid Crystals 45, 2074 (2018).

[137] G. Pająk, L. Longa, and A. Chrzanowska, Nematic twist-bend phase in an external field, Proceedings of the National Academy of Sciences of the United States of America 115, E10303 (2018).

[138] M. Čopič and A. Mertelj, Q-tensor model of twist-bend and splay nematic phases, Phys. Rev. E 101, 022704 (2020).

[139] C. Greco, G. R. Luckhurst, and A. Ferrarini, Molecular geometry, twist-bend nematic phase and unconventional elasticity: a generalised Maier-Saupe theory, Soft Matter 10, 9318 (2014).

[140] A. Ferrarini, The twist-bend nematic phase: molecular insights from a generalised maier-saupe theory, Liquid Crystals 44, 45 (2017).

[141] W. Tomczyk, G. Pająk, and L. Longa, Twist-bend nematic phases of bent-shaped biaxial molecules, Soft Matter 12, 7445 (2016).

[142] A. Matsuyama, Director-pitch coupling-induced twist-bend nematic phase, Journal of the Physical Society of Japan 85, 114606 (2016).

[143] A. Matsuyama, Twist-bend nematic phases in binary mixtures of banana-shaped liquid crystalline molecules, Liquid Crystals 45, 607 (2018).

[144] A. Matsuyama, Cholesteric-isotropic phase transitions of banana-shaped liquid crystalline molecules, Molecular Crystals and Liquid Crystals 683, 3 (2019). 
[145] M. A. Osipov and G. Pająk, Polar interactions between bent-core molecules as a stabilising factor for inhomogeneous nematic phases with spontaneous bend deformations, Liquid Crystals 44, 58 (2017).

[146] M. Cestari and A. Ferrarini, Curvature elasticity of nematic liquid crystals: simply a matter of molecular shape? Insights from atomistic modeling, Soft Matter 5, 3879 (2009).

[147] M. Cestari, E. Frezza, A. Ferrarini, and G. R. Luckhurst, Crucial role of molecular curvature for the bend elastic and flexoelectric properties of liquid crystals: mesogenic dimers as a case study, J. Mater. Chem. 21, 12303 (2011).

[148] C. Greco, A. Marini, E. Frezza, and A. Ferrarini, From the molecular structure to spectroscopic and material properties: computational investigation of a bent-core nematic liquid crystal, ChemPhysChem 15, 1336 (2014).

[149] P. De Gregorio, E. Frezza, C. Greco, and A. Ferrarini, Density functional theory of nematic elasticity: softening from the polar order, Soft Matter 12, 5188 (2016).

[150] A. Mertelj, L. Cmok, N. Sebastián, R. J. Mandle, R. R. Parker, A. C. Whitwood, J. W. Goodby, and M. Čopič, Splay nematic phase, Phys. Rev. X 8, 41025 (2018).

[151] N. Chaturvedi and R. D. Kamien, Mechanisms to splay-bend nematic phases, Phys. Rev. E 100, 22704 (2019).

[152] E. F. Gramsbergen, L. Longa, and W. H. de Jeu, Landau theory of the nematic-isotropic phase transition, Physics Reports 135, 195 (1986).

[153] L. Longa, D. Monselesan, and H.-R. Trebin, An extension of the Landau-Ginzburg-de Gennes theory for liquid crystals, Liquid Crystals 2, 769 (1987).

[154] G. J. Vroege and H. N. W. Lekkerkerker, Phase transitions in lyotropic colloidal and polymer liquid crystals, Reports on Progress in Physics 55, 1241 (1992).

[155] R. Wittkowski, H. Löwen, and H. R. Brand, Derivation of a three-dimensional phase-field-crystal model for liquid crystals from density functional theory, Phys. Rev. E 82, 31708 (2010).

[156] J. C. Tolédano and P. Tolédano, The Landau Theory of Phase Transitions, WORLD SCIENTIFIC, 1987.

[157] R. van Roij, The isotropic and nematic liquid crystal phase of colloidal rods, European Journal of Physics 26, S57 (2005).

[158] R. F. Kayser and H. J. Raveché, Bifurcation in Onsager's model of the isotropic-nematic transition, Phys. Rev. A 17, 2067 (1978).

[159] D. Chen et al., Twist-bend heliconical chiral nematic liquid crystal phase of an achiral rigid bent-core mesogen, Phys. Rev. E 89, 022506 (2014).

[160] R. J. Mandle, E. J. Davis, S. A. Lobato, C.-C. A. Vol, S. J. Cowling, and J. W. Goodby, Synthesis and characterisation of an unsymmetrical, ether-linked, fluorinated bimesogen exhibiting a new polymorphism containing the ntb or "twist-bend" phase, Phys. Chem. Chem. Phys. 16, 6907 (2014).

[161] V. P. Panov, M. Nagaraj, J. K. Vij, Y. P. Panarin, A. Kohlmeier, M. G. Tamba, R. A. Lewis, and G. H. Mehl, Spontaneous periodic deformations in nonchiral planar-aligned bimesogens with a nematic-nematic transition and a negative elastic constant, Phys. Rev. Lett. 105, 167801 (2010). 


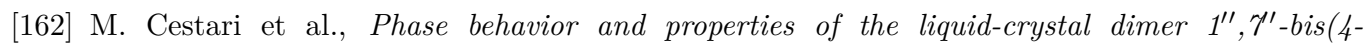
cyanobiphenyl-4'-yl) heptane: A twist-bend nematic liquid crystal, Phys. Rev. E 84, 031704 (2011).

[163] V. Borshch et al., Nematic twist-bend phase with nanoscale modulation of molecular orientation, Nature Communications 4, 2635 (2013).

[164] R. A. Reddy and C. Tschierske, Bent-core liquid crystals: polar order, superstructural chirality and spontaneous desymmetrisation in soft matter systems, J. Mater. Chem. 16, 907 (2006).

[165] R. J. Mandle and J. W. Goodby, Intercalated soft-crystalline mesophase exhibited by an unsymmetrical twist-bend nematogen, CrystEngComm 18, 8794 (2016).

[166] M. Chiappini and M. Dijkstra, A novel density-modulated twist-splay-bend phase of bananashaped particles (unpublished).

[167] L. Longa and W. Tomczyk, Twist-Bend Nematic Phase from the Landau-de Gennes Perspective, The Journal of Physical Chemistry C 124, 22761 (2020).

[168] P. G. D. Gennes, Polymeric liquid crystals: Frank elasticity and light scattering, Molecular Crystals and Liquid Crystals 34, 177 (1976).

[169] V. G. Taratuta and R. B. Meyer, Elasticity of a nematic polymer in the infinite molecular chain limit, Liquid Crystals 2, 373 (1987). 


\section{Summary}

Colloids are particles with dimensions ranging from a few nanometers up to several microns. When suspended in a solvent, colloids perform a random motion, known as Brownian motion, as a result of constant collisions with the (smaller) solvent particles. Because of the Brownian motion, colloids satisfy the so-called ergodic hypothesis, since they are in principle able to explore all possible configurations in which the system (colloids+solvent) can be. As a consequence, colloidal systems are well suited to be theoretically studied by means of equilibrium statistical mechanics. Moreover, because of their size and slow dynamics, colloids are well suited to be experimentally studied in real space and real time using advanced microscopy techniques. In this thesis we focus on two main colloidal systems: colloids trapped at a fluid-fluid interface and liquid crystalline colloidal systems.

The first part of the thesis (Chapters 2 and 3) deals with colloids at a fluid-fluid interface. Colloidal particles strongly adsorb at fluid-fluid interfaces in order to reduce the interfacial energy cost. Once adsorbed they can generate deformations in the fluid-fluid interface, which depend on the particle shape and surface chemistry. Such deformations in turn induce capillary interactions between the adsorbed particles, that can be exploited to regulate the self-assembly of the particles while confined to the fluid-fluid interface. In Chapter 2, we consider a variety of Janus particles, i. e. particles having two "faces" with different chemical surface properties, which includes dumbbells, elongated dumbbells and spherocylinders. By using a numerical method that takes into account the interfacial deformations, we numerically investigate the equilibrium configurations of these particles when adsorbed at a fluid-fluid interface, and the resulting induced interfacial deformation field. We find that the overall shape of the induced deformation field has a strong hexapolar mode while non-Janus particles of the same shape do not induce any interfacial deformations. Furthermore, we find that the hexapolar deformation field induces capillary attractions between laterally aligned Janus particles and repulsions between laterally anti-aligned ones. Our numerical predictions qualitatively explain the stabilization of aggregates of laterally aligned colloidal Janus dumbbells, recently experimentally observed at a water-decane interface. In Chapter 3, instead, we numerically study the adsorption and self-assembly of cubic colloids at a fluid-fluid interface, as a function of the wettability of the cubes. This last is quantified by the so-called Young contact angle $\theta$. We find that, while cubes with a large $\theta \sim \pi / 2$ give rise to $2 \mathrm{D}$ hexagonal structures, cubes with a smaller $\theta$ assemble into chainlike aggregates. In the hexagonal structures, the cubes are adsorbed with one vertex pointing upward with respect to the interface plane, and induce a hexapolar deformation field in the fluid-fluid interface. By contrast, cubes in the chainlike structures are adsorbed with one edge pointing upward with respect to the interface plane, and induce a quadrupolar deformation field in the fluid-fluid interface. Furthermore, for very small values of the contact angle $\theta \sim 0$, we observe square patterns of cubes adsorbed with one face pointing upward with respect to the interface plane, whose stabilization is driven by packing entropy as the capillary interactions become very weak. Our results are in agreement with the findings of recent experiments performed on $\mu \mathrm{m}$-sized polystyrene cubes. In particular our results give strong theoretical support to the idea of a capillary-driven stabilization of chains of cubic colloids at fluid-fluid interfaces, in addition to the stabilization of other, already found, 2D structures.

In the second part of the thesis (Chapters 4 and 5) we focus on lyotropic colloidal suspensions of curved or bent rods. In Chapter 4, we develop a phenomenological Landau-de Gennes 
(LdG) theory able to describe these systems, by using a $Q$-tensor expansion of the chemicalpotential dependent grand potential and introducing a bend flexoelectric term which couples the polarization and the divergence of the $Q$-tensor. We employ our theory to investigate the stability of the so-called twist-bend $\left(N_{\mathrm{TB}}\right)$ and splay-bend $\left(N_{\mathrm{SB}}\right)$ nematic phases, the former being a nematic phase characterized by a heliconical variation with bend and twist deformations in the molecular orientation, the latter being a nematic phase characterized by alternating domains of splay and bend. We also include in our picture the uniaxial nematic $(N)$ phase. Our principal findings are the following. After showing that our LdG can be completely mapped onto existing Oseen-Frank theories, we show that by removing the degeneracy between the splay and bend elastic constants, our theory can predict an $N-N_{\mathrm{TB}}-N_{\mathrm{SB}}$ phase sequence as well as an $N-N_{\mathrm{SB}}-N_{\mathrm{TB}}$ one, by increasing the particle concentration. We then focus on the first-order $N$ $N_{\mathrm{TB}}$ phase transition recently found in simulations. We find that the bend elastic constants $K_{33}$ as well as its renormalized version $K_{33}^{\text {eff }}$ remain positive at the transition, whereas $K_{33}^{\text {eff }}$ vanishes at the nematic spinodal. Finally, in Chapter 5, we consider an extension of the theory introduced in Chapter 4 that enables us to investigate the nature of the splay-bend nematic phase. In particular we consider the possibility of a spatial modulation of the nematic order parameter $S$. Since we find that in the splay-bend phase, the spatial modulations in $S$ are accompanied by a one-dimensional density modulation, we argue that the splay-bend $N_{\text {SB }}$ phase has actually the key characteristics of a smectic rather than a nematic phase. By contrast, we find that the twist-bend $N_{\mathrm{TB}}$ phase has a homogeneous density and is hence a proper nematic phase. Our results confirm recent simulation findings. 


\section{Samenvatting}

Collö̈den zijn deeltjes met een grootte van een paar nanometer tot aan meerdere microns. In een suspensie van deze deeltjes in een oplosmiddel bewegen de deeltjes met een willekeurige beweging genaamd de Brownse beweging, ten gevolge van continue botsingen met de (kleinere) deeltjes van het oplosmiddel. Dankzij deze Brownse beweging voldoen colloïden aan de zogenoemde ergodische hypothese, aangezien ze in principe alle mogelijke configuraties van het systeem (colloïden+oplosmiddel) kunnen verkennen. Dankzij deze eigenschap zijn collö̈dale systemen zeer goed theoretisch te beschijven met de evenwichts statistische mechanica. Bovendien zijn ze dankzij hun grootte en langzame dynamica erg geschikt om experimenteel te bestuderen in de reële ruimte en tijd met behulp van geavanceerde microscopietechnieken. In dit proefschrift richten we ons hoofdzakelijk op twee collö̈dale systemen: colloïden gevangen op het grensvlak tussen twee vloeistoffen, en colloïdale vloeibaar kristallen.

Het eerste deel van dit proefschrift (Hoofdstuk 2 en 3) gaan over colloïden op een vloeistofvloeistofgrensvlak. Collö̈dale deeltjes adsorberen sterk aan een vloeistof-vloeistofgrensvlak omdat ze daarmee de grensvlakenergie verminderen. Eenmaal geadsorbeerd kunnen ze vervormingen teweegbrengen in het vloeistof-vloeistofgrensvlak die afhangen van de vorm van het deeltje en de chemische eigenschappen van diens oppervlak. Zulke vervormingen veroorzaken vervolgens capillaire interacties tussen de geadsorbeerde deeltjes die kunnen worden gebruikt om hun zelf-assemblage in het grensvlak aan te sturen. In Hoofdstuk 2 beschouwen we een verzameling aan Janusdeeltjes - deeltjes met twee "gezichten" die verschillende chemische oppervlakteeigenschappen hebben - met onder andere haltervormige, langwerpig haltervormige en spherocylindrische deeltjes. Door gebruik te maken van een numerieke methode die de vervormingen van het grensvlak meerekenen, bestuderen we numeriek de evenwichtsconfiguraties van deze deeltjes geadsorbeerd aan een vloeistof-vloeistofgrensvlak en het resulterende grensvlakvervormingsveld. We vinden dat de algemene vorm van het geinduceerde vervormingsveld een sterk hexapolaire modus heeft terwijl niet-Janusdeeltjes met dezelfde vorm geen grensvlakvervorming veroorzaken. Verder vinden we dat het hexapolaire vervormingsveld capillaire attracties veroorzaakt tussen lateraal opgelijnde en repulsies tussen lateraal anti-opgelijnde Janusdeeltjes. Onze numerieke voorspellingen beschrijven kwalitatief de stabilizatie van aggregaten van lateraal opgelijnde colloïdale Janushalters die recentelijk experimenteel zijn waargenomen op een water-decaangrensvlak. In Hoofdstuk 3 bestuderen we numeriek de adsorptie en zelfassemblage van kubische collö̈den op een vloeistof-vloeistofgrensvlak als een functie van hun bevochtigbaarheid. Dit laatste wordt gekwantificeerd met de zogenoemde Young contacthoek $\theta$. We vinden dat hoewel kubussen met een grote $\theta \sim \pi / 2$ leidden tot $2 \mathrm{D}$ hexagonale structuren, kubussen met een kleinere $\theta$ juist ketenvormige aggregaten vormen. In de hexagonale structuren zijn de kubussen geadsorbeerd met een vertex omhoog uit het oppervlak gericht, en induceren ze een hexapolair vervormingsveld in het vloeistof-vloeistofgrensvlak. Daarintegen zijn kubussen in de ketenvormige structuren geadsorbeerd met een ribbe omhoog gericht ten opzichte van het oppervlak, en induceren ze een quadrupolair vervormingsveld in het vloeistofvloeistofgrensvlak. Verder observeren we voor zeer kleine waardes van de contacthoek $\theta \sim 0$ vierkante patronen van kubussen geadsorbeerd met een vlak omhoog gericht ten opzichte van het oppervlak, wiens stabilisatie veroorzaakt wordt door de pakkingsentropie wanneer de capillaire interacties zeer zwak worden. Onze resultaten zijn in overeenstemming met de bevindingen van recente experimenten gedaan aan $\mu \mathrm{m}$-grootte polystyreenkubussen. Met name geven onze 
resultaten een sterke theoretische steun aan het idee van een capillair-gedreven stabilisatie van ketens van kubische colloïden aan een vloeistof-vloeistofgrensvlak, bovenop de stabilisatie van andere al eerder gevonden 2D structuren.

In het tweede deel van dit proefschrift (Hoofdstuk 4 en 5) richten we ons op lyotrope colloïdale suspensies van gebogen of geknakte staven. In Hoofdstuk 4 ontwikkelen we een fenomenologische Landau-de Gennes (LdG) theorie om deze systemen te beschrijven door middel van een $Q$-tensor expansie van de chemischpotentiaalafhankelijke groot-potentiaal en de introductie van een buigingsflexo-electrische term die de polarizatie en divergentie van de $Q$-tensor koppelt. We gebruiken onze theorie om te stabiliteit van de zogeheten twist-buiging $\left(N_{\mathrm{TB}}\right)$ en splay-buiging $\left(N_{\mathrm{SB}}\right)$ nematische fasen te bestuderen. De eerstgenoemde is een nematische fase die gekarakteriseerd wordt door een helische variatie van de buigings- en twistvervormingen in de moleculaire orientaties, en de laatstgenoemde een nematische fase gekarakteriseerd door afwisselende domeinen van splay en buiging. Ook beschouwen we de eenassige nematische $(N)$ fase. Onze belangrijkste bevindingen zijn als volgt. Nadat we eerst laten zien dat onze LdG theorie volledig kan worden verbonden aan bestaande Oseen-Frank theorieën, tonen we vervolgens aan dat door de degeneratie tussen de splay en buiging elastische constanten te verwijderen onze theorie een $N-N_{\mathrm{TB}}-N_{\mathrm{SB}}$ en een $N-N_{\mathrm{SB}}-N_{\mathrm{TB}}$ fasereeks voorspelt voor een toenemende deeltjesconcentratie. Hierna richten we ons op de eerste-orde $N-N_{\mathrm{TB}}$ faseovergang die recent gevonden is in simulaties. We vinden dat de buigings elastische constante $K_{33}$ en diens gerenormaliseerde versie $K_{33}^{\text {eff }}$ positief blijven bij de overgang, terwijl $K_{33}^{\text {eff }}$ verdwijnt bij de nematische spinodaal. Tot slot beschouwen we in Hoofdstuk 5 een uitbreiding van de de theorie ontwikkeld in Hoofdstuk 4 die het mogelijk maakt om de ware aard van de splay-buiging nematische fase te onderzoeken. We beschouwen met name de mogelijkheid van een ruimtelijke modulatie van de nematische ordeparameter $S$. Omdat we in de splay-buiging fase vinden dat ruimtelijke modulaties van $S$ samengaan met een eendimensionale dichtheidsmodulatie beargumenteren we dat de splay-buiging $N_{\mathrm{SB}}$ fase eigenlijk een smectische fase is in plaats van een nematische. De twist-buiging $N_{\mathrm{TB}}$ fase heeft daarintegen een homogene dichtheid en is dus een daarwerkelijk een nematische fase. Onze resultaten bevestigen recente simulatiebevindingen. 


\section{Acknowledgements}

First of all my deepest gratitude goes to my supervisors Marjolein Dijkstra and René van Roij for accepting me as a $\mathrm{PhD}$ candidate in their group. Dear Marjolein and René, working with you has been a true honor for me, something I will be always proud of. I have literally learned how to be a scientist from you. Besides that, you have always been available for stimulating discussions and impeccable revision of papers, slides and thesis chapters (even in the last "strange" times!). Thank you so much for everything!

Then I would like to thank Laura Filion, from which I have learned a lot when I was teaching assistant of her Advanced Statistical Physics course, and Joost de Graaf (from the Institute for Theoretical Physics) for several fruitful discussions. Many thanks to the "experimental" staff of the Soft Condensed Matter Group (SCM): Alfons van Blaaderen, Arnout Imhof, Patrick Baesjou, Krassimir Velikov, Marijn van Huis and Freddy Rabouw. Their valuable comments and suggestions have really helped me in improving the quality of my research. Thanks a lot also to Hester van der Putte, Dianne Ickenroth and Marion Wijburg for their kind help with administrative tasks and to Peter Helfferich for his help with technical difficulties.

I am deeply grateful to my master's thesis supervisor Giorgio Pastore for introducing me to the soft matter physics and referring me to the Utrecht group.

Half of the work presented in this thesis would have not been possible without the help of Giuseppe Soligno. Thanks a lot, Giuseppe, for sharing with me your (amazing) numerical code in the initial stage of my $\mathrm{PhD}$, for the very nice collaborations on the "dumbbells and cubes at interfaces", and for the infinite number of discussions we had in the past years! One of our collaborations involved also Fuqiang Chang who performed the experimental work in the group of Willem K. Kegel. Dear Fuqiang and Willem, it has been a true pleasure to collaborate with you.

I would like to thank Simone Dussi, one of the most talented colleague I have met so far. Thank you very much, Simone, for introducing me to the physics of liquid crystals and for the (failed) collaborations. A very big thank you goes to Giulia Fiorucci and Guido Avvisati. Without your help guys (inside and outside SCM) it would have been impossible for me to finalize this thesis. Dear Siddharth Paliwal, thank you for your constant kindness and help. You are one of the best people I met in this journey!

During my PhD time I have been very fortunate to share the office with Naveed Hosseini. Dear Naveed, I should write another thesis to properly thank you for all the help you gave me in the past years! Also there is not enough space here to properly thank Rama Kotni. Guys, I have seen you working so hard in the last years. I wish you a future of great achievements!

My PhD time was also very nice since I had the opportunity to meet and spend time with Douglas Hayden, Tonnishtha Dasgupta, Chris Kennedy, Anna Nikolaenkova, Jessi van der Hoeven and Pepijn Moerman. Thank you very much, guys, for the nice moments inside and outside SCM!

I also sincerely thank all the other colleagues that I have met in the SCM group. In particular I acknowledge the "senior" colleagues that introduced me to the group: Ernest van der Wee, Wessel Vlug, Vassilis Prymidis, Fabian Hagemans and Srivatssan Mohan, as well as the colleagues that started their PhD (more or less) at the same time as me: Robin van Damme, Albert Grau, Tom Welling and Sina Sadighikia. A special thank goes to Da Wang for all the weekends we spent together in the OL! To Alberto Orellana, instead, a big good luck for his 
$\operatorname{PhD}$.

Robin van Damme and Douglas Hayden are also thanked for their kind help in translating the summary of this thesis from english to dutch.

I also would like to thank some colleagues from the Institute for Theoretical Physics. Thanks to Ben Werkhoven for introducing me to COMSOL Multiphyiscs, and to Tara Drwenski for interesting discussions on liquid crystals.

Desidero inoltre ringraziare il mio amico Davide per l'aiuto nella realizzazione della cover di questa tesi. Grazie di cuore anche a Sabrina per avermi aiutato in un momento complicato. Dedico infine questa tesi alla mia famiglia. 


\section{List of publications}

This thesis is based on the following publications:

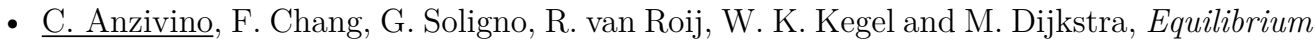
configurations and capillary interactions of Janus dumbbells and spherocylinders at fluidfluid interfaces, Soft Matter 15, 2638 (2019) (Chapter-2)

- C.Anzivino, G. Soligno, R. van Roij and M. Dijkstra, Chains of cubic colloids at fluid-fluid interfaces, Soft Matter 17, 965 (2021) (Chapter-3)

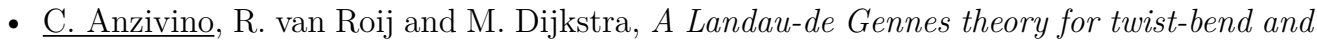
splay-bend nematic phases of colloidal suspensions of bent rods, The Journal of Chemical Physics 152, 224502 (2020) (Chapter-4)

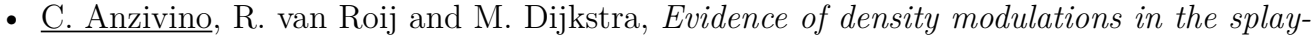
bend nematic phase of colloidal suspensions of bent rods, manuscript in preparation (Chapter-5) 


\section{Oral and poster presentations}

The content of this thesis was also presented at the following events:

- Physics@FOM, January 23-24 2018, Veldhoven (The Netherlands) poster: Self-assembly of dumbbells at a fluid-fluid interface authors: C. Anzivino, G. Soligno, R. van Roij and M. Dijkstra

- Physics@FOM, January 21-22 2019, Veldhoven (The Netherlands) poster: A Landau-de Gennes theory for lyotropic liquid crystals authors: C. Anzivino, R. van Roij and M. Dijkstra

- Physics@FOM, January 21-22 2020, Veldhoven (The Netherlands) oral talk: Equilibrium configurations and capillary interactions of Janus particles at a fluid-fluid interface

authors: C. Anzivino, F. Chang, G. Soligno, R. van Roij, W. K. Kegel and M. Dijkstra 


\section{About the author}

Carmine Anzivino was born on the $11^{\text {th }}$ December 1990 in the city of Foggia, in Italy. After completing high school in Foggia (Liceo Scientifico "A. Volta") in 2009, he moved to Bari. In this city he received his Bachelor's degree in Physics in 2013. He then moved to Trieste where he received his Master's degree cum laude in Theoretical Physics in 2016. The thesis project was entitled "Integral equations and spontaneously broken replica symmetry in the liquid-glass transition" and was supervised by Prof. Giorgio Pastore. From the $1^{\text {st }}$ July 2016 he has been a $\mathrm{PhD}$ student in the Soft Condensed Matter group of the Physics department at Utrecht University under the supervision of Prof. Marjolein Dijkstra and Prof. René van Roij. His research focused on the theoretical description of colloidal suspensions and is exposed in this thesis. He presented his work to the scientific community in both national and international conferences, while publishing his results in peer-reviewed journals. 


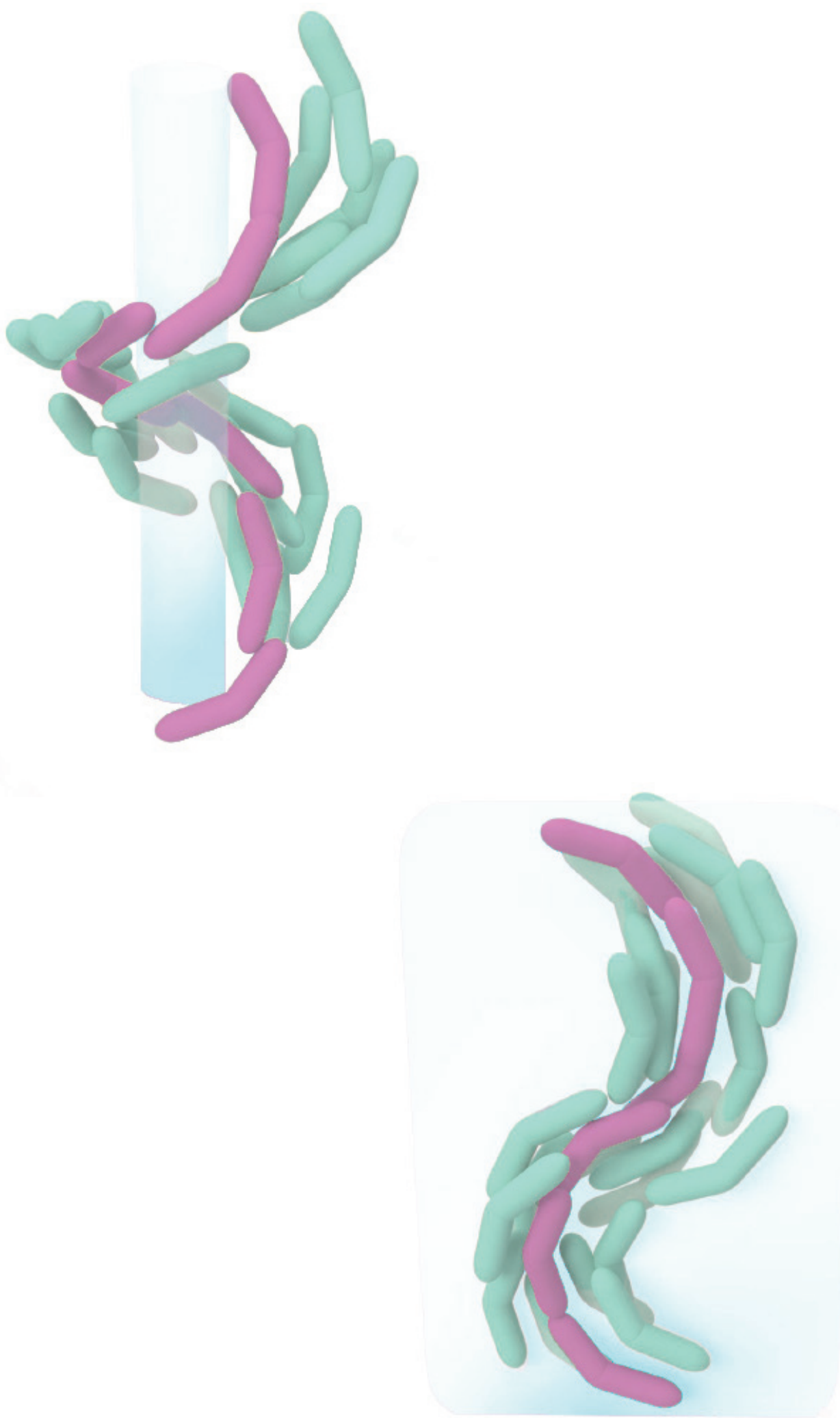